\title{
NONLINEAR DYNAMICS OF NETWORKS: THE GROUPOID FORMALISM
}

\author{
MARTIN GOLUBITSKY AND IAN STEWART
}

\begin{abstract}
A formal theory of symmetries of networks of coupled dynamical systems, stated in terms of the group of permutations of the nodes that preserve the network topology, has existed for some time. Global network symmetries impose strong constraints on the corresponding dynamical systems, which affect equilibria, periodic states, heteroclinic cycles, and even chaotic states. In particular, the symmetries of the network can lead to synchrony, phase relations, resonances, and synchronous or cycling chaos.

Symmetry is a rather restrictive assumption, and a general theory of networks should be more flexible. A recent generalization of the group-theoretic notion of symmetry replaces global symmetries by bijections between certain subsets of the directed edges of the network, the 'input sets'. Now the symmetry group becomes a groupoid, which is an algebraic structure that resembles a group, except that the product of two elements may not be defined. The groupoid formalism makes it possible to extend group-theoretic methods to more general networks, and in particular it leads to a complete classification of 'robust' patterns of synchrony in terms of the combinatorial structure of the network.

Many phenomena that would be nongeneric in an arbitrary dynamical system can become generic when constrained by a particular network topology. A network of dynamical systems is not just a dynamical system with a high-dimensional phase space. It is also equipped with a canonical set of observables - the states of the individual nodes of the network. Moreover, the form of the underlying ODE is constrained by the network topology - which variables occur in which component equations, and how those equations relate to each other. The result is a rich and new range of phenomena, only a few of which are yet properly understood.
\end{abstract}

\section{Contents}

1. Introduction

2. Symmetry and synchrony

3. Animal locomotion

4. Is symmetry necessary for synchrony?

5. Coupled cell networks

6. Admissible vector fields

7. Balanced equivalence relations

Received by the editors May 2, 2005.

2000 Mathematics Subject Classification. Primary 37G40, 34C23, 34C25, 92B99, 37G35.

Part of this material was presented by M. Golubitsky in the SIAM plenary lecture "Coupled cell systems: A potpourri of theory and examples", given at the Joint Mathematics Meetings in Phoenix, AZ, January 2004.

(C)2006 American Mathematical Society Reverts to public domain 28 years from publication 
8. Rigid equilibria

9. Quotient networks

10. Rigid periodic states

11. Synchrony-breaking bifurcations

12. Interior symmetries

13. Phase equations

14. Synchronized chaos

15. Bubbling bifurcation

16. Is there a linear theory?

Acknowledgments

About the authors

References

\begin{abstract}
There are plenty of objects which exhibit what we clearly recognize as symmetry, but which admit few or no nontrivial automorphisms. It turns out that the symmetry, and hence much of the structure, of such objects can be characterized algebraically, if we use groupoids and not just groups.
\end{abstract}

Alan Weinstein 83

\title{
1. INTRODUCTION
}

The biologist J.B.S. Haldane, when asked what we can learn about the Creator by examining the world, replied that God seemed to have an inordinate fondness for beetles. Today's biologists could be forgiven for pointing to the deity's inordinate fondness for networks. Networks are ubiquitous in biology: examples include gene expression, neural circuitry, ecological food webs, and disease transmission. Networks are also common in many other branches of science, and there has been a recent explosion of interest in the topic. The research literature, including applications, now extends to many thousands of papers.

Mathematically, a network is a directed graph whose nodes represent state variables and whose directed edges represent interactions among those variables. In this paper we usually assume that the graph is finite, but most of the ideas extend to locally finite graphs. What interests us here is processes taking place on networks. The nodes and edges are equipped with some kind of 'dynamic', which could be a choice of moves (games), a transition probability (Markov chains), discrete states in time and space (cellular automata), or continuous states (coupled ODEs, the subject of the present discussion). Analogies of the groupoid formalism that we introduce below can be developed for any sensible type of process on a network, but this area is virtually unexplored as we write.

Much progress has been made on how combinatorial features of networks affect their static or statistical behavior. The work of Watts and Strogatz 82 on 'small world' networks is especially well known. For surveys of the field, see Boccaletti et al. 10] or Wang [80. Probabilistic aspects of networks have also received a lot of attention, Newman 63. However, general studies of network dynamics are fairly rare: of the 429 references cited by Newman, only 6 are about dynamics (though, admittedly, dynamics is not the main target of that paper). Many papers on network dynamics make restrictive assumptions, a typical one being to assume weak linear coupling, as in Kuramoto [54 and Ashwin and Swift [7. Wu [90] 
uses a Liapunov function to derive conditions for stable synchronization of systems with identical internal dynamics, assuming linear (time-dependent) coupling. Feinberg [26] and Tyson et al. [77, 78] study cell systems where the dynamical equations are chemical reaction equations. Feinberg has beautiful results about when the network can support steady states and periodic solutions; Tyson et al. have derived detailed cell cycle models along with an understanding of how the network affects the dynamics in these models. Most of the rest are about numerical simulations of specific models. There are several excellent books, including Manrubia et al. [57, Mosekilde et al. 60], and Wu [89. All three of these discuss applications.

Here we describe a formal framework for the nonlinear dynamics of networks, based on the recent discovery of an algebraic context for such questions, namely the symmetry groupoid of the network [72. This is a natural generalization of the concept of a symmetry group. The main difference between groupoids and groups is that in a groupoid the product of two elements may not be defined. This difference has significant knock-on effects, and the theory of groupoids has a different flavor from that of groups; see Brandt [1], Higgins [47, and Brown [12.

The symmetry group of a network (that is, the automorphism group of the graph) is known to have a strong influence on its dynamics [6, 20, 21, 25, 30, 36, 37, 40, 41, 86, 87, 88. Evidence is accumulating that many generic features of the dynamics of asymmetric networks can be understood from the groupoid viewpoint (where 'generic' is relative to the network structure). As for conventional dynamical systems, quantitative information about specific models must usually be obtained through numerical simulations, but the conceptual framework helps us to organize and understand the results of such simulations and to predict their general qualitative features.

Our framework applies to networks whose architecture is fixed, and we shall not discuss cases where the architecture itself evolves over time, even though this is a very interesting topic. It is worth observing, however, that the 'local' nature of groupoid symmetries implies that small changes in the architecture - such as the addition or removal of a node or an edge - may preserve large parts of the groupoid. Global group-theoretic symmetries are more easily destroyed by small changes in architecture. It is also worth remarking that many popular examples of networks, such as the Internet, do not fit directly into our scheme because the nodes are not naturally modeled by dynamical systems. However, the groupoid concept applies to the topology of the network, independently of the structure associated with a node, and it can readily be generalized to other mathematical contexts, for instance Markov chains.

It remains to be seen how useful the groupoid formalism will be for applied science. The group-theoretic approach to pattern-formation now has many applications, including several to networks, the latter mostly in connection with neuroscience. The groupoid formalism is most likely to be useful for networks constructed from multiple copies of a few basic 'components', both for cells and for couplings, and where the network architecture is fairly repetitive. Such networks occur in several areas of science. Generalizations of the theory that permit approximate or statistical symmetries may be feasible and, if so, may be more suitable for some applications. The phenomenon of 'motifs' - unusually common small subnetworks, Milo et al. [59], Wolf and Arkin [85, Dobrin et al. [22] -is also worth mentioning in this context. However, as we write, there exist few applications of the groupoid 
formalism as such. Because it is a natural generalization of the successful grouptheoretic formalism and because the mathematical theory is rich and challenging, we believe that the prospects for useful applications are good. But we also anticipate a period of further development before it becomes clear whether we are right. At any rate, the topic is an interesting area of pure dynamical systems theory with a fascinating interplay of techniques.

Dynamic Features Specific to Networks. It is important to appreciate that a network is not just a high-dimensional dynamical system, even though its associated ODEs can be interpreted as precisely that. The novel feature is that the nodes of the network define distinguished observables whose dynamics can be compared. Two nodes may be (exactly or approximately) synchronous, they may have related phases or frequencies, and they may behave chaotically yet still resemble each other.

To illustrate these points in an informal setting, we briefly consider the simplest nontrivial network: two identical cells with identical coupling. Schematically such a system is represented as in Figure 1, and this network has an obvious symmetry which swaps the two nodes and the two edges. Abstractly, this group is a cyclic group $\mathbf{Z}_{2}$ of order 2 .

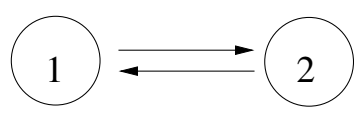

FIGURE 1. Schematic of a two-cell network with identical cells and identical coupling.

We associate with this network a class of differential equations, which we call 'admissible'. For this network the admissible differential equations are those of the form

$$
\begin{aligned}
& \dot{x}_{1}=g\left(x_{1}, x_{2}\right) \\
& \dot{x}_{2}=g\left(x_{2}, x_{1}\right)
\end{aligned}
$$

where $x_{1}, x_{2} \in \mathbf{R}^{k}$ are the state variables of the individual cells. Observe that a single function $g: \mathbf{R}^{k} \times \mathbf{R}^{k} \rightarrow \mathbf{R}^{k}$ defines the system.

One consequence of the $\mathbf{Z}_{2}$ symmetry of this system is the existence of solutions in which $x_{1}(t)=x_{2}(t)$ for all $t$. This follows because the 'diagonal' subspace $\left\{x: x_{1}=x_{2}\right\}$ is invariant under the flow of the differential equation for all $g$. For all such solutions, the two cells behave synchronously.

Another consequence of symmetry is that there exists a nonempty open set of functions $g$ for which there is a periodic solution, with period $T$, such that $x_{2}(t)=x_{1}(t+T / 2)$ for all $t$; see [41, 37. That is, the two cells have the same periodic dynamics except for a relative phase shift of half a period.

It turns out that both synchrony and phase relations occur naturally for many other networks, even when the only global (group-theoretic) symmetry is trivial. We will show that the symmetry groupoid goes a long way towards explaining both of these phenomena and can be viewed as a natural algebraic framework for such comparisons.

We say that a network exhibits synchrony if two or more cells behave (more or less) identically. Synchrony is a fundamental phenomenon in networks, and there is a huge literature, most of which we shall not cite here. In models of speciation in 
evolutionary biology (Cohen and Stewart [15, Elmhirst [23], Stewart et al. 71], Vincent and Vincent [79]) the nodes of the network represent (coarse-grained sets of) organisms, the edges represent interactions (breeding, competition for resources), and synchronized clusters correspond to species. In analogous models of pathological microorganisms, synchronized clusters correspond to genetically closely related strains (Gomes and Medley [43]). In neurobiology (Kopell and LeMasson [53]) synchronized clusters correspond to groups of nerve cells that 'fire together', which is significant for neural processing and the architecture of the brain (Singer [70]). Manrubia et al. [57] discuss synchronization in neural networks. Mosekilde et al. 60] describe a model of synchronization in nephrons, structures in the kidneys that help regulate blood pressure.

Similarly, we say that two cells are phase-related if they have identical periodic dynamics except for fixed phase shifts. Phase-relation is another important network phenomenon. Such patterns are central to animal locomotion (Kopell and Ermentrout [51, 52, Collins and Stewart [16, 17, Buono and Golubitsky [13]). Phase-locked waves of activity are observed in the leech heartbeat (Calabrese and Peterson [14], Olsen and Calabrese [64]) and elsewhere. The nephron model of Mosekilde et al. 60 also exhibits phase-related states.

As the two-cell example suggests, there is one general context in which synchrony and phase-relations are entirely natural and easily explained, namely, dynamics with symmetry. If the network is symmetric, then the constraints imposed on the dynamics by symmetry often lead to cells being synchronous or phase-related. The theory to be outlined in this paper grew from the realization that although symmetry is sufficient to make these phenomena natural, it is not necessary. Certain types of network architecture, with trivial symmetry, still imply the 'generic' existence of synchronized states and of phase-related states. This realization led to a weaker concept of 'symmetry' for dynamics on a network; this formalism in turn suggested numerous questions, some of which we can answer, while others remain mysterious.

In order to draw out the themes of this article, we list a few of the broader questions raised by the groupoid viewpoint:

- How does the combinatorics (graphs) affect the algebra (groupoids), and how does the algebra affect the dynamics?

- Which dynamic phenomena become likely because of the network constraints?

- What are the existence theorems?

- What are the linear conditions for stable equilibria, and what are the nonlinear bifurcation theorems?

- What are the analogies with the group case? When do these analogies fail and why?

Outline of the Paper. Section 2 introduces some basic ideas from symmetric dynamics and examines how symmetry leads to synchrony and phase relations. These phenomena are codified by the $H / K$ Theorem, which classifies the possible spatiotemporal symmetries of periodic states for symmetric systems. Section 3 applies the resulting techniques to patterns that arise in the motion of four-legged animals, illustrating the constraints imposed on dynamics from network architecture and demonstrating that features of the architecture can be inferred (subject to certain modeling assumptions that can be made explicit) from observed dynamics. This 
application also shows that this approach has some predictive value and can be tested.

Section 3 broadens the scope of enquiry. We present several examples of networks in which synchrony and phase relations can be observed, even though the network lacks symmetry in the group-theoretic sense. These patterns are 'robust': that is, they cannot be destroyed by small changes to the ODEs that respect the network architecture. Note that robustness is not the same as stability, which concerns small changes in initial conditions; in particular, robust states may be unstable. The (linear) stability of any given state is essentially a computational issue in linear algebra and can be worked out directly for any specific network (provided it is too large), so we mainly focus on issues of existence. Stability issues are basic to bifurcation theory, however, and are discussed in more detail in the corresponding sections of the paper.

The examples in Section 3 motivate two key ideas: that patterns of synchrony are related to 'local' symmetries of the 'input sets' of the synchronous cells and that the type of synchronous dynamic that occurs is determined by a 'quotient network' in which synchronous cells are identified. We formalize the notion of 'coupled cell network' in Section [5] and describe the associated 'admissible' ODEs in Section 6. Section 7 introduces the notion of a 'balanced' equivalence relation, which characterizes robust patterns of synchrony and leads to a precise definition of a quotient network. Proofs of these results are found in [42, 72. We apply this characterization to some patterns that arise in lattice dynamics - with a few surprises (Wang et al. [3, 81]).

Robust synchrony is a very strong requirement: it occurs because of the presence of subspaces that are flow-invariant for all admissible ODEs. Section 8 shifts the emphasis to synchronous states rather than entire subspaces. The main conclusion is that this makes no difference: the synchrony relation is still balanced. We prove this when the state concerned is a hyperbolic equilibrium [42. We conjecture that it is true for any hyperbolic attractor, but a proof is currently lacking and the issues involved are not straightforward.

Moving away from equilibria to more complex dynamics, Section 9 defines quotient networks and relates their dynamics to the original network. The key theorem is that any admissible dynamic on the quotient lifts to a synchronous dynamic on the original network 42]. Therefore we can deduce the existence of synchronized states with complex dynamics - periodic, quasiperiodic, chaotic, etc. The periodic case is studied in Section 10 and here the theory becomes more conjectural. A plausible (but as yet unproved) conjecture, the Rigid Phase Conjecture, states that for a hyperbolic periodic state, phase-related cells have phase-related input sets (with the same phase-shift). Assuming this conjecture, we can prove that rigid phase relations always arise from a quotient network with cyclic group symmetry.

The discussion to this point concerns individual states of the system, that is, possible phenomena occurring in individual solutions. The paper now moves in a new direction: bifurcation. Bifurcations occur when the dynamics of a system changes radically as a parameter is varied. Examples include steady-state bifurcation, where an equilibrium becomes unstable and new equilibria appear nearby, and Hopf bifurcation, where an equilibrium becomes unstable and gives rise to a periodic state. Bifurcation theorems are useful ways to prove the existence of certain states. 
Such bifurcations are relatively well understood in the case of symmetric systems [37, 41, and here the central phenomenon is 'symmetry-breaking': the symmetry of the state can change. The analogous concept in general networks is 'synchrony-breaking', in which a synchronized state loses stability and other states arise.

There are two main issues here. One is a linear algebra question: the conditions for a state to become unstable are determined by the eigenvalues of the linearized vector field. The other question involves nonlinear terms: to deduce what form the bifurcations take when instability sets in. We show that the network architecture has a significant (and puzzling) influence on both of these issues.

Section 111 introduces synchrony-breaking bifurcations and opens Pandora's box with a 3-cell feed-forward network studied in [24, 32. Here we find the occurrence of a Hopf bifurcation in which the first cell remains in equilibrium, the second becomes periodic with an amplitude that grows according to the square root of the bifurcation parameter, and the third becomes periodic with an amplitude that grows according to the sixth root of the bifurcation parameter. This 'anomalous' behavior is generic with such a network architecture.

With Pandora's box open and its contents released, it becomes clear that network architecture can have a major effect on generic bifurcation. Numerous phenomena that would be highly exotic, or degenerate, in a single dynamical system become typical in a network. The classification and analysis of these bifurcations is complicated by curious problems of linear and nonlinear algebra. We manage to bring a semblance of order into one tiny corner of this vast area in Section 12, which introduces a type of network symmetry that is somehow intermediate between global group symmetry and local groupoid symmetry. We call it 'interior symmetry', and we discuss analogues of the Equivariant Branching Lemma for steady-state bifurcation and the Equivariant Hopf Theorem for bifurcation to periodic states proved in 33 .

The theory derived at this stage of the paper, modulo a few plausible conjectures, is reasonably coherent and complete. However, it is restricted either to general observations about invariant subspaces or to properties of equilibria and periodic states - not terribly exciting dynamically. Moreover, the cell phase spaces are assumed to be vector spaces (an assumption motivated by local bifurcation theory), which rules out any discussion of phenomena associated with the global topology of the cell phase spaces. Sections 13 15 address these issues, although they raise more questions than they answer.

Phase spaces with nontrivial topology are important in the network literature, because it is common to model synchrony and phase relations in periodic dynamics in terms of phase oscillators, where the cell phase space is a circle; see Kopell and Ermentrout 52, and Hoppensteadt and Izhekevich [49. With this choice of cell phase space, oscillations have frequency but no specific amplitude. In Section 13 we follow 31 and apply the theory to such networks and deduce strong constraints on rotation numbers of cells in all-to-all coupled networks. We round off our discussion of synchronized states in Section 14, which discusses synchronized chaos. Section 15 develops this topic by analyzing an associated bifurcation scenario, the 'bubbling' bifurcation in synchronized chaos. Here, the chaotic state repeatedly loses and regains synchrony. This example relates our idealized form of synchrony (states are identical) to the more approximate synchrony often seen in applications. 
Finally, we provide a speculative postscript directed at a key unsolved question which is central to the understanding of the stability of equilibria, periodic states, and synchronized chaotic states. Section 16 discusses the linear algebra questions involved in determining stabilities of network states, which opens up some intriguing but puzzling problems. It seems clear that much more remains to be said.

\section{SYMMETRY AND SYNCHRONY}

Issues of synchrony and phase-relations are well understood in a more specialized context: symmetric networks. This context provides a natural entry route into the more general setting that we wish to introduce and is more accessible. At this stage we therefore formalize 'symmetry' as invariance with respect to some group of transformations. The resulting theory applies not just to symmetric networks but to symmetric dynamical systems in general.

We therefore begin by reviewing the theory of symmetry in nonlinear dynamics from the group-theoretic viewpoint, [37, 41, but with emphasis on the network point of view. These results motivate the development of the generalization to networks with groupoid 'symmetries'. The crucial difference is that group symmetries are transformations of the network that preserve its global architecture, whereas groupoid symmetries relate local regions of the network to other regions. For the moment, though, we confine the discussion to group symmetries.

A symmetry of an ordinary differential equation (ODE) is a transformation that sends solutions to solutions. More precisely, let $\gamma: \mathbf{R}^{n} \rightarrow \mathbf{R}^{n}$ be a linear map. A system of differential equations

$$
\dot{x}=f(x)
$$

(where $x \in \mathbf{R}^{n}$ and $f: \mathbf{R}^{n} \rightarrow \mathbf{R}^{n}$ is smooth) has symmetry $\gamma$ if $\gamma x(t)$ is a solution to (2.1) whenever $x(t)$ is a solution. It is straightforward to verify that $\gamma$ is a symmetry if and only if $f$ satisfies the equivariance condition

$$
f(\gamma x)=\gamma f(x) .
$$

In contrast, a symmetry of a network is a purely combinatorial concept: a transformation that preserves the network architecture. It can be defined as a pair of permutations, one of the nodes and one of the edges, that preserve incidence relations between nodes and edges. Each symmetry of a network induces a symmetry of any corresponding ODE. The set of all network symmetries is a group (its graph-theoretic automorphism group).

These two notions are related, because the ODEs that describe the dynamics of a symmetric network inherit its symmetries. This relationship is a natural consequence of the constraints imposed on the ODEs by the network architecture. We can therefore apply the theory of symmetric dynamical systems to symmetric networks. The most immediate result is that both synchrony and phase-locking are natural consequences of network symmetry [20, 21, 35, 37, 41].

Example 2.1. To provide a concrete example of dynamics in a symmetric network, we consider a specific system of ODEs determined by the two-cell network of Figure 1, based on the FitzHugh-Nagumo model of a neuron (FitzHugh [28, Nagumo et al. 61]). For a single neuron, the variables assumed in this model are a membrane potential $v$ and (a surrogate for) an ionic current $w$. The state of the 
neuron is thus specified by a point $(v, w) \in \mathbf{R}^{2}$, and the internal dynamic is

$$
\begin{aligned}
\dot{v} & =v(a-v)(v-1)-w \\
\dot{w} & =b v-\gamma w
\end{aligned}
$$

where $a, b, \gamma$ are parameters, and $0<a<1, b>0, \gamma>0$.

In a two-cell network, the internal dynamic of one cell is modified by coupling effects from the other cell. In the Fitzhugh-Nagumo model, coupling can (for instance) occur by adding an applied current to the $\dot{v}$ equation, this being a function of the state of the other cell. Thus $x_{1}=\left(v_{1}, w_{1}\right), x_{2}=\left(v_{2}, w_{2}\right)$, and

$$
g\left(x_{1}, x_{2}\right)=\left(v_{1}\left(a-v_{1}\right)\left(v_{1}-1\right)-w_{1}-c v_{2}, b v_{1}-\gamma w_{1}\right)
$$

where $c$ represents coupling strength. The origin is a stable equilibrium for the full four-dimensional system when $a=b=\gamma=0.5$ and $c=0.9$. Hence the cells are (trivially) synchronous. When $c=1.1$ the two-cell system has a stable periodic solution with the two cells one half period out of phase. See Figure 2

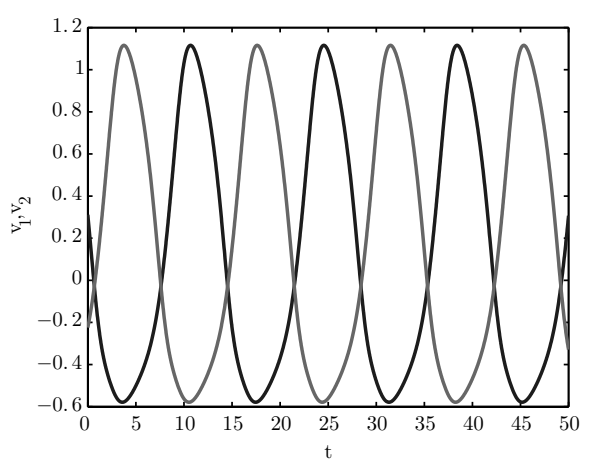

Figure 2. Two coupled Fitzhugh-Nagumo neurons exhibiting a half period out of phase periodic solution. Time series of $v_{1}, v_{2}$ in (1.1) using $g$ in (2.4).

As indicated in the introduction to this paper, these two phenomena are grouptheoretic consequences of the symmetry of the two-cell network rather than the specific choice of model. We now discuss this point in a little more detail.

The symmetry is visible in the figure and in the equations: all two-cell systems (1.1) have a permutation symmetry $\sigma\left(x_{1}, x_{2}\right)=\left(x_{2}, x_{1}\right)$ inherited from the symmetry of the network. This symmetry causes synchrony and phase-locking to occur 'robustly', which means, roughly speaking, that these phenomena do not depend on the precise form of the function $g$ in (1.1).

In the case of synchrony, the reason for this independence of $g$ is very simple: the diagonal $\Delta=\left\{\left(x_{1}, x_{2}\right): x_{1}=x_{2}\right\}$ is a flow-invariant subspace for any choice of $g$. This is obvious, because the two equations in (1.1) reduce to the same equation on $\Delta$. It follows that if the initial conditions of a solution $x(t)$ satisfy $x_{1}(0)=x_{2}(0)$, then $x_{1}(t)=x_{2}(t)$ for all $t$. Two cells are synchronous if their time series are identical, so the invariance of $\Delta$ implies that synchrony should be expected in identical two-cell systems (1.1). Moreover, this synchrony is 'really' a consequence of symmetry. Indeed, suppose that $\gamma$ is a symmetry of (2.1). Then

$$
\operatorname{Fix}(\gamma)=\left\{x \in \mathbf{R}^{n}: x=\gamma x\right\}
$$


is a flow-invariant subspace. (Proof: $\gamma f(x)=f(\gamma x)=f(x)$ for each $x \in \operatorname{Fix}(\gamma)$. Hence $f: \operatorname{Fix}(\gamma) \rightarrow \operatorname{Fix}(\gamma)$.) The invariance of $\Delta$ can now be explained in symmetry terms, because $\Delta=\operatorname{Fix}(\sigma)$.

Phase-locking is also a natural consequence of symmetry, though it has a subtler cause, not directly related to a fixed-point space. To see how a phase-locked state can arise from symmetry, suppose that $x(t)$ is a $T$-periodic solution to (2.1) and that $\gamma$ is a symmetry. Then either $\gamma x(t)$ is a different periodic trajectory from $x(t)$ or it is the same trajectory. In the latter case, the only difference is a timetranslation. That is, $\gamma x(0)=x(\theta)$ for some $\theta$, and uniqueness of solutions implies that $\gamma x(t)=x(t+\theta)$ for all $t$. In the two-cell system, applying $\sigma$ twice implies that $2 \theta \equiv 0(\bmod T)$. Hence either $\theta=0$ or $\theta=\frac{T}{2}$. Since $\sigma$ is the permutation (1 2) it follows that $x_{2}(t)=x_{1}(t)$ when $\theta=0$ (synchrony) and $x_{2}(t)=x_{1}\left(t+\frac{T}{2}\right)$ when $\theta=\frac{T}{2}$ (phase-locking). Indeed, it is an important feature of identical two-cell models that these systems naturally produce in-phase (synchronous) periodic solutions and half-period out of phase (phase-locked) periodic solutions. The nephron model of Mosekilde et al. [60] can have periodic states that are a half-period out of phase. Biped gaits provide another excellent illustration: in-phase periodic states correspond to two-legged hopping (swapping left and right legs leaves the motion unchanged) and half-period out-of-phase periodic states correspond to walking. Animal gaits will be discussed in more detail in the next section.

These observations can be generalized substantially. The set of symmetries of an ODE is a group, so the natural context for symmetry is group theory. Most of the theory can be set up for a compact Lie group acting on a vector space or a manifold, and to some extent even for noncompact Lie groups, especially the Euclidean groups. However, the natural context for dynamics on finite networks, where we work with ODEs, is that of finite groups, and for simplicity we restrict the discussion to that case. For definiteness, we work with vector spaces as phase spaces. When the phase spaces are manifolds, new issues related to the global topology arise: see for example [31], which develops similar ideas for networks of phase oscillators.

Suppose, therefore, that the system (2.1) has a finite symmetry group $\Gamma$. First, suppose that $\Sigma \subset \Gamma$ is a subgroup. Then

$$
\operatorname{Fix}(\Sigma)=\left\{x \in \mathbf{R}^{n}: \sigma x=x \quad \forall \sigma \in \Sigma\right\}
$$

is a flow-invariant subspace. Second, suppose that $x(t)$ is a periodic solution. Define

$$
\begin{aligned}
& H=\{\gamma \in \Gamma: \gamma\{x(t)\}=\{x(t)\}\} \quad \text { spatiotemporal symmetries } \\
& K=\{\gamma \in \Gamma: \gamma x(t)=x(t) \quad \forall t\} \quad \text { spatial symmetries. }
\end{aligned}
$$

Then, as above, for each $h \in H$, there is a phase shift $\theta(h) \in \mathbf{S}^{1}$ such that $h x(t)=$ $x(t+\theta(h))$. Moreover, $\theta: H \rightarrow \mathbf{S}^{1}$ is a group homomorphism with kernel $K$. It follows that $H / K$ is isomorphic to a finite subgroup of $\mathbf{S}^{1}$ and hence is cyclic. Moreover, since fixed-point subspaces are flow-invariant, $K$ is an isotropy subgroup of the action of $\Gamma$ on $\mathbf{R}^{n}$.

Example 2.2. To illustrate the roles of $H$ and $K$, consider a ring of three identical cells with identical bidirectional coupling as in Figure 3. This network has a symmetry group $\mathbf{S}_{3}$ consisting of all permutations of the three cells (with corresponding permutations of the three arrows), and this group of network symmetries is reflected in the associated ODEs. Specifically, the systems of differential equations 
determined by this network have the form

$$
\begin{aligned}
& \dot{x}_{1}=g\left(x_{1}, \overline{x_{2}, x_{3}}\right) \\
& \dot{x}_{2}=g\left(x_{2}, \overline{x_{3}, x_{1}}\right) \\
& \dot{x}_{3}=g\left(x_{3}, \overline{x_{1}, x_{2}}\right)
\end{aligned}
$$

where $x_{1}, x_{2}, x_{3} \in \mathbf{R}^{k}$ and $g:\left(\mathbf{R}^{k}\right)^{3} \rightarrow \mathbf{R}^{k}$. The overline indicates that $g$ is invariant under permutation of the second and third coordinates, that is, $g(a, b, c) \equiv$ $g(a, c, b)$. This property reflects the fact that the two couplings affecting a single cell are identical.

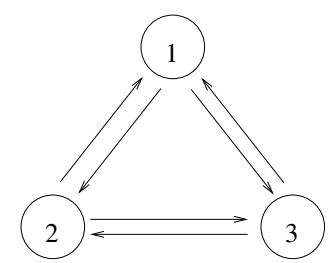

FiguRE 3. Schematic of a three-cell network with identical cells and identical coupling.

The symmetry group of this system is the permutation group $\Gamma=\mathbf{S}_{3}$ with generators $\sigma=\left(\begin{array}{lll}1 & 2\end{array}\right)$ and $\tau=\left(\begin{array}{lll}1 & 2 & 3\end{array}\right)$ in cycle notation (Biggs [8$)$. When $k \geq 2$, three different types of asynchronous periodic solutions can be found that have some spatiotemporal symmetry: rotating waves, two cells in phase, and two cells out of phase (by exactly half the period). These solution types are characterized by pairs $(H, K)$ equal to $\left(\mathbf{Z}_{3}(\tau), \mathbf{1}\right),\left(\mathbf{Z}_{2}(\sigma), \mathbf{Z}_{2}(\sigma)\right)$, and $\left(\mathbf{Z}_{2}(\sigma), \mathbf{1}\right)$, respectively. In rotating waves the time series of the three cells are identical up to a phase shift of one third or two thirds of a period. The out-of-phase periodic solution has a surprising feature. Suppose that $x(t)$ is a $T$-periodic solution satisfying $\sigma x(t)=x\left(t+\frac{T}{2}\right)$. Then $x_{2}(t)=x_{1}\left(t+\frac{T}{2}\right)$, as expected. However, in addition, $x_{3}(t)=x_{3}\left(t+\frac{T}{2}\right)$, so $x_{3}$ oscillates at twice the frequency of $x_{1}$ and $x_{2}$. Time series of rotating wave and out-of-phase periodic solutions are illustrated in Figure 4, which shows a numerical solution for a particular choice of $g$. Note the double-frequency small-amplitude time series in the right-hand figure.
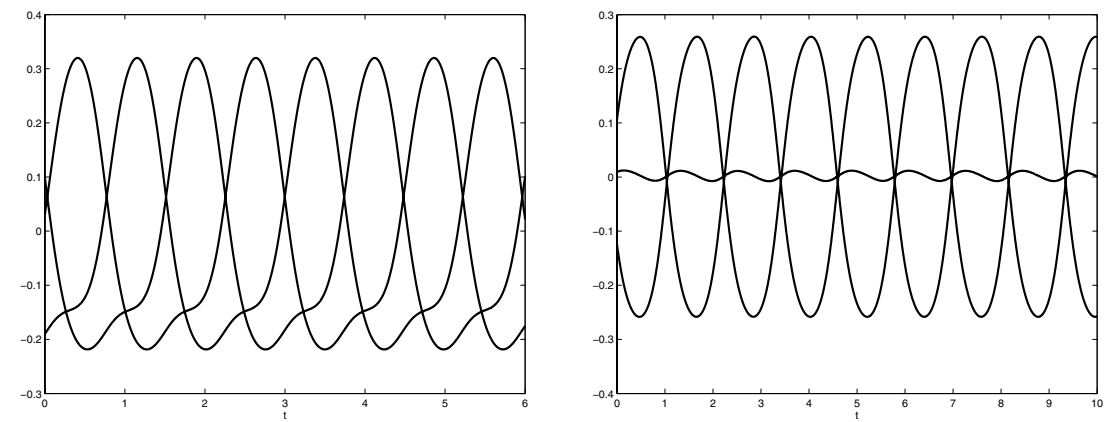

Figure 4. Periodic state of a three-cell network: (left) rotating wave, (right) out of phase. 
For a general coupled cell system, periodic states with spatiotemporal symmetries are classified by the following theorem.

Theorem 2.3 (H/K Theorem [13, 37]). Let $\Gamma$ be the symmetry group of a coupled cell network in which all cells are coupled and the internal dynamics of each cell is at least two-dimensional. Let $K \subset H \subset \Gamma$ be a pair of subgroups. Then there exist periodic solutions to some coupled cell system with spatiotemporal symmetries $H$ and spatial symmetries $K$ if and only if $H / K$ is cyclic and $K$ is an isotropy subgroup. Moreover, the system can be chosen so that the periodic solution is asymptotically stable.

Asymptotic stability implies hyperbolicity, which in turn implies that any small perturbation of the vector field leads to a unique perturbed periodic orbit that lies close to the original periodic orbit. It follows that both $H$ and $K$ are unchanged by small $\Gamma$-equivariant perturbations of the vector field. That is, the pattern of phase relations in the states whose existence is asserted by the $H / K$ theorem is 'rigid'unchanged by small perturbations. The same goes for the pattern of synchrony, since this corresponds to zero phase shifts. It is easy to construct examples of nonrigid phase relations, so this fact is nontrivial.

The implications of this theorem include some surprises. For example, consider the five-cell network in Figure 5. The symmetry group of this network is $\mathbf{Z}_{3} \times \mathbf{Z}_{2} \cong$ $\mathbf{Z}_{6}$. Periodic solutions with $(H, K)=\left(\mathbf{Z}_{6}, \mathbf{1}\right)$ can exist by the $H / K$ Theorem. Let

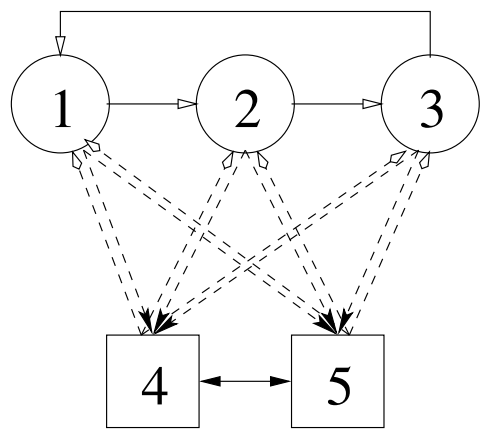

FiguRE 5. Five-cell system made from a ring of three cells and a ring of two cells.
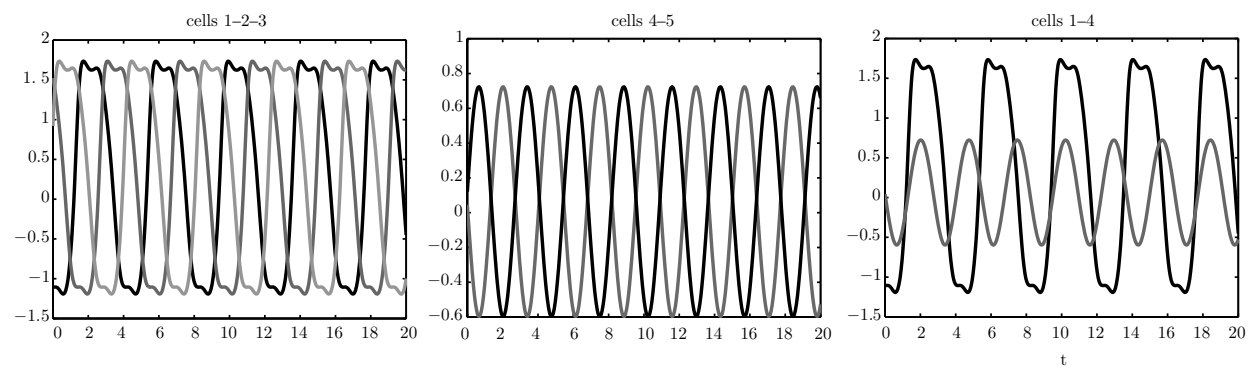

Figure 6. (L) Cells 1-2-3 one-third period out of phase; (C) cells 4-5 half period out of phase; $(\mathrm{R})$ shows that twice the frequency of cell 4 equals three times the frequency of cell 1 . 
$\sigma=(\rho, \tau)$ be the generator of $\mathbf{Z}_{3} \times \mathbf{Z}_{2}$. Observe that $\left(\sigma^{2}, 1 / 3\right)$ forces the 3-cell ring to exhibit a rotating wave and that $\left(\sigma^{3}, 1 / 2\right)$ forces the 2 -cell ring to generate out-of-phase signals. Finally, $(\sigma, 1 / 6)$ symmetry implies that three times the twocell frequency equals twice the three-cell frequency. Such a solution is shown in Figure 6. Such symmetry-induced 'resonances' are known as multirhythms.

\section{Animal Locomotion}

We apply the above ideas to a less trivial example, with some interest for applications, which has been suggested as a model for the patterns of synchrony and phase shifts that are observed in the movement of four-legged animals.

The general phenomenology of symmetric networks can be illustrated in the context of animal locomotion [13, 16, 17, 38, 39. It has long been recognized that legged locomotion involves a variety of standard spatio-temporal patterns in which the legs move periodically in a particular sequence and with particular phase relationships. The case of quadrupeds is especially familiar. For example, when a horse trots, diagonally opposed legs are synchronized, but the two diagonals are half a period out of phase. When the horse walks, the legs hit the ground in the sequence left rear, left front, right rear, right front (or its left/right mirror image) at intervals of one quarter period. When a camel or giraffe paces, its left legs are synchronous, its right legs are synchronous, but left and right are half a period out-of-phase. More complex gaits, such as the gallop, have phase shifts that are not such simple fractions of the period, leading to a distinction between primary gaits with very rigid, simple phase shifts, and secondary gaits with more arbitrary and more flexible phase shifts. (The symmetry formalism can be used to make this distinction precise, as we discuss shortly.) Figure 7 shows seven common quadruped gaits. Dogs tend to walk, trot, and transverse gallop; squirrels bound; camels tend to pace and rotary gallop; and deer often pronk (all legs moving in synchrony) when startled.

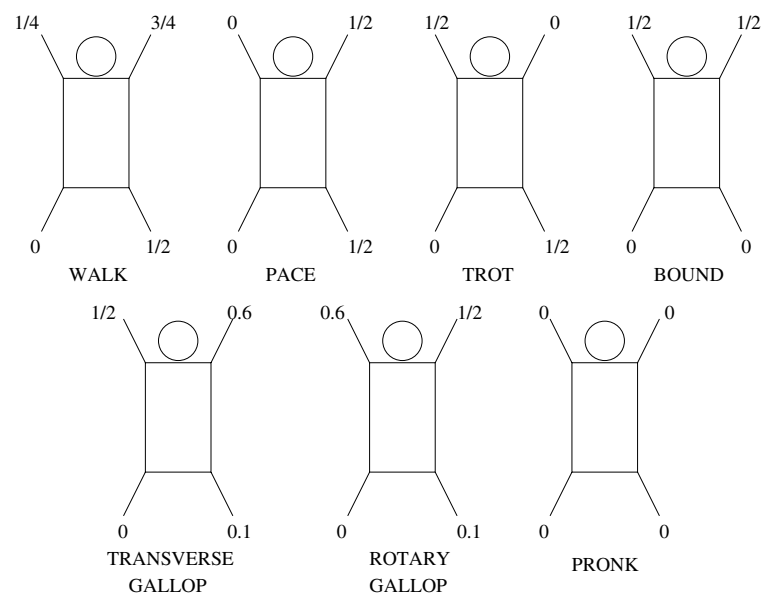

FiguRe 7. Seven quadrupedal gaits. Numbers indicate the percentage of the time through the gait when the associated leg first strikes the ground. Gaits begin when left hind leg strikes ground. 
The symmetry approach to gaits aims to provide a rationale for these patterns and to explain the distinction between primary and secondary gaits. It tackles these problems by seeking the schematic form of the animal's central pattern generator (CPG); see Kopell and Ermentrout [52. The CPG is a network of neurons that is widely believed to generate nerve signals with the corresponding spatio-temporal rhythms. Its existence is supported by much indirect evidence (see for example Grillner and Wallén 45), but significant information on the detailed structure of the CPG is known only for a few animals, notably the lamprey (see for example Grillner et al. [4]). For most animals even the existence of a CPG has not been confirmed directly, though it is well established that the basic rhythms of locomotion are generated somewhere in the spinal cord, not in the brain. It therefore makes sense to try to infer qualitative information about the CPG from the gaits themselves.

Such inferences must start by making some assumptions about the nature of the CPG and how it relates to the gaits, and the consequent deductions are only as good as those assumptions. So what should emerge is either a valid CPG architecture or the falsification of at least one of the assumptions. In the current state of knowledge, either of these is possible, but what concerns us here is the use of symmetry arguments to infer features of network topology, and conversely.

An important but sometimes neglected aspect of gaits is the phase shifts that do not occur, as well as the ones that do. This rules out otherwise effective 'engineering' designs of CPG that can produce any desired collection of phase shifts.

Four Cells Do Not Suffice. The simplest model of a quadruped locomotor CPG has four identical cells, where it is presumed that the output signal from each cell is sent to one leg. See Figure 8, We ask whether it is possible to couple these four cells in such a way that network systems can naturally produce rhythms associated with the three gaits - walk, trot, and pace - and show that it is not [13.

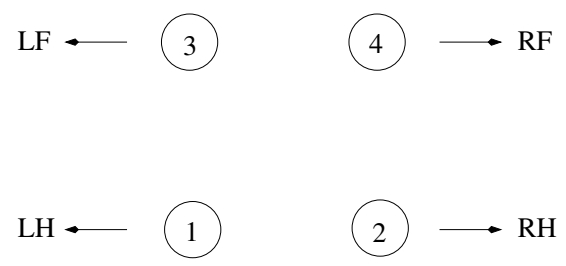

Figure 8. Signal from cell 1 is sent to left hind (LH) leg, etc.

To justify this negative statement we discuss three points:

(a) Gait rhythms are described by spatiotemporal symmetries.

(b) The symmetry groups of trot and pace cannot be conjugate.

(c) The symmetry group of trot and pace are always conjugate in any four-cell network that also produces a walk.

(a) Collins and Stewart [17 observed that standard quadruped gaits are distinguished by spatiotemporal symmetries, where the space symmetries are leg permutations. The generators for the symmetry groups of trot, pace, and walk are listed in Table 1. In our models we assume that gait rhythms are exact and robust. We also assume that the only robust phase shifts of periodic solutions that are given in these models are those that are described by symmetry. This point will be discussed further in Section 10 , 


\begin{tabular}{|c|c|c|}
\hline Gait & Generators of spatio-temporal symmetries & Solution form \\
\hline Trot & 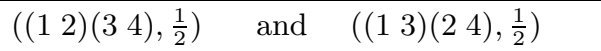 & $\left(x(t), x\left(t+\frac{1}{2}\right), x\left(t+\frac{1}{2}\right), x(t)\right)$ \\
\hline Pace & $\left((12)(34), \frac{1}{2}\right) \quad$ and $\quad\left(\left(\begin{array}{l}1 \\
1\end{array}\right)(24), 0\right)$ & $\left(x(t), x\left(t+\frac{1}{2}\right), x(t), x\left(t+\frac{1}{2}\right)\right)$ \\
\hline Walk & $\left(\left(\begin{array}{llll}1 & 3 & 2 & 4\end{array}\right), \frac{1}{4}\right)$ & $\left(x(t), x\left(t+\frac{1}{2}\right), x\left(t+\frac{1}{4}\right), x\left(t+\frac{3}{4}\right)\right)$ \\
\hline
\end{tabular}

TABLE 1. Legs are numbered by the associated cells in Figure 8 , The permutation (1 2)(3 4) swaps left and right legs; the permutation $\left(\begin{array}{lll}1 & 3\end{array}\right)(24)$ swaps front and back legs; fractions indicate phase shift as a fraction of a gait period.

(b) Experiments on dogs imply that trot and pace are not gaits that can be modeled by conjugate solutions. Note that in a system of differential equations conjugate solutions differ only by initial conditions and have the same stability. Blaszczyk and Dobrzecka 9 indicate that the stability of pace and trot are not the same. In their experiment, a dog's legs are restrained so that they can use a pace at intermediate speeds, but not a trot, which is the dog's preferred gait. Different dogs are placed in this device for two to six months. In post-restraint trials dogs that were in the shorter restraint period switched back to a trot quickly with only occasional use of a pace. Occurrence of the pace was more frequent in the animals that were restrained for a longer period, but the use of pace decreased with every post-restraint experimental trial.

(ㄷ) It follows from (a) that if a four-cell network is coupled so that periodic solutions with the rhythm of a walk occur naturally, then the permutation (1 $\left.\begin{array}{llll}1 & 3 & 2 & 4\end{array}\right)$ must be a network symmetry. Suppose that the system also produces a pace solution. As indicated in Figure 9, cells 1 and 3 and cells 2 and 4 must be synchronous. As illustrated in the figure, applying the walk symmetry to that solution produces a solution in which cells 1 and 4 and cells 2 and 3 are synchronous - a pace. It follows that trot and pace solutions are conjugate in any four-cell network that can produce a robust walk.
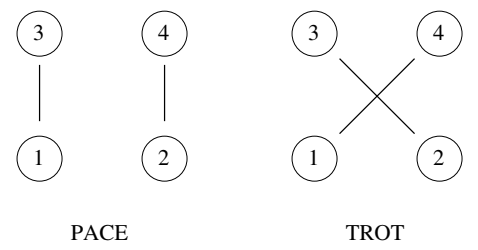

Figure 9. Lines between cells indicate synchrony; no lines indicate half-period phase shifts.

The Eight-Cell Network. Golubitsky et al. [13, 39] make six assumptions and deduce that for quadrupeds the only possible symmetry class of CPG networks is the 8-cell network shown in Figure10. The details of the deduction are unimportant here, but they are explicit in the original paper. In this figure, unlike all the others in this paper, the edges of the graph do not represent specific connections but compose the simplest set of edges that determines the network's symmetry group. If any additional edge is added, all symmetrically related edges must be added as 


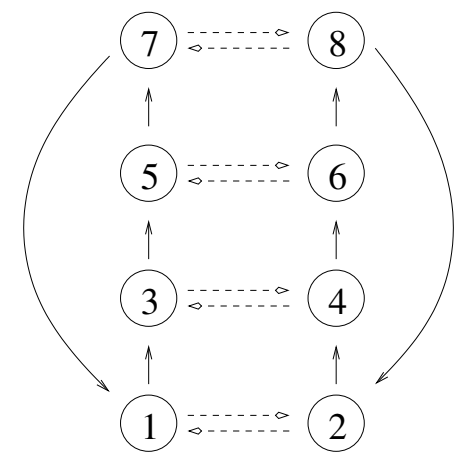

Figure 10. Eight-cell network for quadrupeds. Dashed lines indicate contralateral coupling; single lines indicate ipsilateral coupling.

well. For example, if we add an edge from cell 1 to cell 4 , then we must also add identical edges from cells 3 to 6,5 to 1,7 to 2,2 to 3,4 to 5,6 to 7 , and 8 to 1 . We adopt this convention for this example only, because otherwise the picture would become too complicated to follow.

It is worth remarking that the context assumed in the analysis was that of group-equivariant dynamical systems, leaving open the possibility that groupoid symmetries might lead to an alternative, smaller, network with similar properties. There are good reasons to believe that this is not the case, but we postpone this issue to Section 10, by which point the necessary ideas will be available.

Perhaps the most surprising feature here is that a 4-cell network, with one cell per leg, is ruled out. The reason is related to the walk, trot, and pace gaits and how they coexist.

This network has eight symmetries: permutations of the legs (more precisely, the leg labels) that preserve the edges. There are two types of symmetry: contralateral symmetry $\kappa$, which interchanges cells on the left with cells on the right; and ipsilateral symmetry $\omega$, which cyclically and simultaneously permutes cells on both left and right. Thus the symmetry group of the eight-cell quadruped CPG is $\Gamma=\mathbf{Z}_{2}\langle\kappa\rangle \times \mathbf{Z}_{4}\langle\omega\rangle$.

The $H / K$ Theorem provides a classification of the possible spatio-temporal symmetries. Primary states are characterized by all eight cells having the same waveform modulo phase shift (that is, $H=\Gamma$ ), whereas secondary gaits involve more

\begin{tabular}{|c|c|c|c|c|c|c|c|c|c|}
\hline & & & $\mathrm{lk}$ & jump & $\operatorname{tr}$ & ot & pace & bound & pronk \\
\hline $\mathrm{LF}$ & $\mathrm{RF}$ & & $\frac{1}{4}$ & $\frac{1}{2} \quad \frac{1}{2}$ & & & $0 \quad \frac{1}{2}$ & $\frac{1}{2}$ & $\begin{array}{ll}0 & 0\end{array}$ \\
\hline LH & $\mathrm{RH}$ & & 0 & $\begin{array}{ll}\frac{3}{4} & \frac{3}{4}\end{array}$ & 0 & $\frac{1}{2}$ & 0 & 0 & $\begin{array}{ll}0 & 0\end{array}$ \\
\hline LF & $\mathrm{RF}$ & $\frac{1}{4}$ & $\frac{3}{4}$ & $\begin{array}{ll}0 & 0\end{array}$ & & 0 & 0 & $\frac{1}{2}$ & $\begin{array}{ll}0 & 0\end{array}$ \\
\hline LH & $\mathrm{RH}$ & 0 & $\frac{1}{2}$ & $\frac{1}{4}$ & 0 & $\frac{1}{2}$ & 0 & 0 & $\begin{array}{ll}0 & 0\end{array}$ \\
\hline $\mathrm{Sul}$ & ip $K$ & $\mathbf{Z}_{2}$ & $\left.i \omega^{2}\right)$ & $\overline{\overline{\mathbf{Z}_{2}(\kappa)}}$ & $\mathbf{Z}_{4}$ & $\overline{\kappa \omega)}$ & $\mathbf{Z}_{4}(\omega)$ & $\overline{\mathbf{D}_{2}\left(\kappa, \omega^{2}\right)}$ & $\mathbf{Z}_{2} \times \mathbf{Z}$ \\
\hline
\end{tabular}

TABLE 2. Phase shifts for primary gaits in the eight-cell network. 
than one waveform (that is, $H \subsetneq \Gamma$ ). It is straightforward to calculate the six subgroups $K \subset H$ for which $H / K$ is cyclic and to determine the primary patterns for the 8-cell network; see Table 2 There is an analogous (but more complicated) classification of secondary gaits.

The patterns listed in the table correspond to standard primary quadruped gaits, with one exception: the gait we have labelled 'jump'. After performing the above analysis, the jump gait was observed at the Houston Livestock Show and Rodeo. Figure 11 shows four video frames of a bucking bronco, taken at equal intervals of time. The interval between the footfalls is very close to $1 / 4$ of the period of this rhythmic motion.
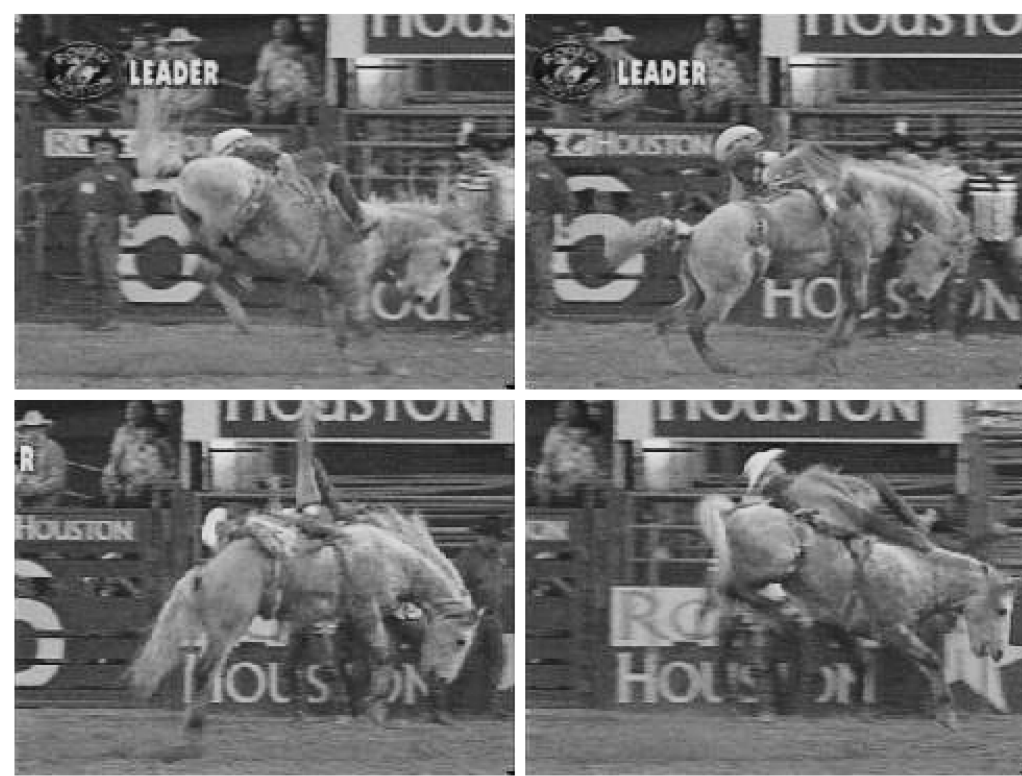

FiguRE 11. Quarter cycles of bareback bronc jump at Houston Livestock Show and Rodeo. (UL) fore legs hit ground, (UR) hind legs hit ground, (LL) and (LR) all legs in air.

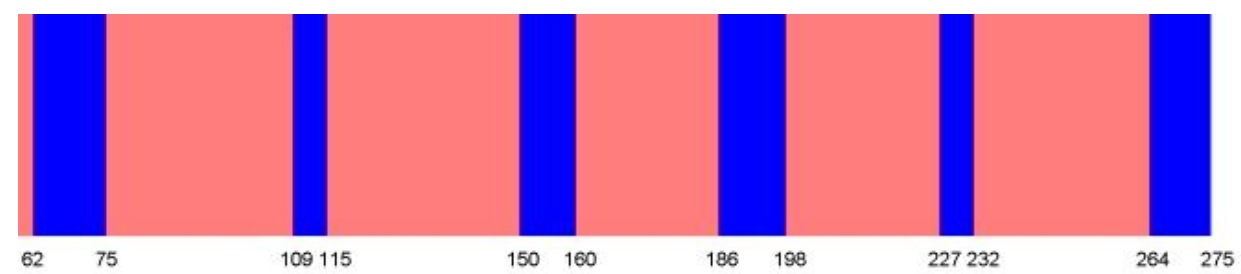

FiguRE 12. Average right hind to right fore $=31.2$ frames (light region); average right fore to right hind $=11.4$ frames (dark region); $\frac{31.2}{11.4}=2.74$.

Indeed, approximately 200 frames of the rodeo video are coded in Figure 12 , Dark regions begin when the right hind leg is firmly on the ground and light regions 
begin when the right fore leg is firmly planted on the ground. This figure indicates that the average time elapsed from right hind to right fore leg ground strikes is approximately three times the average time elapsed from right fore to right hind leg ground strikes. The primitive ricocheting jump of a Norway rat and an Asia Minor gerbil also has the same pattern of phases as the jump gait (Gambaryan [29]).

\section{IS SYMMETRY NECESSARY FOR SYNCHRONY?}

The theory of symmetric dynamical systems shows that striking patterns, such as synchrony and phase-relations, are to be expected in systems with a global group of symmetries. It also provides general theorems and techniques for deriving the appropriate patterns for any given symmetry group. However, symmetry in the group-theoretic sense is a rather strong modeling assumption.

The question here is not so much whether symmetry is 'realistic', but what kind of symmetry is appropriate. If a realistic model is 'close to' an idealized symmetric one, then (invoking normal hyperbolicity) we can anticipate that in many respects the realistic model's behavior will resemble that of the symmetric ideal. Some delicate features will not survive the slight loss of symmetry, but the more robust features will. For example, if the idealized model predicts that two variables will be exactly synchronous, then the corresponding variables of a more realistic model will track each other fairly closely, even though their values may differ slightly.

Many models, in particular networks, do not possess global group-theoretic symmetries, even in this approximate sense. So it is reasonable to ask whether global group-theoretic symmetry is necessary for rigid synchrony and phase-locking, as well as being sufficient and natural.

The perhaps surprising answer is 'no', and this fact forms the basis of the theory to be presented in this article.

The observation that rigid synchrony and phase relations can occur in a more general class of networks leads us to relax the constraint that the network should have group-theoretic symmetry and to begin compiling evidence that a groupoidtheoretic generalization is also natural, as well as being mathematically interesting and potentially useful in applications. (We emphasize the word 'potentially'.)

In the remainder of this section we justify the claim that rigid synchrony and phase relations can occur in networks whose symmetry group is trivial by presenting some simple examples where such features arise. We also explain why these features are present. These explanations motivate the formal theory developed in Sections 5 7.

We begin by showing that network symmetry is not needed for synchrony and phase-locking and derive the idea of a 'quotient network'. We set the scene by discussing four examples. These examples have been chosen to illustrate mathematical points and have no particular significance for applications.

Example 4.1. Figure 13 shows a directed ring of 3 identical cells with identical couplings. The associated ODEs are $\mathbf{Z}_{3}$-equivariant and take the form

$$
\begin{aligned}
& \dot{x_{1}}=g\left(x_{1}, x_{3}\right) \\
& \dot{x_{2}}=g\left(x_{2}, x_{1}\right) \\
& \dot{x_{3}}=g\left(x_{3}, x_{2}\right)
\end{aligned}
$$




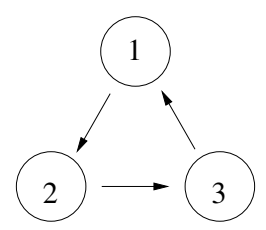

Figure 13. A 3-cell unidirectional ring.

As for the bidirectional ring (Figure 3), $H / K$ theory suggests that a unidirectional ring can support periodic states $x(t)$ in which

$$
\begin{aligned}
& x_{2}(t)=x_{1}(t+T / 3) \\
& x_{3}(t)=x_{1}(t+2 T / 3)
\end{aligned}
$$

where $T$ is the period. Such a state is called a (discrete) rotating wave. Fully synchronous states can also occur. However, the unidirectional ring does not support the double frequency periodic state (see Figure 4 (right)) found in the bidirectional ring. Indeed, there is no pair of subgroups $H, K$ in $\mathbf{Z}_{3}$ that can correspond to such a state.

Example 4.2. Next, consider a linear chain of (seven) identical cells; see Figure14, This network has trivial symmetry. The associated ODEs have the form

$$
\begin{aligned}
& \dot{x_{1}}=h\left(x_{1}\right) \\
& \dot{x_{j}}=g\left(x_{j}, x_{j-1}\right) \quad j=2, \ldots, 7
\end{aligned}
$$

and thus involve two distinct functions, $g$ and $h$. Despite the regular form of the network, traveling waves solutions are not expected (and in fact cannot occur if the cell phase spaces are all 1-dimensional).

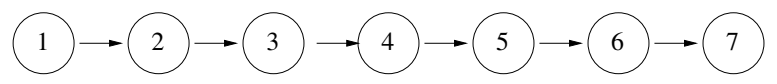

FiguRE 14. Seven-cell linear network.

Example 4.3. Modify the previous example by inserting a feedback loop; see Figure 15. The symmetry group remains trivial, but the associated ODEs become

$$
\begin{aligned}
\dot{x_{1}} & =g\left(x_{1}, x_{3}\right) \\
\dot{x_{j}} & =g\left(x_{j}, x_{j-1}\right) \quad j=2, \ldots, 7
\end{aligned}
$$

so that the same function $g$ occurs in every equation.

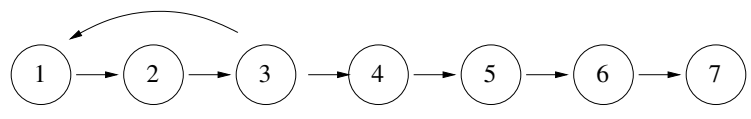

Figure 15. A seven-cell chain.

This chain network is closely related to the unidirectional ring network of Figure 13. In fact, if we restrict attention to states in which

$$
\begin{aligned}
& x_{1}=x_{4}=x_{7} \\
& x_{2}=x_{5} \\
& x_{3}=x_{6}
\end{aligned}
$$


then equations (4.3) become exactly the same as (4.1). It follows that the "polydiagonal' $\Delta=\left\{\left(x_{1}, x_{2}, x_{3}, x_{1}, x_{2}, x_{3}, x_{1}\right): x_{1}, x_{2}, x_{3} \in \mathbf{R}\right\}$ is flow-invariant for the chain network in Figure 15. for any choice of $g$. Hence synchrony is possible even though no network symmetry is present. It also follows that we can 'lift' any rotating wave state of the 3-cell unidirectional ring to the 7-cell chain, obtaining a state in which

$$
\begin{aligned}
& x_{7}(t)=x_{4}(t)=x_{1}(t) \\
& x_{6}(t)=x_{3}(t)=x_{1}(t+2 T / 3) \\
& x_{5}(t)=x_{2}(t)=x_{1}(t+T / 3) .
\end{aligned}
$$

This is a traveling wave, consisting of three synchronous sets of cells with phaseshifts $0, T / 3,2 T / 3$. Thus nontrivial phase-locking is present even though no network symmetry exists.

We say that the unidirectional ring network in Figure 13 is a quotient network of the chain network in Figure 15. Section 9 develops this useful notion.

Example 4.4. The fourth example, Figure 16, is a 5-cell network. This network consists of a directed ring of four cells, forced symmetrically by a fifth cell (and would therefore have $\mathbf{Z}_{4}$ symmetry if there were no other connections). However, there is also feedback from two cells in the ring to the forcing cell, which breaks the $\mathbf{Z}_{4}$ symmetry. The equations that correspond to the network take the form

$$
\begin{aligned}
& \dot{x}_{1}=g\left(x_{1}, \overline{x_{4}, x_{5}}\right) \\
& \dot{x}_{2}=g\left(x_{2}, \overline{x_{1}, x_{5}}\right) \\
& \dot{x}_{3}=g\left(x_{3}, \overline{x_{2}, x_{5}}\right) \\
& \dot{x}_{4}=g\left(x_{4}, \overline{x_{3}, x_{5}}\right) \\
& \dot{x}_{5}=g\left(x_{5}, \overline{x_{2}, x_{3}}\right)
\end{aligned}
$$

where the 'overline' indicates that the couplings are interchangeable, that is, $g(a, b, c)=g(a, c, b)$. We show that this network has a symmetric quotient network and a quotient network with self-coupling and multiple arrows.

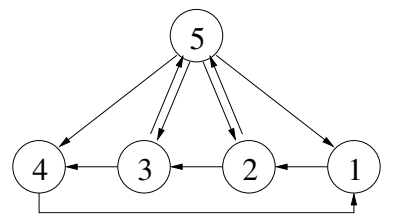

FiguRE 16. Five-cell network.

We define a polysynchronous subspace of a coupled cell network to be a flowinvariant polydiagonal. Perhaps surprisingly, this network has five polysynchronous subspaces. Each of them corresponds to a partition of the set of cells - a decomposition into disjoint subsets - such that the components of the state vector are identical (that is, the cells are synchronous) on each subset in the partition. See Table 3. We call such a partition a pattern of synchrony of the cells.

Each of these five patterns of synchrony leads to a system of restricted ODEs. For example, if we take the partition $\{1,3\}\{2,4\},\{5\}$ and the corresponding subspace $\{x, y, x, y, z\}$, then the restricted equations take the form:

$$
\begin{aligned}
& \dot{x}=g(x, \overline{y, z}) \\
& \dot{y}=g(y, \overline{x, z}) \\
& \dot{z}=g(z, \overline{y, x}) .
\end{aligned}
$$




\begin{tabular}{|c|c|}
\hline partition & polysynchronous subspace \\
\hline \hline$\{1,2,3,4,5\}$ & $\{x, x, x, x, x\}$ \\
$\{1,2,3,4\},\{5\}$ & $\{x, x, x, x, y\}$ \\
$\{1,3,5\},\{2,4\}$ & $\{x, y, x, y, x\}$ \\
$\{1,3\},\{2,4,5\}$ & $\{x, y, x, y, y\}$ \\
$\{1,3\},\{2,4\},\{5\}$ & $\{x, y, x, y, z\}$ \\
\hline
\end{tabular}

TABLE 3. Synchrony patterns for Figure 16

These correspond precisely to a 3-cell bidirectional ring network with symmetry group $\mathbf{S}_{3}$, shown in Figure 3. So this asymmetric 5-cell network has a symmetric quotient network. This 'concealed' symmetry has dynamical consequences for the five-cell network. We know that for suitable choices of the ODE, the 3-cell ring can have periodic states where one cell oscillates with twice the frequency of the other two (recall Figure4(right)). We can lift this state from the three-cell quotient network to the original five-cell network, leading to a state shown in Figure17. Note that the left image has cell 5 oscillating at double frequency and the right image has cells 2 and 4 oscillating synchronously and at double frequency. Remarkably, because of the $\mathbf{S}_{3}$ symmetry in (4.5), these solutions, which look so different in cell coordinates, are obtained merely by choosing different initial conditions in the appropriate synchrony subspace.
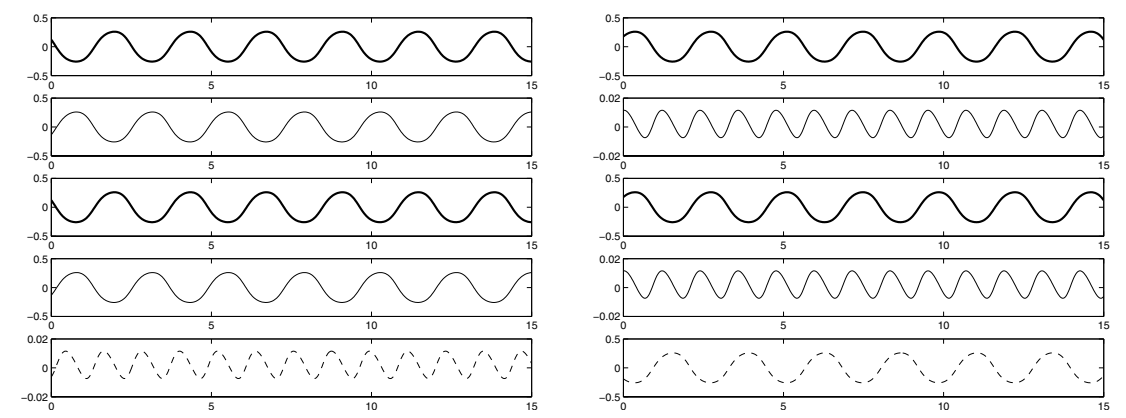

Figure 17. Double frequency solutions in five-cell network.

Moreover, the stabilities of these two solutions, inside the synchrony subspace, are the same. However, the stabilities transverse to the synchrony subspace are in general different (though both solutions happen to be stable for this particular choice of parameters). To analyse the transverse stability, consider (4.4). Use the notation $g(i j k)=g\left(x_{1}, x_{j}, x_{k}\right)$ and recall that $g$ is symmetric in the second and third arguments. So

$$
g_{v}(i j k)=g_{w}(i k j)
$$

Let $a=\left(x_{1}, x_{2}, x_{1}, x_{2}, x_{5}\right)$ and $b=\left(x_{2}, x_{5}, x_{2}, x_{5}, x_{1}\right)$ be $\mathbf{S}_{3}$ related equilibria in the synchrony subspace. We compute the transverse eigenvalues of the Jacobians $J_{a}$ 
and $J_{b}$ using the basis

$$
v_{1}=\left[\begin{array}{l}
1 \\
0 \\
1 \\
0 \\
0
\end{array}\right] \quad v_{2}=\left[\begin{array}{l}
0 \\
1 \\
0 \\
1 \\
0
\end{array}\right] \quad v_{3}=\left[\begin{array}{l}
0 \\
0 \\
0 \\
0 \\
1
\end{array}\right] \quad v_{4}=\left[\begin{array}{c}
1 \\
0 \\
-1 \\
0 \\
0
\end{array}\right] \quad v_{5}=\left[\begin{array}{c}
0 \\
1 \\
0 \\
-1 \\
0
\end{array}\right]
$$

since $v_{1}, v_{2}, v_{3}$ is a basis for the synchrony subspace. The eigenvalues in the transverse directions are found by computing $J_{a}\left(v_{4}\right), J_{a}\left(v_{5}\right), J_{b}\left(v_{4}\right)$, and $J_{b}\left(v_{5}\right)$. A calculation shows that the transverse eigenvalues are the eigenvalues of the matrices

$$
M_{a}=\left[\begin{array}{cc}
g_{u}(125) & -g_{v}(125) \\
g_{v}(215) & g_{u}(215)
\end{array}\right] \quad \text { and } \quad M_{b}=\left[\begin{array}{cc}
g_{u}(251) & -g_{v}(251) \\
g_{v}(521) & g_{u}(521)
\end{array}\right] .
$$

There is no reason for these matrices to be related, and the eigenvalues and stabilities can certainly be different.

The system (4.4) also illustrates another phenomenon, which turns out to be important in setting up a suitable formal theory. Consider the polysynchronous subspace $\{x, y, x, y, y\}$. On this subspace system (4.4) reduces to the system

$$
\begin{aligned}
& \dot{x}=g(x, \overline{y, y}) \\
& \dot{y}=g(y, \overline{x, y})
\end{aligned} .
$$

In a natural way, to be explained more formally in later sections, this restricted system corresponds to the network in Figure 18 with self-coupling and multiple arrows. This hints that our concept of 'network' should permit these two features. $\diamond$

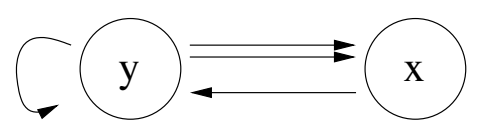

FiguRE 18. Two-cell quotient network with self-coupling and multiple arrows.

Not every identification of cells leads to a consistent set of equations, hence a possible pattern of synchrony. For example, if we identify cells according to the partition $\{1,2,3\},\{4,5\}$ with associated variables $y, z$ and polydiagonal $\{y, y, y, z, z\}$, then the reduced equations become

$$
\begin{aligned}
& \dot{y}=g(y, \overline{z, z}) \\
& \dot{y}=g(y, \overline{y, z}) \\
& \dot{y}=g(y, \overline{y, z}) \\
& \dot{z}=g(z, \overline{y, z}) \\
& \dot{z}=g(z, \overline{y, y}) .
\end{aligned}
$$

The first equation does not match the second and third, and the fourth and fifth do not match either. In other words, the space $Y=\{(y, y, y, z, z)\}$ is not flowinvariant for all ODEs of the form (4.4). In particular, we do not expect to see a robust pattern of synchrony in which cells $1,2,3$ are synchronous and cells 4,5 are synchronous. 
What property of the network characterizes the polysynchronous subspaces? To answer this basic question we must first formalize the idea of a network and the associated class of ODEs. We devote the next two sections to this task.

\section{Coupled Cell Networks}

In order to analyze how the dynamics of networks relates to the network architecture, we must first specify what we mean by a network and what dynamics we consider to be consistent with its architecture. We will then be in a position to prove rigorous theorems and ask precise questions about that relationship.

In this section we define a coupled cell network and focus attention on the 'input sets' of the cells - namely, those cells from which they receive coupling and whose associated variables appear in the component of the ODE that governs the dynamic of the chosen cell. Cells whose input sets consist of similar types of cells, with similar couplings, should obey similar equations. More precisely, if some permutation of the cells and couplings preserves the architecture of the input sets, then the corresponding components of the ODE should involve the same function, but with suitably permuted variables. This simple and natural observation leads, inexorably, to the conclusion that the structure of the ODE is determined by the 'symmetry groupoid' of the network.

We hasten to add that we do not require the abstract theory of groupoids, as described in, say, Higgins [47. Our groupoids will be very concrete: sets of permutations, acting on certain subsets of cells. However, the groupoid framework does lead to conditions on the ODE that naturally generalize group-equivariance, making it convenient to use groupoid terminology.

There are several ways to formalize the structure of a coupled cell network and the associated ODEs. Our philosophy when setting up the formal theory is to avoid artificial restrictions such as linearity, weak coupling, and additive coupling. One reason is that these assumptions seldom survive an appropriate change of coordinates. However, they are often useful to motivate the formal theory and can be entirely appropriate in specific models. Because we wish to understand the influence of network architecture on dynamics, we prefer not to make strong assumptions about the type of dynamics that may occur. We therefore seek the most general class of ODEs that conforms to the network architecture. (Because a major focus is local bifurcation, we find it convenient to assume that the phase space is a vector space, but this assumption is not essential; see for example Section [13.)

As a matter of recent history, the original formulation in 72 was quickly superseded by a slightly more complicated version, introduced in [42, which is nonetheless preferable, because it has useful technical advantages. We motivate the definition, state it, and then explain the advantages. The associated ODEs are treated in the next section.

The state of cell $c$ at time $t$ is determined by a list of state variables for that cell. (For ODE models the list is finite.) Each cell $c$ must be assigned a cell phase space (or cell state space) $P_{c}$, and its state $x_{c}(t)$ at time $t$ is an element of $P_{c}$. In general $P_{c}$ should be a manifold, and for simplicity we take it to be a finite-dimensional real vector space.

Each cell typically has an internal dynamic, an ODE that determines its behavior in isolation. In a network, the internal dynamic of a given cell is modified by coupling effects from other cells. The direction of coupling is important since it 
determines which cell is affected by which. It is therefore natural, and common, to represent the cells as the nodes of a directed graph (or digraph, Tutte [76], Wilson [84]) and the couplings as directed edges, drawn as arrows.

In many applications the cells occur in a variety of different types, and cells of the same type have the same internal dynamic (expressed in suitable coordinates). There are two ways to formalize this requirement. One is to attach labels to cells, symbols drawn from some finite set. Cells with identical labels are required to have the same type. Alternatively, we can introduce an equivalence relation 'same type' on cells. Logically, both approaches are equivalent, but psychologically each has advantages in some contexts. For example, it is visually useful to label the cells in the network diagram by the shape of the symbol used for a given node (circle, square, hexagon, and so on), but for formal purposes it is more convenient to introduce a relation of 'cell-equivalence', which does the same job.

Similarly, coupling among cells also occurs in various distinguishable types. We can 'label' arrows graphically by using different kinds of lines (solid, dashed, dotted) and/or different arrowheads (black, white), but for formal purposes we introduce a relation of 'edge-equivalence'.

Figure 19 presents a 3-cell example with two cell types (cells 1 and 2 have the same type, but cell 3 is different) and three edge types (the couplings from cells 2 to 1 and 1 to 2 are the same, and the couplings from cell 3 to cells 1 and 2 are the same). Systems of ODEs associated with this network have the form:

$$
\begin{aligned}
& \dot{x}_{1}=g\left(x_{1}, x_{2}, x_{3}\right) \\
& \dot{x}_{2}=g\left(x_{2}, x_{1}, x_{3}\right) \\
& \dot{x}_{3}=h\left(x_{3}, x_{1}\right) .
\end{aligned}
$$

Note that the same function $g$ appears in cells 1 and 2. This $g$ has no special symmetry properties.

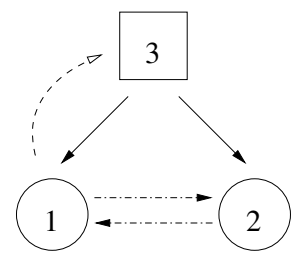

Figure 19. A 3-cell example with two cell types and three edge types.

Finally, we follow the normal conventions of graph theory and represent the topology of the network by two 'incidence relations', $\mathcal{H}$ and $\mathcal{T}$, which determine the cells that lie at the head and tail of a given arrow (Tutte [76], Wilson [84]).

Thus motivated, we can now state:

Definition 5.1. A coupled cell network $G$ comprises:

(a) A finite set $\mathcal{C}=\{1, \ldots, N\}$ of nodes or cells.

(b) An equivalence relation $\sim_{C}$ on cells in $\mathcal{C}$.

The type or cell label of cell $c$ is the $\sim_{C}$-equivalence class $[c]_{C}$ of $c$.

(c) A finite set $\mathcal{E}$ of edges or arrows.

(d) An equivalence relation $\sim_{E}$ on edges in $\mathcal{E}$.

The type or coupling label of edge $e$ is the $\sim_{E}$-equivalence class $[e]_{E}$ of $e$. 
(e) Two maps $\mathcal{H}: \mathcal{E} \rightarrow \mathcal{C}$ and $\mathcal{T}: \mathcal{E} \rightarrow \mathcal{C}$.

For $e \in \mathcal{E}$ we call $\mathcal{H}(e)$ the head of $e$ and $\mathcal{T}(e)$ the tail of $e$.

We also require a consistency condition:

(f) Equivalent arrows have equivalent tails and heads. That is, if $e_{1}, e_{2} \in \mathcal{E}$ and $e_{1} \sim_{E} e_{2}$, then

$$
\mathcal{H}\left(e_{1}\right) \sim_{C} \mathcal{H}\left(e_{2}\right) \quad \mathcal{T}\left(e_{1}\right) \sim_{C} \mathcal{T}\left(e_{2}\right) .
$$

Two noteworthy features of this definition are:

- Self-coupling is permitted: that is, we allow $\mathcal{H}(e)=\mathcal{T}(e)$ for an edge $e$.

- Multiple arrows are permitted: we allow $\mathcal{H}\left(e_{1}\right)=\mathcal{H}\left(e_{2}\right)$ and $\mathcal{T}\left(e_{1}\right)=\mathcal{T}\left(e_{2}\right)$ for $e_{1} \neq e_{2}$. (We even permit $e_{1}, e_{2}$ to have different edge-types.)

Example 4.4 shows why these features are useful, and other examples reinforce that view. See 42 for details.

Associated with each cell $c \in \mathcal{C}$ is a canonical set of edges, namely, those that represent couplings into cell $c$ :

Definition 5.2. If $c \in \mathcal{C}$, then the input set of $c$ is

$$
I(c)=\{e \in \mathcal{E}: \mathcal{H}(e)=c\} .
$$

An element of $I(c)$ is called an input edge or input arrow of $c$.

Input edges determine the form of the ODEs associated with $G$. For a given cell $c \in \mathcal{C}$, the form of $\dot{x}_{c}$ should depend only on the cells coupled to cell $c$, that is, on $x_{c}$ and on those $x_{i}$ for which there exists an arrow with head $c$ and tail $i$. This point will be discussed in more detail in Section 6. Figure 20 illustrates the input sets of the 3-cell network in Figure 19. Observe that after relabeling, the input sets of cells 1 and 2 are identical. Their common input structure reflects the occurrence of the same function $g$ in the ODEs for cells 1 and 2 .

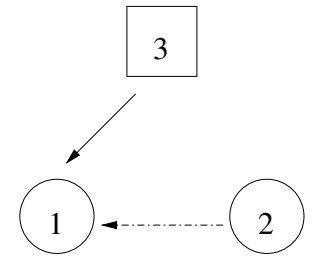

Cell 1

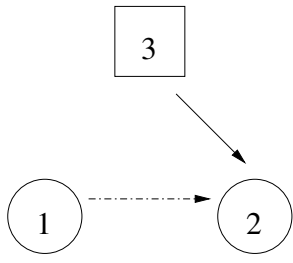

Cell 2

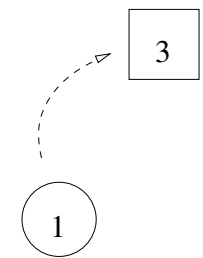

Cell 3

Figure 20. Input sets for 3-cell example in Figure 19 .

Definition 5.3. The relation $\sim_{I}$ of input equivalence on $\mathcal{C}$ is defined by $c \sim_{I} d$ if and only if there exists an arrow type preserving bijection

$$
\beta: I(c) \rightarrow I(d) \text {. }
$$

That is, for every input arrow $i \in I(c)$

$$
i \sim_{E} \beta(i) .
$$


Any such bijection $\beta$ is called an input isomorphism from cell $c$ to cell $d$. The set $B(c, d)$ denotes the collection of all input isomorphisms from cell $c$ to cell $d$. The set

$$
\mathcal{B}_{G}=\bigcup_{c, d \in \mathcal{C}} B(c, d)
$$

is the (symmetry) groupoid of the network. The set $B(c, c)$ is a permutation group acting on the input set $I(c)$, which we call the vertex group of cell $c$.

A groupoid (Brandt 11, Brown [12, Higgins 47]) is an algebraic structure rather like a group, except that the product of two elements is not always defined. Note that the union in (5.5) is disjoint. By the consistency condition (f) of Definition 5.1, $c \sim_{I} d$ implies $c \sim_{C} d$, but the converse fails in general.

Definition 5.4. A homogeneous network is a coupled cell network such that $B(c, d)$ $\neq \emptyset$ for every pair of cells $c, d$. A homogeneous network that has one equivalence class of edges is said to have identical coupling. The valence of an identical coupling network is the number of arrows in any (and hence every) input set.
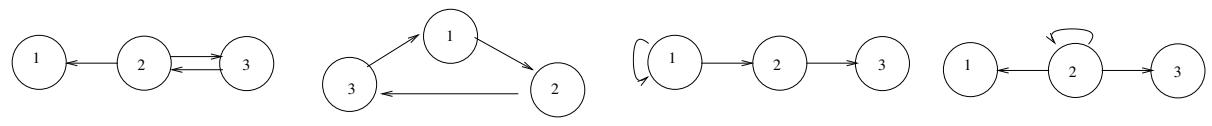

Figure 21. The four valence one identical coupling connected 3 -cell networks.

There are four connected 3-cell networks with identical coupling and valence 1 (see Figure 21) and 34 such networks with valence 2 (see Leite [55, 56] and Figure 221). It is possible for two networks to generate the same systems of differential equations [18, 19, 42. Such redundancies have been eliminated in this enumeration. The number of identical coupling networks grows exponentially with the number of cells. For example, there are precisely 13,505,066,262,007 connected 6 -cell networks with identical coupling and valence 6 (Aldosray and Stewart [1]).

Motifs. Many applications are modeled by large networks; these include gene transcriptional networks, networks of neurons, electronic circuits, and the World Wide Web, to name just a few. Recent work on classes of networks has shown that certain small subnets appear more frequently in applications than they would in random networks, and the frequently appearing subnets have been called motifs [59, 65. Typical motifs include the feedforward loop, the three chain, and the uplinked mutual dyad; these subnets are pictured in Figure 23. Note the similarities with Figure 21 (3) and Figure 22 $(23,33)$. Some typical dynamics of the network in Figure 21 (3) is discussed in Example 11.1, it is shown there that near certain bifurcations this network acts to amplify signals down the chain.

\section{Admissible vector fields}

We now define the class $\mathcal{F}_{G}^{P}$ of 'admissible' vector fields corresponding to a given coupled cell network $G$. This class consists of all vector fields that are compatible with the labeled graph structure or equivalently are symmetric under the groupoid $\mathcal{B}_{G}$. The class $\mathcal{F}_{G}^{P}$ also depends on a choice of 'total phase space' $P$, which we assume is fixed throughout the subsequent discussion. We construct $P$ as follows. 

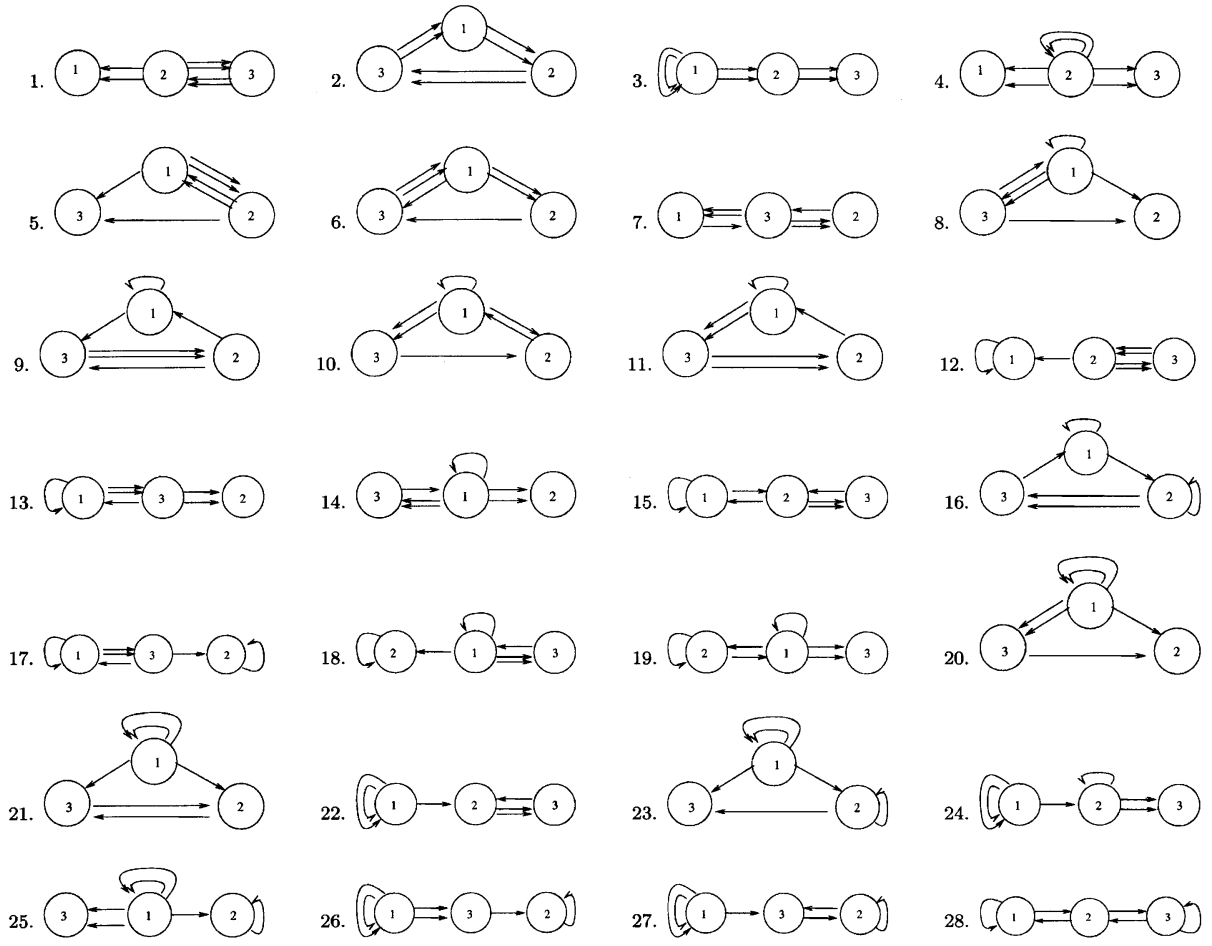

27. (C) $(1)-(3)=2)$
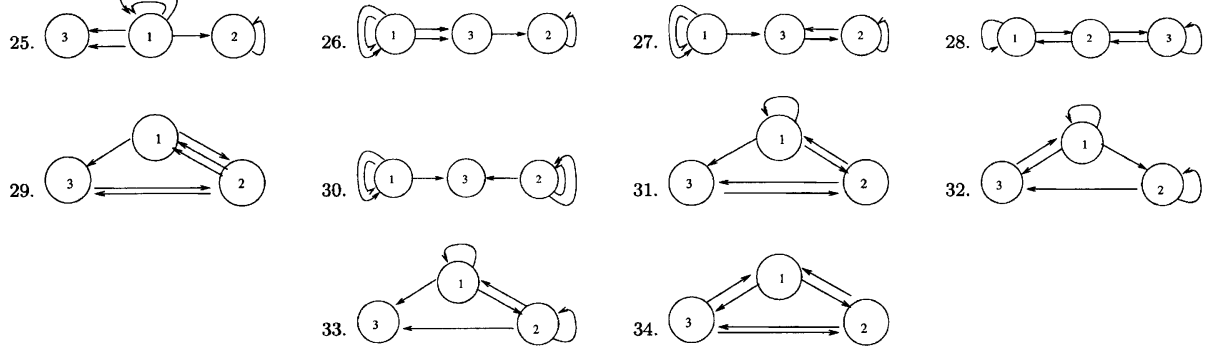

Figure 22. Connected 3-cell networks with identical coupling and valence 2 , from [55, 56].
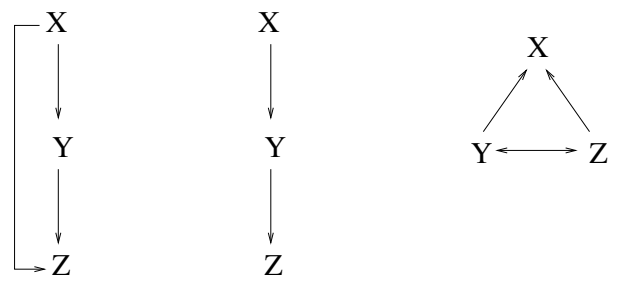

Figure 23. Typical motifs: feedforward loop, three chain, and uplinked mutual dyad [59].

For each cell in $\mathcal{C}$ define a cell phase space $P_{c}$. This must be a smooth manifold of dimension $\geq 1$, which for simplicity we assume is a nonzero finite-dimensional real vector space. We require

$$
c \sim_{C} d \quad \Longrightarrow \quad P_{c}=P_{d}
$$


and we employ the same coordinate systems on $P_{c}$ and $P_{d}$. Only these identifications of cell phase spaces are canonical; that is, the relation $c \sim_{C} d$ implies that cells $c$ and $d$ have the same phase space, but not that they have isomorphic (conjugate) dynamics.

Define the corresponding total phase space to be

$$
P=\prod_{c \in \mathcal{C}} P_{c}
$$

and employ the coordinate system

$$
x=\left(x_{c}\right)_{c \in \mathcal{C}}
$$

on $P$.

More generally, suppose that $\mathcal{D}=\left(d_{1}, \ldots, d_{s}\right)$ is any finite ordered subset of $s$ cells in $\mathcal{C}$. In particular, the same cell can appear more than once in $\mathcal{D}$. Define

$$
P_{\mathcal{D}}=P_{d_{1}} \times \cdots \times P_{d_{s}}
$$

and write

$$
x_{\mathcal{D}}=\left(x_{d_{1}}, \ldots, x_{d_{s}}\right)
$$

where $x_{d_{j}} \in P_{d_{j}}$. For a given cell $c$ the internal phase space is $P_{c}$ and the coupling phase space is

$$
P_{\mathcal{T}(I(c))}=P_{\mathcal{T}\left(i_{1}\right)} \times \cdots \times P_{\mathcal{T}\left(i_{s}\right)}
$$

where $\mathcal{T}(I(c))$ denotes the ordered set of cells $\left(\mathcal{T}\left(i_{1}\right), \ldots, \mathcal{T}\left(i_{s}\right)\right)$ as the arrows $i_{k}$ run through $I(c)$.

Suppose $c, d \in \mathcal{C}$ and $c \sim_{I} d$. For any $\beta \in B(c, d)$, define the pullback map

$$
\beta^{*}: P_{\mathcal{T}(I(d))} \rightarrow P_{\mathcal{T}(I(c))}
$$

by

$$
\left(\beta^{*} z\right)_{\mathcal{T}(i)}=z_{\mathcal{T}(\beta(i))}
$$

for all $i \in I(c)$ and $z \in P_{\mathcal{T}(I(d))}$. We use pullback maps to relate different components of a vector field associated with a given coupled cell network. Specifically, the class of vector fields that is encoded by a coupled cell network is given by:

Definition 6.1. A vector field $f: P \rightarrow P$ is $G$-admissible if:

(a) (domain condition) For all $c \in \mathcal{C}$ the component $f_{c}(x)$ depends only on the internal phase space variables $x_{c}$ and the coupling phase space variables $x_{\mathcal{T}(I(c))}$; that is, there exists $\hat{f}_{c}: P_{c} \times P_{\mathcal{T}(I(c))} \rightarrow P_{c}$ such that

$$
f_{c}(x)=\hat{f}_{c}\left(x_{c}, x_{\mathcal{T}(I(c))}\right)
$$

(b) (pullback condition) For all $c, d \in \mathcal{C}$ and $\beta \in B(c, d)$

$$
\hat{f}_{d}\left(x_{d}, x_{\mathcal{T}(I(d))}\right)=\hat{f}_{c}\left(x_{d}, \beta^{*} x_{\mathcal{T}(I(d))}\right)
$$

for all $x \in P$.

The pullback condition (b) is a natural generalization of the usual equivariance condition for a group action. The domain condition (a) is necessary for (b) to make sense, and this type of constraint has not been formalized in the group-theoretic literature. The interplay between these two conditions is what lends the groupoid formalism its own special flavor.

It follows that $f$ is determined if we specify one mapping (on the appropriate spaces) for each input equivalence class of cells. Indeed, each admissible vector 
field on a homogeneous cell system is uniquely determined by a single mapping $f_{c}$ at some (any) node $c$. In general, each component $f_{c}$ of $f$ is invariant under the vertex group $B(c, c)$. Indeed, every such invariant function determines a unique admissible vector field via pullback to the other components.

An ODE defined by an admissible vector field on a coupled cell network is called a coupled cell system. It is now easy to verify that the ODEs previously associated with specific networks, on heuristic grounds, are precisely the admissible ODEs in the formal sense.

Larger classes of coupled cell systems can be studied by considering vector fields that commute with only a subgroupoid of $\mathcal{B}_{G}$. The basic theorems that we describe are valid in this context as well [46].

\section{BALANCED EQUIVALENCE RELATIONS}

With the formalities in place, we can now seek a necessary and sufficient condition for a pattern of synchrony to be possible for all admissible ODEs. It turns out that the pattern must be 'balanced', which roughly speaking means that synchronous cells must have synchronous input sets. This condition is obviously sufficient; its necessity is slightly more subtle.

We then apply the result to dynamics on planar lattices (an ODE version of 'coupled map lattices', currently popular in the physics literature because of connections to statistical mechanics). In particular we observe that lattices with short-range connectivity can support states with unexpected properties, whereas longer-range connectivity implies that all synchronous patterns are doubly-periodic.

We have seen that Example 4.4 exhibits patterns of synchrony that correspond to a particular kind of flow-invariant subspace, namely, one that is invariant under all admissible vector fields. Such subspaces are said to be robust and are known either as polydiagonals or polysynchronous subspaces. A basic theorem, Theorem 7.2 below, characterizes all robust polydiagonals in combinatorial terms.

The key feature of a robust polydiagonal is that identifying synchronous variables leads to a consistent system of ODEs. We emphasized this feature when discussing the two examples, and it leads fairly directly to the concept of 'balance'.

In a formal treatment, we describe a pattern of synchrony in terms of an equivalence relation $\bowtie$ on the set $\mathcal{C}$ of all cells. An equivalence relation $\bowtie$ on $\mathcal{C}$ determines a unique partition of $\mathcal{C}$, in which the 'parts' are the $\bowtie$-equivalence classes. Conversely, any partition determines a unique equivalence relation. The corresponding polysynchronous subspace is defined by

$$
\Delta_{\bowtie}=\left\{x \in P: c \bowtie d \Longrightarrow x_{c}=x_{d}\right\} .
$$

For instance, in Example 4.4 the partition $\{\{1,3\},\{2,4\},\{5\}\}$ has polysynchronous subspace $\{(x, y, x, y, z)\}$. Entries $x_{c}$ with $\bowtie$-equivalent $c$ 's (that is, with $c$ 's in the same part of the partition) are equal.

An equivalence $\bowtie$ induces an identification of variables $x_{c}, x_{d}$ whenever $c \bowtie d$. By (7.1) this identification has the same effect as restricting the vector field $f$ to

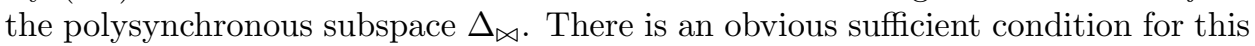
restriction to leave $\Delta_{\bowtie}$ flow-invariant under $f \in \mathcal{F}_{G}^{P}$, namely:

$$
c \bowtie d \Longrightarrow f_{c}=f_{d} \text { on } \Delta_{\bowtie} .
$$

We can reformulate this condition in terms of input isomorphisms, leading to the following concept: 
Definition 7.1. An equivalence relation $\bowtie$ on $\mathcal{C}$ is balanced if for every $c, d \in \mathcal{C}$ with $c \bowtie d$, there exists an input isomorphism $\beta \in B(c, d)$ such that $\mathcal{T}(i) \bowtie \mathcal{T}(\beta(i))$ for all $i \in I(c)$.

It is easy to see that condition (7.2) is valid if and only if $\bowtie$ is balanced. This condition does not depend on $f$.

There is a graphical way to test whether a given equivalence relation $\bowtie$ is balanced. Color the cells in $\mathcal{C}$ so that two cells have the same color precisely when they are in the same $\bowtie$-equivalence class. Color the tail of each arrow by using the color of the corresponding cell. Say that an input-isomorphism $\beta: I(c) \rightarrow I(d)$ is color-preserving if $\mathcal{T}(i)$ and $\mathcal{T}(\beta(i))$ have the same color for all $i \in I(c)$. Then $\bowtie$ is balanced if and only if: If $c, d$ have the same color, then there exists a colorpreserving input isomorphism $\beta: I(c) \rightarrow I(d)$.

In particular, $B(c, d) \neq \emptyset$, so $c \sim_{I} d$. Therefore any balanced equivalence $\bowtie$ refines $\sim_{I}$, meaning that

$$
c \bowtie d \Longrightarrow c \sim_{I} d .
$$

In fact, being balanced is equivalent to defining a robust polysynchronous subspace:

Theorem 7.2. Let $\bowtie$ be an equivalence relation on a coupled cell network. Then $\bowtie$ is robustly polysynchronous if and only if $\bowtie$ is balanced.

The proof, given in [42, 72], can be summarized as follows. By constructing appropriate linear admissible vector fields, we can show that if a polysynchronous subspace is flow-invariant under all linear admissible vector fields, then the associated equivalence $\bowtie$ must be balanced. The reduction process tells us that when $\bowtie$ is balanced, the corresponding polysynchronous subspace $\Delta_{\bowtie}$ is flow-invariant under all admissible vector fields (linear or not). Clearly, flow-invariance under all admissible vector fields implies flow-invariance under all linear admissible vector fields.

The combinatorial notion of balanced relations enables us to find patterns of synchrony. We use square lattice dynamical systems as an example. This example also illustrates the importance of network architecture (as opposed to symmetry) in the determination of patterns of synchrony. Let the planar integer square lattice be the index set for a coupled cell network and assume identical nearest neighbor coupling, so each cell has four nearest neighbors. See Figure 24 (left).
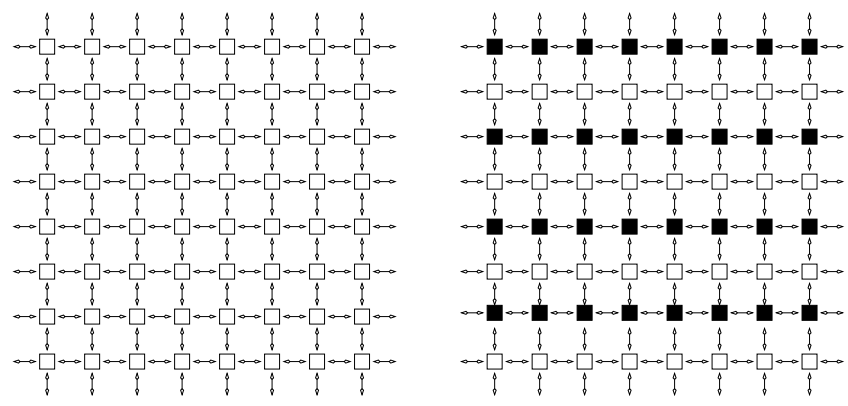

Figure 24. Square lattice network with nearest neighbor coupling. Balanced 2-coloring on right. 
It is straightforward to show (using either symmetry or balanced relations) that the pattern of synchrony pictured in Figure 24 corresponds to a flow-invariant subspace. In this correspondence all white cells are synchronous and all black cells are synchronous. It is observed in 32] that interchanging black and white cells on any upward sloping diagonal leads to another balanced 2-coloring and hence to a continuum of balanced 2-colorings and patterns of synchrony. Some examples are shown in Figure 25.
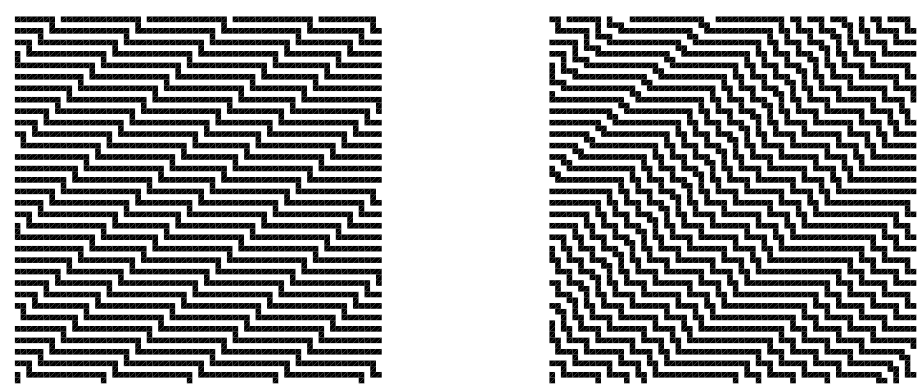

Figure 25. Two examples of balanced 2-coloring of square lattice network obtained from Figure 24 (right) by diagonal shifts.

Wang [81] has classified all balanced 2-colorings for planar square and hexagonal lattices with nearest neighbor coupling. This classification shows that up to symmetry there is only a finite number of balanced 2-colorings with both nearest and next nearest neighbor coupling and that all of the corresponding patterns of synchrony are spatially periodic. These facts are clearly false for lattice dynamical systems with just nearest neighbor coupling. In fact, with nearest and next nearest neighbor coupling it can be shown that for each $k$ there is a finite number of balanced $k$-colorings, and all of these are spatially periodic [3].

\section{RIGID EQUILIBRIA}

It is easy to construct examples of synchronized states that do not correspond to balanced equivalence relations, but all such examples known to us are 'fragile', that is, destroyed by small admissible perturbations of the vector field. We can prove this fragility when the states are hyperbolic equilibria - a fact that has some interest because it does not start with the assumption that an entire subspace must be flow-invariant for all admissible vector fields. Nevertheless, we deduce the presence of such a subspace. So observed properties of a single (equilibrium) state can imply regularities of the entire network architecture.

We conjecture that similar results apply to any synchronized state lying on a hyperbolic attractor (perhaps periodic, or even chaotic), but this seems difficult to prove. We discuss this problem in Section 10.

To set the scene, recall Theorem 7.2 which proves that the balanced equivalence relations on a given network classify its robust patterns of synchrony - that is, those patterns that are determined by flow-invariant subspaces for all admissible vector fields. Robustness is a strong requirement, and it might seem that something weaker might apply to specific synchronized dynamical states, such as equilibria, periodic states, or even synchronized chaos. 
The main result of this section is that the only 'rigid' patterns of synchrony for hyperbolic equilibria are those determined by balanced equivalence relations. Rigidity here means that the pattern does not change when the vector field is perturbed by a sufficiently small admissible perturbation. The proof introduces some useful general methods.

Let $x_{0}=\left(x_{1}^{0}, \ldots, x_{N}^{0}\right) \in P$. Define the equivalence relation $\equiv_{x_{0}}$ by $c \equiv_{x_{0}} d$ if and only if $c \sim_{C} d$ and $x_{c}^{0}=x_{d}^{0}$. Suppose that we color two cells $c$ and $d$ the same color if and only if $c \equiv_{x_{0}} d$. Then this coloring is the pattern of synchrony associated to $x_{0}$. Note that

$$
\Delta_{\equiv_{x_{0}}}=\left\{x \in P: x_{c}=x_{d} \quad \text { if } \quad c \equiv_{x_{0}} d\right\}
$$

is the smallest subspace of $P$ that contains all points with the same pattern of synchrony as $x_{0}$.

Definition 8.1. Let $x_{0} \in P$ be a hyperbolic equilibrium of a $C^{1}$ admissible cell system. The equivalence relation $\equiv_{x_{0}}$ is rigid if in each $C^{1}$ perturbed admissible system the unique hyperbolic equilibrium near $x_{0}$ remains in $\Delta_{\equiv_{x_{0}}}$. We also say that the pattern of synchrony defined by $x_{0}$ is rigid.

Theorem $8.2([42])$. The equivalence relation $\equiv_{x_{0}}$ determined by the hyperbolic equilibrium $x_{0}$ is rigid if and only if $\equiv_{x_{0}}$ is balanced.

The proof uses the concept of a strongly admissible diffeomorphism:

Definition 8.3. A map $G: P \rightarrow P$ is strongly admissible if $G_{c}(x)=G_{c}\left(x_{c}\right)$ for every cell $c$ and $G_{c}=G_{d}$ for every pair of cells where $c \sim_{C} d$.

A strongly admissible map $G$ is admissible, since $c \sim_{I} d$ implies that $c \sim_{C} d$ and hence $G_{c}=G_{d}$. The key property of strongly admissible maps is:

Lemma 8.4. Let $F: P \rightarrow P$ be admissible and let $G: P \rightarrow P$ be strongly admissible. Then $F \circ G$ and $G \circ F$ are admissible.

Example 8.5. These composition properties fail for merely admissible maps, even in the linear case. These failures are a significant departure from the groupsymmetric case. Examples of failure are easy to come by. Figure 18 above is perhaps the simplest. It has two cells and is homogeneous with valence 2 . The admissible linear maps are those of the form

$$
L=\left[\begin{array}{cc}
A & 2 B \\
A+B & B
\end{array}\right]
$$

The product of two such maps is

$$
\left[\begin{array}{cc}
A & 2 B \\
A+B & B
\end{array}\right]\left[\begin{array}{cc}
C & 2 D \\
C+D & D
\end{array}\right]=\left[\begin{array}{cc}
A C+2 B C+2 B D & 2 A D+2 B D \\
A C+2 B C+B D & 2 A D+3 B D
\end{array}\right]
$$

which in general is not of the same form.

\section{QUOTIENT NETWORKS}

The story so far has focused on the notion of a balanced equivalence relation $\bowtie$ on a network $G$ and the existence of associated synchronized states in a robust or rigid manner. But we can also ask: what kinds of synchronous dynamics can occur for a given network and a given balanced equivalence relation? The answer is striking: the dynamics is determined by a network whose nodes correspond to 
clusters of synchronous cells (that is, $\bowtie$-equivalence classes) and whose edges are defined to preserve the 'colored input sets' defined by $\bowtie$. We call this network the quotient of $G$ by $\bowtie$ (because it is obtained by identifying cells in the same $\bowtie$-equivalence class). In this section we introduce quotient networks, remark that they necessarily may have multiple edges or connections from a cell to itself, and prove that any dynamic on the quotient 'lifts' to a synchronous dynamic on the original network.

In Example 4.4 we saw that the restricted equations for a particular pattern of synchrony (a partition with three parts) are precisely the admissible equations for a smaller network, which in that case had three cells and $\mathbf{S}_{3}$ symmetry. The existence of such a network is a general phenomenon. In this section we observe that each balanced equivalence relation $\bowtie$ of a coupled cell network $G$ induces a unique canonical coupled cell network $G_{\bowtie}$ on $\Delta_{\bowtie}$, called the quotient network. (We use the term 'quotient' because on the graph-theoretic level - network topology - it is obtained by identifying members of equivalence classes.) This is not the case in the setting of 72 , where quotient networks always exist but uniqueness fails. It was also shown in [72, in the context of coupled cell systems without self-coupling and multiple arrows, that every admissible vector field on the original network restricts to an admissible vector field on $\Delta_{\bowtie}$ in every quotient network. However, in general an admissible vector field on a quotient network cannot be extended (or 'lifted') to an admissible vector field on the original network. A full discussion, with necessary and sufficient conditions for such an extension to exist, can be found in Dias and Stewart [18. It is the uniqueness and lifting properties that constitute the above-mentioned 'technical advantages' of the multiarrow formalism.

In the present context, then, it can be proved that admissible vector fields restrict to admissible vector fields and every admissible vector field on the canonical quotient $G_{\bowtie}$ lifts to an admissible vector field on $G$. We begin by defining the (canonical) quotient network.

To define a network (see Definition 5.1) we must specify (a) the cells, (b) an equivalence relation on cells, (c) the arrows, (d) an equivalence relation on arrows, and (e) the head and tail incidence relations. We must also (f) prove a consistency relation between arrows and cells. We do each of these in turn.

(a) Let $\bar{c}$ denote the $\bowtie$-equivalence class of $c \in \mathcal{C}$. The cells in $\mathcal{C}_{\bowtie}$ are the $\bowtie$-equivalence classes in $\mathcal{C}$, that is,

$$
\mathcal{C}_{\bowtie}=\{\bar{c}: c \in \mathcal{C}\} .
$$

Thus we obtain $\mathcal{C}_{\bowtie}$ by forming the quotient of $\mathcal{C}$ by $\bowtie$, that is, $\mathcal{C}_{\bowtie}=\mathcal{C} / \bowtie$.

(b) Define

$$
\bar{c} \sim_{C_{\bowtie}} \bar{d} \Longleftrightarrow c \sim_{C} d .
$$

The relation $\sim_{C_{\triangleright}}$ is well-defined since $\bowtie$ refines $\sim_{C}$.

(c) Let $\mathcal{S} \subset \mathcal{C}$ be a set of cells consisting of precisely one cell $c$ from each $\bowtie$ equivalence class. The input arrows for a quotient cell $\bar{c}$ are identified with the input arrows in cell $c$, where $c \in \mathcal{S}$, that is, $I(\bar{c})=I(c)$.

When viewing the arrow $i \in I(c)$ as an arrow in $I(\bar{c})$, we denote that arrow by $\bar{i}$. Thus, the arrows in the quotient network are the projection of arrows in the original network formed by the disjoint union

$$
\mathcal{E}_{\bowtie}=\bigcup_{c \in \mathcal{S}} I(c)
$$


The definition of the quotient network structure is independent of the choice of the representative cells $c \in \mathcal{S}$.

(d) Two quotient arrows are equivalent when the original arrows are equivalent. That is,

$$
\overline{i_{1}} \sim_{E_{\bowtie}} \overline{i_{2}} \quad \Longleftrightarrow \quad i_{1} \sim_{E} i_{2}
$$

where $i_{1} \in I\left(c_{1}\right), i_{2} \in I\left(c_{2}\right)$, and $c_{1}, c_{2} \in \mathcal{S}$.

(e) Define the heads and tails of quotient arrows by

$$
\mathcal{H}(\bar{i})=\overline{\mathcal{H}(i)} \quad \mathcal{T}(\bar{i})=\overline{\mathcal{T}(i)} .
$$

(f) It is easy to verify that the quotient network satisfies the consistency condition in Definition 5.1(f). The quotient network is independent of the choice of cells in $\mathcal{S}$ because $\bowtie$ is balanced.

Remark 9.1. It is straightforward that quotients of homogeneous networks are homogeneous and that quotients of identical coupling networks are identical coupling.

Theorem $9.2([42])$. Let $\bowtie$ be a balanced equivalence relation on a coupled cell network $G$.

(a) The restriction of a $G$-admissible vector field to $\Delta_{\bowtie}$ is $G_{\bowtie}$-admissible.

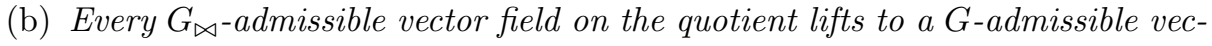
tor field on the original network.

Remark 9.3. Example 4.4 illustrates that identical coupling networks without self-coupling or multiple arrows can have quotient networks with these features. In 42. we show that every identical coupling network that has self-coupling and/or multiple arrows lifts to an identical coupling network without these features, in the sense that the original network is a quotient network of the larger network. An example of such a lifting is given in Figure 26.
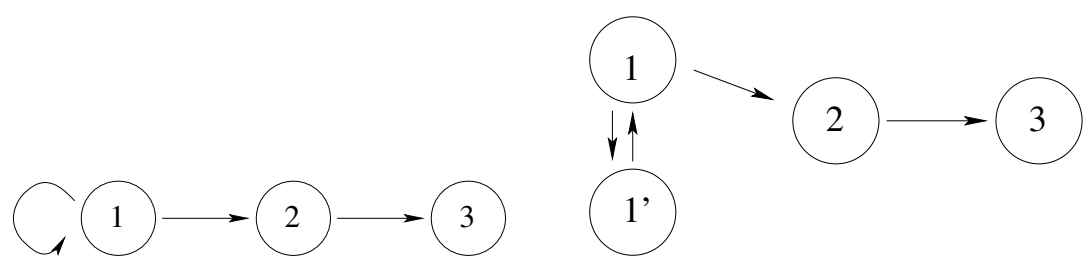

Figure 26. (Left) Three-cell feed-forward network with selfcoupling. (Right) A lift to a four-cell network with identical coupling.

In fact, this statement is true for all networks - not just identical coupling networks. The proof is similar to that in 42, but some care must be taken to ensure that the inductive process stops.

This fact provides yet another reason to believe that the natural category of networks to study is the one that permits self-coupling and multiple arrows. This gives added importance to the study of the dynamical properties of the 3-cell networks shown in Figures 21 and 22 . 
Moreover, this observation/conjecture has implications for modelling. Often complicated networks are simplified by observing that groups of cells behave synchronously and can be lumped together as a single unit. When this kind of simplification is made, our abstract results show that self-coupling and multiple arrows can result, which implies that there may exist constraints on the 'lumped' system, which must be accounted for when analyzing its behavior.

\section{RIGID PERIODIC STATES}

There are reasons to believe that Theorem 8.2 has an analog for periodic states, with a generalization to phase-relationships and a remarkable consequence. As we write, however, the periodic theory depends upon an unproved but plausible conjecture, which we call the Rigid Phase Conjecture. We outline the motivation for this conjecture, some of the evidence in its favor, and its main consequence, which is: in any network, any nontrivial rigid pattern of phase shifts always derives from a cyclic-group symmetry of a closely related network.

To motivate the discussion, consider the animal locomotion network of Figure 10 . We know that this network supports a variety of phase-related states, in which particular pairs of cells $c, d$ have the same waveform, possibly subject to specific phase shifts.

For example, consider the 'walk' gait. Here there is a single periodic waveform, call it $X(t)$, such that

$$
\begin{aligned}
& x_{1}(t)=x_{6}(t)=X(t) \\
& x_{3}(t)=x_{8}(t)=X(t-T / 4) \\
& x_{5}(t)=x_{2}(t)=X(t-2 T / 4) \\
& x_{7}(t)=x_{4}(t)=X(t-3 T / 4)
\end{aligned}
$$

where $T$ is the period. Using symmetry, it is not hard to prove that provided the periodic cycle is hyperbolic in the sense of Katok and Hasselblatt [50], chapter 6, section 2, the same pattern of phase relations arises if the vector field is perturbed slightly (while preserving the symmetry). Hyperbolicity implies that there is a unique perturbed periodic cycle close to the original one and any change of symmetry would violate uniqueness.

Simple examples show that in general networks, hyperbolicity alone is not sufficient to guarantee that phase relations are preserved by small perturbations of the vector field. So it seems reasonable to attempt to characterize cases where the phase relations persist under perturbation. By analogy with the case of equilibria, we will call such periodic states 'rigid'.

Using balanced equivalence relations and quotients we can easily construct coupled cell networks that have trivial symmetry but also support rigid phase-related periodic states by lifting from a quotient with cyclic-group symmetry. In this construction, the equivalence relation 'same phase' (or 'synchronous') is balanced. The traveling wave in a feed-forward network is an example of precisely this construction.

The existence of such networks lies behind the question raised in Section 3 in connection with the 8-cell network for animal locomotion, namely: might a simpler, asymmetric network perform the same task for groupoid-related reasons? The Rigid Phase Conjecture, if true, should resolve this issue. Roughly speaking, we expect the 8-cell network to be a quotient of any network that exhibits the necessary 
range of phase relations, implying that no smaller network is suitable. However, we have not yet worked out the details of this idea, and it may require some technical hypotheses (perhaps including bilateral symmetry).

We now explore the conditions under which a hyperbolic periodic state can have rigid phase relationships.

Definition 10.1. Let $\mathbf{X}=\{x(t)\}$ be a periodic orbit of a $G$-admissible vector field $f$. Assume that $x(t)$ is hyperbolic. Therefore if $\hat{f}$ is a sufficiently small perturbation of $f$, then the vector field $\hat{f}$ has a unique periodic orbit $\hat{\mathbf{X}}=\{\hat{x}(t)\}$ near $x(t)$ having period $\hat{T}$ near $T$ (Hirsch et al. [48). We call $\hat{\mathbf{X}}$ the perturbed periodic orbit. Then $\mathbf{X}$ is rigid if, in addition to hyperbolicity, any phase relation

$$
x_{c}(t) \equiv x_{d}(t+\theta)
$$

is preserved in the corresponding perturbed periodic orbit:

$$
\hat{x}_{c}(t) \equiv \hat{x}_{d}(t+\hat{\theta})
$$

where $\hat{\theta} / \hat{T}=\theta / T$. That is, the phase shift represents the same proportion of the period.

The Rigid Phase Conjecture. Using strongly admissible coordinate changes it is possible to prove:

Theorem 10.2 (Rigid Input Theorem). For rigid $\mathbf{X}=\{x(t)\}$, each phase relation of the form (10.2) implies that $c \sim_{I} d$.

Now we can play (10.2) off against the pullback condition. For the appropriate $f \in \mathcal{F}_{G}^{P}$ we have:

$$
\begin{aligned}
f_{c}(x(t)) & \equiv \dot{x}_{c}(t) \\
& \equiv \dot{x}_{d}(t+\theta) \\
& \equiv f_{d}(x(t+\theta)) \\
& \equiv f_{c}\left(\beta^{*} x(t+\theta)\right)
\end{aligned}
$$

where by the Rigid Input Theorem, $\beta \in B(c, d) \neq \emptyset$. Therefore we obtain

$$
f_{c}(x(t)) \equiv f_{c}\left(\beta^{*} x(t+\theta)\right) .
$$

We are thus led to:

Conjecture 10.3 (Rigid Phase Conjecture). Suppose that $\mathbf{X}$ is rigid. If $c, d$ satisfy (10.2), then there exists $\beta \in B(c, d)$ such that

$$
x_{I(c)}(t) \equiv \beta^{*} x_{I(d)}(t+\theta) .
$$

Intuitively: 'Rigid phase relations are inherited by input sets, up to input isomorphism.'

The primary motivation for this conjecture is that (10.4) is sufficient for (10.3) to hold, and it is difficult to think of any other reason for such a relationship that could survive perturbation of $f$. We can prove special cases of the conjecture under various technical hypotheses (Stewart and Parker [73, 74]) or for particular small networks, but a complete proof has not yet been found. These proofs employ a technique that is of interest in its own right: 'symmetrizing over the groupoid', analogous to the well-known method of summing (or integrating) over a group action. The main obstacle is to obtain enough control over how the periodic cycle moves when the vector field is perturbed, bearing in mind that the perturbation must be $G$-admissible. 
One piece of evidence for the Rigid Phase Conjecture is that it implies a periodic analog of Theorem 8.2 .

Theorem 10.4. With the above notation, the Rigid Phase Conjecture implies that the relation of synchrony defined by

$$
c \bowtie d \Longleftrightarrow x_{c}(t) \equiv x_{d}(t)
$$

is balanced.

To prove this, set $\theta=0$ in (10.4).

The Rigid Phase Conjecture has more to offer: it allows us to characterize rigid patterns of synchrony. Subject to some technical assumptions which we ignore here, they always arise by lifting from a quotient network that has cyclic group symmetry.

Certainly, the existence of such a quotient is sufficient for certain rigid phase patterns to occur. For example, we know that the coupled cell network of Figure 13 supports a discrete rotating wave - a solution $x(t)$ with phase shifts $0, T / 3,2 T / 3$, where $T$ is the period. Such a state can be lifted to any network that has the 3 -cell ring as a quotient - in fact, this is what we did to generate the traveling wave in the 7-cell feed-forward network.

Discrete rotating waves are typical of any network with cyclic-group symmetry, even when the network is not homogeneous. The next example illustrates circumstances in which nontrivial phase shifts can occur without cyclic-group symmetry but for trivial (and avoidable) reasons, which we therefore define away.

Example 10.5. Figure 27 shows a network in which a ring of three cells drives two other cells. If cell 0 forces cell 3 and cell 1 forces cell 4 in the same manner, with a 1:1 resonance between cells 0,3 (hence also between 1,4), we can arrange a $T$-periodic state with

$$
\begin{aligned}
& x_{0}(t)=x_{1}(t+T / 3)=x_{2}(t+2 T / 3) \\
& x_{3}(t)=x_{4}(t+T / 3) .
\end{aligned}
$$

If a sixth cell (cell 5) were added, the network would have $\mathbf{Z}_{3}$ symmetry. However, with cell 5 missing, no such symmetry group occurs, but cells 3 and 4 are rigidly phase-locked with phase-shift $T / 3$ by equivariant dynamics applied to the 6 -cell system.

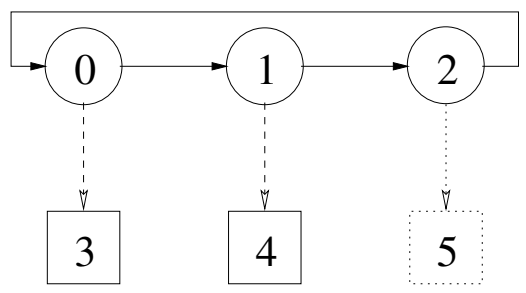

FiguRE 27. A three-cell ring with dangling cells.

We can avoid such examples by assuming that $G$ is path-connected, meaning that there is a directed path from any cell to any other cell. The Rigid Phase Conjecture implies (Stewart and Parker [75]) the following characterization of rigid phase patterns in the path-connected case: 
Theorem 10.6. Assume the Rigid Phase Conjecture. Suppose that $G$ is pathconnected, and $B(c, c)=\mathbf{1}$ for all $c \in \mathcal{C}$. Let $\mathbf{X}=\{x(t)\}$ be a rigid phase-related periodic state of an admissible vector field $f$. Let $\bowtie$ be the relation of synchrony on $\mathbf{X}$, which is balanced (by the Rigid Phase Conjecture). Then the quotient network $G / \bowtie$ supports a faithful $\mathbf{Z}_{a}$-action, for some integer $a \leq|G / \bowtie|$, and $x(t)$ is a discrete rotating wave whose orbit is fixed setwise by $\mathbf{Z}_{a}$.

Corollary 10.7. With the above assumptions, let $|\mathcal{C}|=N$. Then the phase shifts of any rigid phase pattern on $G$ are integer multiples of $T / a$, where $1 \leq a \leq N$.

\section{SyNChrony-BREAKING BIFURCATIONS}

Dynamical systems theory involves (at least) two major areas. One considers the different types of dynamical states that a system can exhibit. The other, bifurcation theory, analyzes the typical transitions between states as parameters are varied.

The discussion until now has taken place in the first area: individual states of the system. Now we turn attention to bifurcations. Once more, we motivate the network theory by recalling what is known about bifurcations of symmetric systems.

Symmetry-breaking bifurcations have proved useful in a number of applications [37, 41. The usual setup is the loss of stability, as a parameter $\lambda$ is varied, of a $\Gamma$-invariant equilibrium $x_{0}$ in a $\Gamma$-equivariant system of differential equations $\dot{x}=f(x, \lambda)$. Without loss of generality we may assume that bifurcations are located at $\lambda=0$ and occur when the Jacobian matrix $J=(d f)_{x_{0}, 0}$ has eigenvalues on the imaginary axis. The equivariant bifurcation theory that we describe here is discussed in detail in [37, 41.

The generic theory goes as follows. There are two classes of bifurcations: steadystate and Hopf. Steady-state bifurcation occurs when the critical eigenvalues are 0, and Hopf bifurcation occurs when the critical eigenvalues are nonzero and purely imaginary (by scaling time we can assume that the critical eigenvalues are at $\pm i$ ). Real representation theory tells us that the commuting linear maps of an irreducible representation form a skew-field and so are isomorphic either to the reals (absolutely irreducible) or the complexes or quaternions (nonabsolutely irreducible). It can be shown that generically the center subspace $E^{c}$ of $J$ is an absolutely irreducible representation of $\Gamma$ at steady-state bifurcations and a $\Gamma$-simple representation at Hopf bifurcations, where a representation is $\Gamma$-simple if it is either the direct sum of two isomorphic absolutely irreducible representations or a nonabsolutely irreducible subspace.

In equivariant theory, the Equivariant Branching Lemma states that generically each axial subgroup of $\Gamma$ leads to a branch of equilibria at steady-state bifurcations. Similarly the Equivariant Hopf Theorem states that each $\mathbf{C}$-axial subgroup of $\Gamma \times \mathbf{S}^{1}$ leads to a branch of periodic states at Hopf bifurcations. A subgroup $\Sigma \subset \Gamma$ is axial if $\Sigma$ is an isotropy subgroup of the action of $\Gamma$ on $E^{c}$ and $\operatorname{dim} \operatorname{Fix}(\Sigma)=1$ in this action. In Hopf bifurcation the action of $\mathbf{S}^{1}$ on $E^{c}$ is given by $e^{t J}$, which commutes with the action of $\Gamma$ on $E^{c}$. A subgroup $\Sigma \subset \Gamma \times \mathbf{S}^{1}$ is $\mathbf{C}$-axial is $\Sigma$ is an isotropy subgroup of the action of $\Gamma \times \mathbf{S}^{1}$ on $E^{c}$ and $\operatorname{dim} \operatorname{Fix}(\Sigma)=2$ in this action.

For example, $\Gamma$-simple representations of abelian groups are all two-dimensional and the Equivariant Hopf Theorem reduces to the standard Hopf theorem in this case, with the addition that the bifurcating periodic solutions have spatio-temporal symmetries $H=\Gamma$ and space symmetries $K$ equal to the kernel of the representation of $\Gamma$ on $E^{c}$ (using the terminology of Section 2). It follows that each of the primary 
quadrupedal gaits described in Section 3 can in principle be obtained by Hopf bifurcation from a $\mathbf{Z}_{4} \times \mathbf{Z}_{2}$-invariant equilibrium (see [37, 38]). On the other hand, perhaps surprisingly, the multirhythm solution illustrated in Figure 6 cannot be obtained by such a Hopf bifurcation [32.

The question that we would like to address is whether there is an analogous theory of synchrony-breaking bifurcations from a synchronous equilibrium. So far the answer is 'no', since there does not appear to be a groupoid representation theory that is suited to the task. Nevertheless, some unusual examples indicate that such a theory will lead to new phenomenology, but these examples also indicate why a general theory may be difficult to develop.

We assume that a coupled cell system has a synchronous equilibrium and that the Jacobian $J$ at the synchronous equilibrium has a nonzero center subspace $E^{c}$. As indicated above, generic codimension one equivariant bifurcations occur with the Jordan normal form of $J \mid E^{c}$ being trivial. It is only in codimension two that Takens-Bogdanov type singularities with nilpotent normal forms occur. This is not the case in coupled cell systems, and we present two examples here.

We begin by recalling that in ordinary generic Hopf bifurcation there is a unique branch of periodic solutions that emanates from the origin and that the amplitude of these periodic states grows at a rate of $\lambda^{1 / 2}$. We now discuss three cases where network architecture forces multiple branches of periodic solutions and, in one case, a huge growth of $\lambda^{1 / 6}$ in the amplitude of bifurcating periodic states. After presenting the three examples we will discuss briefly how the proof proceeds, as there is one common feature to all three examples - codimension one bifurcations lead to a nilpotent Jordan normal form. The details of this analysis may be found in [24, 32].

Example 11.1. The 3-cell feed forward network in Figure 21 (3) has associated systems of differential equations defined by a single function $g(u, v)$, where $g$ : $\mathbf{R}^{k} \times \mathbf{R}^{k} \rightarrow \mathbf{R}^{k}$, as follows:

$$
\begin{aligned}
& \dot{x}_{1}=g\left(x_{1}, x_{1}\right) \\
& \dot{x}_{2}=g\left(x_{2}, x_{1}\right) \\
& \dot{x}_{3}=g\left(x_{3}, x_{2}\right) .
\end{aligned}
$$

We can assume that the synchronous equilibrium is at the origin. Let $\alpha=\left(d_{u} g\right)_{0,0}$ be the linearized internal dynamics and let $\beta=\left(d_{v}\right)_{0,0}$ be the linearized coupling. Then

$$
J=\left[\begin{array}{ccc}
\alpha+\beta & 0 & 0 \\
\beta & \alpha & 0 \\
0 & \beta & \alpha
\end{array}\right] .
$$

It follows that the eigenvalues of $J$ are those of the $k \times k$ matrices $\alpha+\beta$ and $\alpha$ with those of $\alpha$ repeated twice. Indeed, when $\alpha$ has critical eigenvalues, generically $J \mid E^{c}$ will be nilpotent.

The architecture of this feed forward network leads to unusual behavior in synchrony-breaking Hopf bifurcation. For simplicity, assume $k=2$, the function $g$ depends on a parameter $\lambda, \alpha+\beta$ has real part negative eigenvalues, and $\alpha$ has purely imaginary eigenvalues at $\lambda=0$. The assumption on $\alpha+\beta$ implies that $x_{1}(t)$ tends to 0 ; without loss of generality we may assume that $x_{1}=0$ and we may 
rewrite (11.1) as

$$
\begin{aligned}
& \dot{x}_{2}=g\left(x_{2}, 0, \lambda\right) \\
& \dot{x}_{3}=g\left(x_{3}, x_{2}, \lambda\right)
\end{aligned}
$$

where the dependence on $\lambda$ is made explicit. The skew-product structure of these equations allows us to solve the second equation using standard Hopf bifurcation techniques and (generically) find a periodic solution in $x_{2}$ whose amplitude growth is the expected $\lambda^{1 / 2}$. As discussed in [32, this solution forces $x_{3}(t)$ to have the same period as $x_{2}(t)$ but to grow at the surprising rate of $\lambda^{1 / 6}$. A simple example is:

$$
g(u, v, \lambda)=\left[\begin{array}{cc}
\lambda & -1 \\
1 & \lambda
\end{array}\right] u-|u|^{2} u-v .
$$

The resulting periodic solution is shown in Figure 28. Note that $\sqrt{\lambda} \approx 0.32$ and $\lambda^{1 / 6} \approx 0.68$. These values are the approximate amplitudes of the oscillations in cells 2 and 3 respectively. However, a single solution cannot establish the relevant power law, so we plot the corresponding bifurcation diagram, containing the above solution, in Figure 29 (Left). To verify the 1/6 power, Figure 29 (Right) plots the square of the amplitude of cell 2 and the sixth power of the amplitude of cell 3 against $\lambda$. The fact that the graphs are almost straight lines verifies the stated power laws.

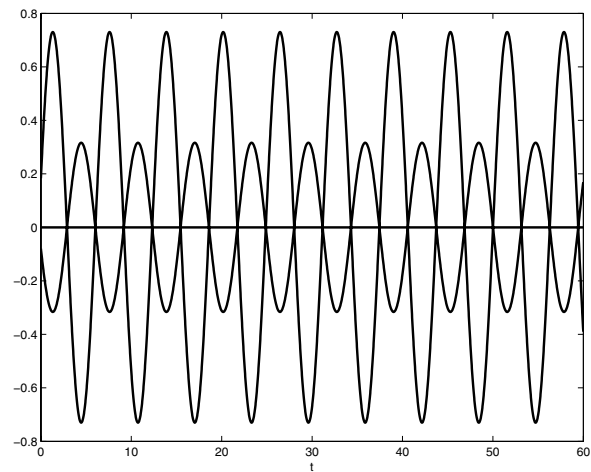

Figure 28. Time series from three-cell network with $g$ as in (11.3) and $\lambda=0.1$. The horizontal line is the time series from cell 1 ; the small amplitude oscillation is from cell 2; and the large amplitude oscillation is from cell 3 .

Example 11.2. One might be tempted to attribute the non-trivial Jordan block to the skew-product structure forced by the feed forward architecture, but the truth must be somewhat subtler [55, 56. Consider network 32 in Figure 22. The admissible systems for this network have the form

$$
\begin{aligned}
& \dot{x}_{1}=g\left(x_{1}, \overline{x_{1}, x_{3}}\right) \\
& \dot{x}_{2}=g\left(x_{2}, \overline{x_{1}, x_{2}}\right) \\
& \dot{x}_{3}=g\left(x_{3}, \overline{x_{1}, x_{2}}\right)
\end{aligned}
$$



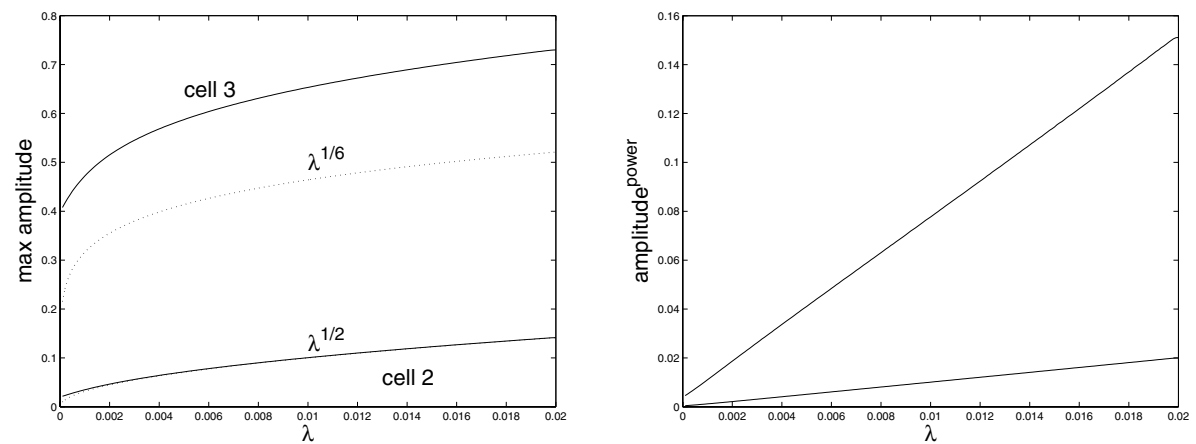

Figure 29. (Left) Maximum amplitude of cells 2 and 3 in (11.3) as a function of $\lambda$. (Right) Square of the amplitude of cell 2 sixth power of the amplitude and cell 3, plotted against $\lambda$.

where $g(u, v, w)=g(u, w, v)$. As before, let $\alpha=\left(d_{u} g\right)_{0,0}$ be the linearized internal dynamics and let $\beta=\left(d_{v}\right)_{0,0}=\left(d_{w} g\right)_{0,0}$ be the linearized coupling. Then

$$
J=\left[\begin{array}{ccc}
\alpha+\beta & 0 & \beta \\
\alpha & \alpha+\beta & 0 \\
\beta & \beta & \alpha
\end{array}\right] .
$$

The eigenvalues of $J$ are again the eigenvalues of $\alpha$ with multiplicity two and the eigenvalues of $\alpha+2 \beta$. The analysis of synchrony-breaking Hopf bifurcation in this network given in 24] shows that generically 2 or 4 branches emanate from this bifurcation (depending on nonlinear terms) and each branch has the standard amplitude growth rate of $\sqrt{\lambda}$.

Example 11.3. The admissible systems of differential equations associated to the homogeneous five-cell network in Figure 30 have the form

$$
\begin{aligned}
& \dot{x}_{1}=g\left(x_{1}, \overline{x_{1}, x_{4}, x_{4}}\right) \\
& \dot{x}_{2}=g\left(x_{2}, \overline{x_{1}, x_{2}, x_{5}}\right) \\
& \dot{x}_{3}=g\left(x_{3}, \overline{x_{2}, x_{4}, x_{4}}\right) \\
& \dot{x}_{4}=g\left(x_{4}, \overline{x_{2}, x_{4}, x_{5}}\right) \\
& \dot{x}_{5}=g\left(x_{5}, \overline{x_{1}, x_{2}, x_{3}}\right)
\end{aligned}
$$

where $x_{j} \in \mathbf{R}^{k}, g: \mathbf{R}^{k} \times \mathbf{R}^{3 k} \rightarrow \mathbf{R}^{k}$, and the overbar indicates that $g(a, b, c, d)$ is invariant under permutation of $b, c, d$. Again we may assume that there is a synchronous equilibrium at the origin.

The Jacobian of (11.5) at the origin has the form

$$
J=\left[\begin{array}{ccccc}
A+B & 0 & 0 & 2 B & 0 \\
B & A+B & 0 & 0 & B \\
0 & B & A & 2 B & 0 \\
0 & B & 0 & A+B & B \\
B & B & B & 0 & A
\end{array}\right]
$$

where $A=g_{1}(0)$ is the linearized internal dynamics and $B=g_{2}(0)=g_{3}(0)=g_{4}(0)$ is the linearized coupling. The $5 k$ eigenvalues of $J$ are given by the $k$ eigenvalues of $A+3 B$ and the $2 k$ eigenvalues of $A \pm i B$ each repeated twice. For example, set $k=1$ and take $A(\lambda)=\lambda$ and $B(\lambda) \equiv-1$. It follows that these coupled cell 


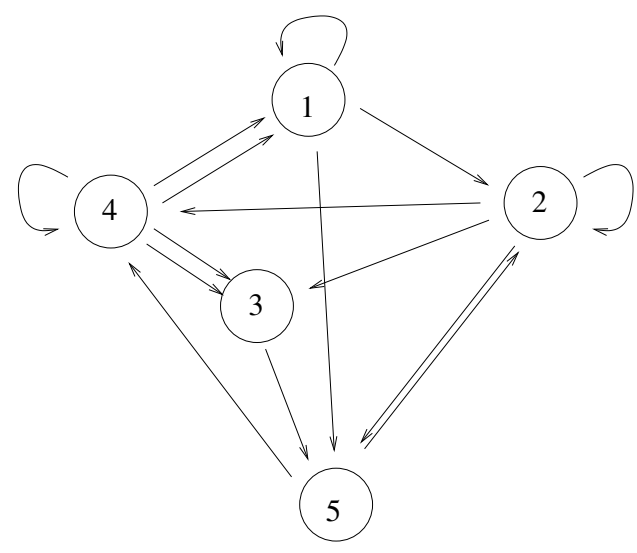

Figure 30. Homogeneous five-cell network that leads to a nilpotent Hopf bifurcation with periodic solutions whose amplitude growth is order $\lambda$.

systems can also undergo a generic nilpotent Hopf bifurcation in codimension one. Generically, this codimension one Hopf bifurcation 24 leads to two branches of periodic solutions with a surprisingly slow growth rate of $\lambda$.

Codimension One Nilpotent Hopf Bifurcations. The standard procedure of Liapunov-Schmidt reduction for finding periodic solutions through Hopf bifurcation (see [34) is still relevant, but nilpotence dramatically changes the analysis. Suppose we look for small amplitude near $2 \pi$-periodic solutions to

$$
\dot{x}=F(x, \lambda),
$$

where $x \in \mathbf{R}^{n}$ and $\lambda \in \mathbf{R}$ under the assumptions that $F(0, \lambda)=0$ and $(d F)_{0,0}$ has a simple pair of purely imaginary eigenvalues $\pm i$ and no other eigenvalues on the imaginary axis. Since the critical eigenvalues are simple it follows that there are smoothly varying eigenvalues $\sigma(\lambda) \pm \omega(\lambda) i$ for $(d F)_{0, \lambda}$.

Since periodic solutions to the nonlinear system will not in general have period $2 \pi$, we can rescale time in the usual way by setting $s=(1+\tau) t$ so that (11.6) becomes

$$
(1+\tau) \frac{d x}{d s}=F(x, \lambda)
$$

and solving for exactly $2 \pi$ periodic solutions to (11.7). Fixing the period in this way allows us to define the operator on loop space $\Phi: \mathcal{C}_{2 \pi}^{1} \times \mathbf{R} \times \mathbf{R} \rightarrow \mathcal{C}_{2 \pi}$ by

$$
\Phi(x, \lambda, \tau)=(1+\tau) \frac{d x}{d s}-F(x, \lambda)
$$

where $\mathcal{C}_{2 \pi}$ and $\mathcal{C}_{2 \pi}^{1}$ are respectively the Banach spaces of continuous and continuously differentiable $2 \pi$-periodic functions on $\mathbf{R}^{n}$. Note that

(a) Solutions to $\Phi(x, \lambda, \tau)=0$ correspond to near $2 \pi$-periodic solutions of (11.6).

(b) $\Phi(0, \lambda, \tau) \equiv 0$ since $F(0, \lambda) \equiv 0$.

(c) $\Phi$ is $\mathbf{S}^{1}$-equivariant, where $\theta \in \mathbf{S}^{1}$ acts on $u \in \mathcal{C}_{2 \pi}$ by

$$
(\theta \cdot u)(s)=u(s-\theta) .
$$


In standard Hopf bifurcation, the dimensions of the kernel and cokernel of the Frechet derivative $(d \Phi)_{0,0,0}$ are 2 and these spaces may be identified with $\mathbf{C}$. Liapunov-Schmidt reduction implies the existence of a mapping $\phi: \mathbf{C} \times \mathbf{R} \times \mathbf{R} \rightarrow \mathbf{C}$ whose zeros near the origin parameterize the small amplitude periodic solutions of $\Phi=0$. Moreover, this reduction can be performed to preserve $\mathbf{S}^{1}$ symmetry; that is, we can assume that

$$
\phi\left(e^{i \theta} z, \lambda, \tau\right)=e^{i \theta} \phi(z, \lambda, \tau)
$$

It follows that

$$
\phi(z, \lambda, \tau)=p\left(|z|^{2}, \lambda, \tau\right) z+q\left(|z|^{2}, \lambda, \tau\right) i z
$$

where $p, q$ are real-valued smooth functions satisfying $p(0,0,0)=q(0,0,0)=0$. Hence solutions to $\phi=0$ are of two types: $z=0$ (the trivial equilibrium) and solutions to the system $p=q=0$ (the desired small-amplitude periodic solutions).

In standard Hopf bifurcation, a calculation shows that $q_{\tau}(0,0,0)=-1$. Hence, the equation $q=0$ can be solved by the Implicit Function Theorem for $\tau=\tau\left(x^{2}, \lambda\right)$, and small amplitude periodic solutions to (11.6) are found by solving

$$
r\left(x^{2}, \lambda\right) \equiv p\left(x^{2}, \lambda, \tau\left(x^{2}, \lambda\right)\right)=0 .
$$

Another (more complicated) calculation shows that

$$
r_{\lambda}(0,0)=\sigma^{\prime}(0) .
$$

It follows from the eigenvalue crossing condition $\sigma^{\prime}(0) \neq 0$ that $r=0$ can be solved by another application of the Implicit Function Theorem for $\lambda=\lambda\left(x^{2}\right)$, and the first Hopf Theorem (existence of a unique branch of small amplitude near $2 \pi$-periodic solutions) is proved. Setting $u=x^{2}$, the second Hopf Theorem (the square root growth in amplitude) is proved by making the genericity assumption $r_{u}(0,0) \neq 0$.

When nilpotence is present in the Jacobian matrix, the eigenvalues are no longer simple, but the kernels and cokernels are still two-dimensional. Therefore, the Liapunov-Schmidt reduction through (11.10) is still valid. However, now

$$
p_{\lambda}(0)=p_{\tau}(0)=q_{\lambda}(0)=q_{\tau}(0)=0
$$

(see 24 for a proof) so simple applications of the Implicit Function Theorem cannot be used to solve $p=q=0$ near the origin.

Solving singular problems in general is a daunting task. However, some structure is forced on these equations by the existence of a nilpotent Hopf bifurcation with critical eigenvalues of algebraic multiplicity two. After a change of coordinates we have 24

$$
p(0, \lambda, \tau)=\lambda^{2}-\tau^{2}+\cdots \quad \text { and } \quad q(0, \lambda, \tau)=-2 \lambda \tau+\cdots .
$$

We now consider the three examples in reverse order. Let $u=|z|^{2}$. If $q_{u}(0) \neq 0$, then $q=0$ can be solved by the Implicit Function Theorem for $u$ and the equation $p(u(\lambda, \tau), \lambda, \tau)=0$ can be solved for two solution branches each with amplitude growth $\lambda$. Generically, this nondegeneracy condition is satisfied for the five-cell network architecture in Example 11.3.

However, network architecture can force $p_{u}(0)=q_{u}(0)=0$. Suppose that generically $q_{u u}(0) \neq 0$. We can write

$$
p(u, \lambda, \tau)=P(u, \lambda, \tau)+\cdots \quad \text { and } \quad q(u, \lambda, \tau)=Q(u, \lambda, \tau)+\cdots
$$


where $P$ and $Q$ are homogeneous quadratic polynomials. In this case, (11.12) implies that there are either two or four solution branches to $P=Q=0$, each with $\lambda^{1 / 2}$ amplitude growth. It can also be shown by a standard blowing-up argument that higher order terms do not matter. This is exactly the situation in Example11.2.

Finally, it was shown in 32 that this three-cell feed-forward network yields two solution branches: one with $\lambda^{1 / 2}$ amplitude growth and one with $\lambda^{1 / 6}$ growth. This observation forces all $u$ derivatives of $p$ and $q$ through order 3 to vanish at the origin. It can be shown 24 that generically $p_{\text {uиuи }}(0) \neq 0$. It follows that there cannot be any other solution branches of small amplitude periodic solutions.

Two General Remarks. In general, preliminary calculations suggest that for every Jordan normal form $J_{0}$ of critical eigenvalues, there is a network with all couplings identical (edge-equivalent) that leads to $J_{0}$ in a codimension-one bifurcation. So it is difficult to conjecture what the analogs of the Equivariant Branching Lemma and the Equivariant Hopf Theorem might be for coupled cell systems.

There is one positive result in this direction. Given a homogeneous coupled cell system with a balanced 2-coloring, there is a codimension one bifurcation from a synchronous equilibrium to a branch of equilibria with that 2-coloring. See [81].

\section{INTERIOR SYMMETRIES}

Group symmetries are global; groupoid symmetries are local. It turns out that there is a useful intermediate concept, a strengthening of input-equivalence 'symmetries' that we call interior symmetry. It was introduced in [33], and in particular it leads to some systematic results on local bifurcation. Like all local bifurcation theories, a key role is played by the eigenvalue structure of linear (admissible) vector fields, and this section motivates the one that follows it on the linear theory. The main aim of this section is to describe analogs of the Equivariant Branching Lemma (41], Chapter XIII, Section 3) and the Equivariant Hopf Theorem [37, 41] in the context of interior symmetries.

Interior symmetries induce extra structure on linearized eigenvalues, and this structure controls the synchrony-breaking local bifurcations.

Definition 12.1. Let $\mathcal{S} \subseteq \mathcal{C}$ be a subset of cells, and let $I(\mathcal{S})=\{i \in \mathcal{E}: \mathcal{H}(i) \in \mathcal{S}\}$. The pair $\left(\sigma, \sigma_{E}\right)$ is an interior symmetry on $\mathcal{S}$ if $\sigma: \mathcal{C} \rightarrow \mathcal{C}$ is an input equivalence preserving permutation that is the identity on the complement of $\mathcal{S}, \sigma_{E}: \mathcal{E} \rightarrow \mathcal{E}$ is an edge equivalence preserving permutation that is the identity on the complement of $I(\mathcal{S})$, and the pair satisfies

$$
\sigma(\mathcal{H}(i))=\mathcal{H}\left(\sigma_{E}(i)\right) \text { and } \sigma(\mathcal{T}(i))=\mathcal{T}\left(\sigma_{E}(i)\right)
$$

for all $i \in I(\mathcal{S})$.

The interior symmetry group $\Sigma_{\mathcal{S}}$ is the set of all interior symmetries on $\mathcal{S}$. It is obviously a group. The interior symmetry group $\Sigma_{\mathcal{C}}$ on the whole network $\mathcal{C}$ is the usual symmetry group $\Gamma$ of the entire coupled cell system. Just like symmetries, interior symmetries force flow-invariant fixed-point subspaces. Suppose that $\mathrm{T} \subset$ $\Sigma_{\mathcal{S}}$ is a subgroup. Then

$$
\operatorname{Fix}(\mathrm{T})=\left\{\left(x_{\mathcal{S}}, x_{\mathcal{C} \backslash \mathcal{S}}\right) \in P: \sigma x_{\mathcal{S}}=x_{\mathcal{S}} \quad \forall \sigma \in \mathrm{T}\right\} .
$$

Note that fixed-point subspaces for groups of interior symmetries are polydiagonals. 
Proposition 12.2. Let $\mathrm{T}$ be a subgroup of $\Sigma_{\mathcal{S}}$, and let $f$ be a $G$-admissible vector field. Then the subspace $\operatorname{Fix}(\mathrm{T})$ is flow-invariant for $f$.

Proof. In the context of interior symmetry, admissibility of the cell system implies that

$$
f_{\mathcal{S}}\left(\sigma x_{\mathcal{S}}, x_{\mathcal{C} \backslash \mathcal{S}}\right)=\sigma f_{\mathcal{S}}\left(x_{\mathcal{S}}, x_{\mathcal{C} \backslash \mathcal{S}}\right)
$$

where $f_{\mathcal{S}}$ is the cell system vector field on the cells in $\mathcal{S}$ and $\sigma \in \Sigma_{\mathcal{S}}$. Observe that if $\left(x_{\mathcal{S}}, x_{\mathcal{C} \backslash \mathcal{S}}\right)$ is in $\operatorname{Fix}(\mathrm{T})$, then it follows from (12.3) that

$$
f_{\mathcal{S}}\left(x_{\mathcal{S}}, x_{\mathcal{C} \backslash \mathcal{S}}\right)=\sigma f_{\mathcal{S}}\left(x_{\mathcal{S}}, x_{\mathcal{C} \backslash \mathcal{S}}\right)
$$

and $f\left(x_{\mathcal{S}}, x_{\mathcal{C} \backslash \mathcal{S}}\right) \in \operatorname{Fix}(T)$.

We now discuss a motivating example:

Example 12.3. The network of Figure 19 is one of the simplest networks with an interior symmetry. Because of the (dotted) arrow from cell 1 to cell 3, the permutation (1 2 2) $\in \mathbf{S}_{3}$ is not a group symmetry of the network, but it is an interior symmetry on the subset $\mathcal{S}=\{1,2\}$.

A state $\left(x_{1}, x_{2}, x_{3}\right)$ is fixed by this interior symmetry if and only if $x_{1}=x_{2}$; that is, cells 1 and 2 are synchronous. That is, they lie in the polydiagonal

$$
\operatorname{Fix}\left(\Sigma_{\mathcal{S}}\right)=\left\{\left(x_{1}, x_{1}, x_{3}\right): x_{1} \in \mathbf{R}^{k}, x_{3} \in \mathbf{R}^{l}\right\}
$$

corresponding to the balanced equivalence relation whose equivalence classes are $\{1,2\},\{3\}$.

Let $x_{0}=\left(x_{1}, x_{1}, x_{3}\right)$ be a synchronous equilibrium for an admissible vector field of the form (5.1). Let $J$ be the Jacobian matrix at $x_{0}$. Then

$$
J=\left[\begin{array}{lll}
a & b & c \\
b & a & c \\
d & 0 & e
\end{array}\right]
$$

where $a, b$ are $k \times k$ matrices, $c$ is a $k \times \ell$ matrix, $d$ is an $\ell \times k$ matrix, and $e$ is an $\ell \times \ell$ matrix. Interior symmetries force a structure on $J$ as follows.

Let $W=\left\{\left(x_{1},-x_{1}, 0\right): x_{1} \in \mathbf{R}^{k}\right\}$; then $W$ is a complementary subspace to $\operatorname{Fix}\left(\Sigma_{\mathcal{S}}\right)$ on which $\Sigma_{\mathcal{S}}$ acts nontrivially $($ as $-I)$. Since $\operatorname{Fix}\left(\Sigma_{\mathcal{S}}\right)$ is flow-invariant, the matrix $J$, written as $L$ in the decomposition $W \oplus \operatorname{Fix}\left(\Sigma_{\mathcal{S}}\right)$, is block lower triangular:

$$
L=\left[\begin{array}{c|cc}
a-b & 0 & 0 \\
\hline 0 & a+b & c \\
d & d & e
\end{array}\right]
$$

Thus synchrony-breaking bifurcations occur when the eigenvalues of $J \mid W$ are critical (that is, the $k \times k$ matrix $a-b$ has eigenvalues with zero real part). In (12.7) and Lemma 12.4 (a) we show that this kind of decomposition occurs generally for linearizations about a synchronous equilibrium supported by an interior symmetry group.

In the context of symmetry-breaking, there are two main local bifurcation theorems [37, 41. The Equivariant Branching Lemma (41], Chapter XIII, Section 3) proves the existence of certain branches of symmetry-breaking steady states; the Equivariant Hopf Theorem (41, Chapter XVI, Section 4) proves the existence of certain branches of spatio-temporal symmetry-breaking time-periodic states. Each 
of these theorems generalizes to synchrony-breaking bifurcations of coupled cell systems with interior symmetry.

The action of the group $\Sigma_{\mathcal{S}}$ decomposes $\mathcal{S}$ as

$$
\mathcal{S}=\mathcal{S}_{1} \cup \cdots \cup \mathcal{S}_{m}
$$

where each $\mathcal{S}_{j}$ is an orbit of the action. Let

$$
W=\left\{x \in P: x_{j}=0 \quad \forall j \in \mathcal{C} \backslash \mathcal{S} \quad \text { and } \quad \sum_{i \in \mathcal{S}_{\ell}} x_{i}=0 \quad \text { for } 1 \leq \ell \leq m\right\} .
$$

Note that $W$ is a $\Sigma_{\mathcal{S}}$-invariant subspace. Moreover, we can write the state space $P$ as

$$
P=W \oplus \operatorname{Fix}\left(\Sigma_{\mathcal{S}}\right) .
$$

In particular, (12.4) implies that vectors in $W$, when written in coupled cell coordinates, have zero components on all cells not in $\mathcal{S}$.

Bifurcation theory concerns changes in solutions of an ODE as parameters are varied, so we introduce an explicit bifurcation parameter $\lambda \in \mathbf{R}$. We assume that $f$ (hence also its components $f_{c}$ ) depend on $\lambda$ and that the ODE

$$
\dot{x}=f(x, \lambda)
$$

has a 'trivial' equilibrium $x_{0} \in \operatorname{Fix}\left(\Sigma_{\mathcal{S}}\right)$. In the present context, we assume that

$$
f\left(x_{0}, \lambda\right) \equiv 0
$$

and that the bifurcation occurs at $\lambda=0$. Let $L=(d f)_{x_{0}}$. Proposition 12.2 implies that $\operatorname{Fix}\left(\Sigma_{\mathcal{S}}\right)$ is invariant under $L$, so that $L$ has the block form

$$
L=\left[\begin{array}{cc}
A & 0 \\
C & B
\end{array}\right]
$$

with respect to the decomposition (12.5); that is, $A: W \rightarrow W$ and $B: \operatorname{Fix}\left(\Sigma_{\mathcal{S}}\right) \rightarrow$ $\operatorname{Fix}\left(\Sigma_{\mathcal{S}}\right)$. Thus the eigenvalues of $L$ are the eigenvalues of $A$, together with those of $B$.

Local bifurcation (steady-state or Hopf) occurs when some eigenvalue of $L$ has zero real part. That eigenvalue is either associated with $A$ or with $B$, and it is the former case that concerns us here. We say that $f$ undergoes a bifurcation at $x_{0}$ that breaks interior symmetry if $A$ has an eigenvalue with zero real part. In this case, steady-state bifurcation occurs when $A$ has a zero eigenvalue, and Hopf bifurcation occurs when $A$ has a conjugate pair of purely imaginary eigenvalues. In the Hopf case, we may assume (after rescaling time if necessary) that the purely imaginary eigenvalues of $A$ are $\pm i$. We assume that the center subspace $E(L)=E(A)$ and that the center subspace $E(A)$ is equal to $\operatorname{ker} A$ in steady-state bifurcation and to the real eigenspace

$$
E(A)=\left\{x \in P:\left(A^{2}+1\right) x=0\right\}
$$

in Hopf bifurcation. The structure of $L$ in (12.7) has several important implications:

Lemma 12.4. (a) A commutes with the action of $\Sigma_{\mathcal{S}}$ on $W$.

(b) If $w \in W$ is an eigenvector of $A$ with eigenvalue $\mu$, then there exists an eigenvector $v$ of $L$ with eigenvalue $\mu$ of the form

$$
v=w+u
$$

where $u \in \operatorname{Fix}\left(\Sigma_{\mathcal{S}}\right)$. 
In particular, Lemma 12.4 (a) can force multiple eigenvalues in synchronybreaking bifurcations. The analog to the Equivariant Branching Lemma for interior symmetries is the following.

Theorem 12.5 ([33]). Assume that $\operatorname{ker} L$ and $\operatorname{ker} A$ have the same dimension. Let $\mathrm{T} \subset \Sigma_{\mathcal{S}}$ be an axial subgroup of the action of $\Sigma_{\mathcal{S}}$ on $\operatorname{ker} A$, and assume the eigenvalue crossing condition. Then there exists a unique branch of equilibria, bifurcating from $x_{0}$, with symmetry group $\mathrm{T}$.

To discuss Hopf bifurcation we require the following concepts. The ODE (12.6) undergoes a synchrony-breaking Hopf bifurcation at $x_{0}$ if, after rescaling time, the linearization $L=(d f)_{x_{0}}$ has eigenvalues $\pm i$ coming from $A$, as defined in (12.7). Since the block matrix $A$ defined in (12.7) does commute with $\Sigma_{\mathcal{S}}$, there is a natural $\Sigma_{\mathcal{S}} \times \mathbf{S}^{1}$-action on $E(A)$, where $\mathbf{S}^{1}$ acts by $\exp (s A)$. Let $\Delta \subset \Sigma_{\mathcal{S}} \times \mathbf{S}^{1}$ be a subgroup. The spatial subgroup of $\Delta$ is $K=\Delta \cap \Sigma_{\mathcal{S}}$.

Definition 12.6. The subgroup $\Delta \subset \Sigma_{\mathcal{S}} \times \mathbf{S}^{1}$ is spatially $\mathbf{C}$-axial if

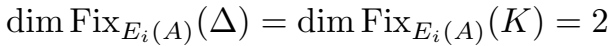

where $K$ is the spatial subgroup of $\Delta$.

The generalization of the Equivariant Hopf Theorem is:

Theorem 12.7 (33]). Assume that a synchrony-breaking Hopf bifurcation occurs at $x_{0}$. Let $\Delta \subset \Sigma_{\mathcal{S}} \times \mathbf{S}^{1}$ be a spatially $\mathbf{C}$-axial subgroup, with spatial subgroup $K$. Then generically there exists a family of periodic solutions of (12.6), bifurcating from $x_{0}$ and having period near $2 \pi$, that is synchronous on any two cells in $\mathcal{S}$ lying in the same $K$-orbit. Moreover, to lowest order in the bifurcation parameter $\lambda$, the solution $x(t)$ has the form

$$
x(t) \approx w(t)+u(t)
$$

where $w(t)=e^{t L} w_{0}$ has exact $\Delta$ spatio-temporal symmetries on cells in $\mathcal{S}$ and $u(t)=e^{t L} u_{0}$ is synchronous on $\Sigma_{\mathcal{S}}$ group orbits of cells in $\mathcal{S}$.

Note that (12.9) follows from Lemma 12.4(b). We illustrate (12.9) by integrating a five-dimensional system of ODE of the form (5.1). The exact system is

$$
\begin{aligned}
g(u, v, w) & =(J+\lambda-1) u-v+w(1,2)^{t}-\left(|u|^{2}+|v|^{2}+w^{2}\right) u \\
h(w, u) & =u_{1}-w-w^{3}
\end{aligned}
$$

where $u, v \in \mathbf{R}^{2}, w \in \mathbf{R}, \lambda=0.05$, and

$$
J=\left[\begin{array}{cc}
0 & -1 \\
1 & 0
\end{array}\right] .
$$

The result is shown in Figure 31 (left). The first coordinates of cells 1 and 2 and cell 3 are shown superimposed in that figure; the largest amplitude signal is from cell 1 and the smallest is from cell 3. To lowest order, the synchronous component $u(t)$ is the average of the time series of cells 1 and 2 . To extract the spatio-temporal symmetries of the component $w(t)$ we therefore subtract that average from the time series for cells 1 and 2. The result is shown in In Figure 31 (right). As predicted by Theorem 12.7 the signals concerned are (approximately) a half-period out of phase. 

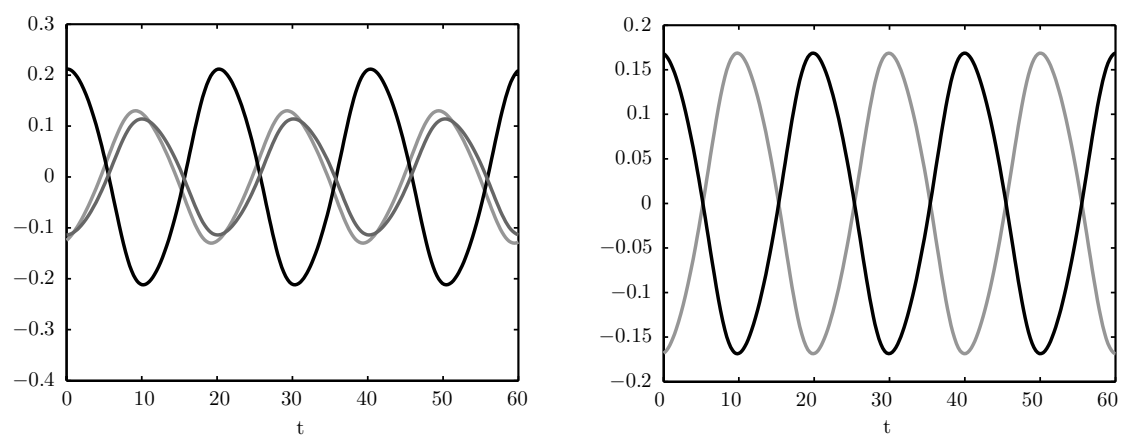

Figure 31. (Left) Simulation from three-cell network in (5.1) with interior $\mathbf{Z}_{2}$ symmetry. (Right) Cells 1 and 2 with average subtracted, illustrating approximate half-period phase shift.

\section{Phase equations}

When modeling time-periodic states of networks, it is common to simplify the dynamics of the cells by assuming them to be phase oscillators. A phase oscillator is a dynamical system whose state space is the circle, so the only variable required to determine the state of a cell is the phase of its oscillation. There is a rigorous reduction from higher-dimensional cell phase spaces to the circle when cells are weakly coupled [49, 52. We follow 31] and apply the theory of coupled cell systems (extended to cell phase spaces that are not vector spaces but manifolds) to such networks, and the results are significant for applications, for instance to 'spiking' in networks of neurons. We give a brief introduction to some of the main ideas, with emphasis on relations among the rotation numbers of cells.

The ODEs for a network of phase oscillators have the form

$$
\dot{\theta}=F(\theta)
$$

where $\theta=\left(\theta_{1}, \ldots, \theta_{N}\right) \in \mathbf{T}^{N}$, and $F=\left(f_{1}, \ldots, f_{N}\right)$. Each coordinate satisfies $0 \leq \theta_{j}<1$, so that the phase space of each oscillator is the unit interval $[0,1]$ with endpoints identified.

Let $F^{L}: \mathbf{R}^{N} \rightarrow \mathbf{R}^{N}$ denote the lift of the vector field $F: \mathbf{T}^{N} \rightarrow \mathbf{R}^{N}$ in (13.1). Suppose that $\theta(t)$ is a solution to (13.1) with initial condition $\theta(0)=\theta_{0}$. The lift of $\theta(t) \in \mathbf{T}^{N}$ to $\mathbf{R}^{N}$ is the solution to $x^{\prime}=F^{L}(x)$ satisfying $x(0)=\theta_{0}$ and is denoted by $\theta^{L}(t)$.

Definition 13.1. Let $\theta(t)$ be a $T$-periodic solution to (13.1). Then the lift satisfies

$$
\theta^{L}(t+T)=\theta^{L}(t)+\rho
$$

where $\rho=\left(\rho_{1}, \ldots, \rho_{N}\right) \in \mathbf{Z}^{N}$ is a vector of integers. The integer $\rho_{j}$ is the rotation number of oscillator $j$.

The rotation number $\rho_{j}$ is simply the number of oscillations of cell $j$ during one period of a periodic solution of (13.1). This notion can be generalized to apply to nonperiodic solutions: 
Definition 13.2. Let $\theta(t)$ be a solution of (13.1) on an interval $\tau=\left[t_{1}, t_{2}\right]$. The oscillation number of $\theta_{j}(t)$ on the interval $\tau$ is given by

$$
\rho_{j}^{\tau}=\theta_{j}^{L}\left(t_{2}\right)-\theta_{j}^{L}\left(t_{1}\right)
$$

Note that $\rho_{j}^{\tau}$ is the number of oscillations of cell $j$ during the time interval $\tau$ and is not, in general, an integer. However, for a $T$-periodic solution and $\tau=[0, T]$, $\rho_{j}^{\tau}$ is an integer and the oscillation number equals the rotation number; that is, $\rho_{j}^{[0, T]}=\rho_{j}$. The oscillation number is closely related to the frequency of oscillation. Given an interval $\tau=\left[t_{1}, t_{2}\right]$, we define the average frequency $\nu_{j}^{\tau}$ over $\tau$ by

$$
\nu_{j}^{\tau}=\frac{\rho_{j}^{\tau}}{t_{2}-t_{1}} .
$$

If the limit

$$
\nu_{i}=\lim _{|\tau| \rightarrow \infty} \nu_{i}^{\tau}
$$

exists, we say that $\nu_{i}$ is the average frequency of cell $j$.

The property of the network that imposes relations between the rotation and oscillation numbers is described in the following definition.

Definition 13.3. Two phase oscillators $i$ and $j$ coevolve if the codimension one torus $\left\{\theta \in \mathbf{T}^{N}: \theta_{i}=\theta_{j}\right\}$ is flow-invariant.

Note that in an all-to-all $\mathbf{S}_{n}$-equivariant coupled system every pair of cells coevolves. The following is proved in [31].

Proposition 13.4. For any periodic solution to a phase oscillator system (13.1), coevolving oscillators have equal rotation numbers. For any solution on an interval $\tau$, all coevolving oscillators have oscillation numbers that differ at most by 1 .

We sketch the reason for these results. Since the torus $\theta_{i}=\theta_{j}$ is assumed to be flow-invariant, the projections $\theta_{i}(t)$ and $\theta_{j}(t)$ of the trajectory $\theta(t)$ are either identical or cannot cross. In other words, oscillators $i$ and $j$ cannot 'lap' or 'pass' one another. Therefore, for a periodic orbit $\theta(t)$, the rotation numbers $\rho_{1}$ and $\rho_{2}$ must be equal. Furthermore, for a general solution on an interval $\tau, \rho_{1}^{\tau}$ and $\rho_{2}^{\tau}$ can differ at most by 1 , since this is the greatest extent to which the phases can be separated without passing.

\section{Synchronized CHAOS}

The topic of synchronized chaos has received a lot of attention in recent years, in part because of possible applications to secure communications. The role of balanced equivalence relations in creating the possibility of synchronous dynamics is not limited to equilibria and periodic states. In particular, the synchronous dynamic can be chaotic. We give an example in which the network is as shown in Figure 32 . which has an interior $\mathbf{Z}_{2}$ symmetry, and the dynamics on the synchrony space is the Rössler attractor (Rössler [69, Peitgen et al. 67]). We also point out some of the subtleties associated with synchronized chaos, in particular the complex behavior associated with instability to synchrony-breaking perturbations, which includes the intriguing phenomenon of 'bubbling'. A bubbling state repeatedly synchronizes and loses synchrony in an intermittent fashion. Even though such a state is only approximately synchronous, it is associated with a flow-invariant subspace. 


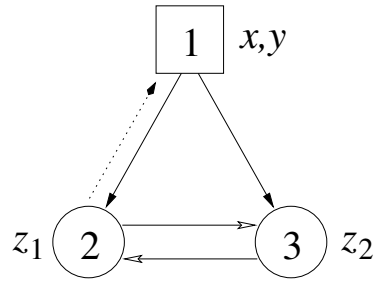

FiguRE 32. Three-cell network with synchronous Rösslerattractor chaos.

Here cell 1 has 2-dimensional phase space with coordinates $(x, y)$, while cells 2 and 3 have 1-dimensional phase spaces with coordinates $z_{1}, z_{2}$ respectively. We give numerical results for the following ODE, which is easily seen to be admissible:

$$
\begin{aligned}
\dot{x} & =-y-z_{1} \\
\dot{y} & =x+a y \\
\dot{z}_{1} & =b+x z_{2}-c z_{1}+k\left(z_{1}-z_{2}\right)+p\left(z_{1}-z_{2}\right)^{2}+q\left(z_{1}-z_{2}\right)^{3} \\
\dot{z}_{2} & =b+x z_{1}-c z_{2}+k\left(z_{2}-z_{1}\right)+p\left(z_{2}-z_{1}\right)^{2}+q\left(z_{2}-z_{1}\right)^{3} .
\end{aligned}
$$

The network has an invariant subspace, namely the synchrony space on which $z_{1}=z_{2}$. Robust synchrony is possible because there is a balanced equivalence relation with classes $\{1\},\{2,3\}$, and associated synchrony space $\Delta=\{x, y, z, z\}$ in which $z_{1}=z_{2}=z$. The dynamic on this subspace is obtained by replacing $z_{1}$ and $z_{2}$ by $z$, which leads to:

$$
\begin{aligned}
\dot{x} & =-y-z \\
\dot{y} & =x+a y \\
\dot{z}_{1} & =b+x z-c z .
\end{aligned}
$$

We recognize this as the standard Rössler system (Rössler [69, Peitgen et al. 67]). Notice that $k, p, q$ do not occur in this system: they affect the dynamics transverse to $\Delta$, but play no role in the dynamics on $\Delta$.

At the chosen parameter values $(a, b, c)$ the system (14.2) has a chaotic attractor $A$, the usual Rössler attractor; see Figure 33. Therefore the system (14.1) has a chaotic invariant set, which we may identify with $A$ using the coordinates $(x, y, z, z)$ on $\Delta$. Now $A$ is an attractor inside $\Delta$, but may or may not be stable to perturbations transverse to $\Delta$. We take up this point in the next section.

In the numerical analysis that follows, we take parameter values $a=0.15, b=$ $0.2, c=5.7, p=-0.001, q=-0.01$, and consider three different values of $k$. The terms in $\left(z_{1}-z_{2}\right)^{3}$ prevent $z_{1}-z_{2}$ blowing up to infinity, and the terms in $\left(z_{1}-z_{2}\right)^{2}$ break the reflectional symmetry $z_{1}-z_{2} \mapsto z_{2}-z_{1}$.

We compare the three cases $k=-2.0,2.9,3.0$. In all cases we choose initial conditions $x(0)=0.1, y(0)=0.12, z_{1}(0)=0.2, z_{2}(0)=0.1$, in which $z_{1}, z_{2}$ are not synchronous. The results of numerical integration in these three cases are shown in Figure 34. The horizontal coordinate is time, and the vertical coordinate is $z_{1}-z_{2}$, which measures to what extent the two cells fail to be synchronous.

The pictures show three distinct kinds of 'synchrony'. When $k=-2.0$ the two cells are identical to within a few parts in $10^{11}$, which strongly suggests the occurrence of an asymptotically stable chaotic attractor lying on the synchrony subspace. When $k=2.9$ the numerics still suggest an asymptotically stable attractor, but we show below that appearances are deceptive. When $k=3.0$ substantial deviations 


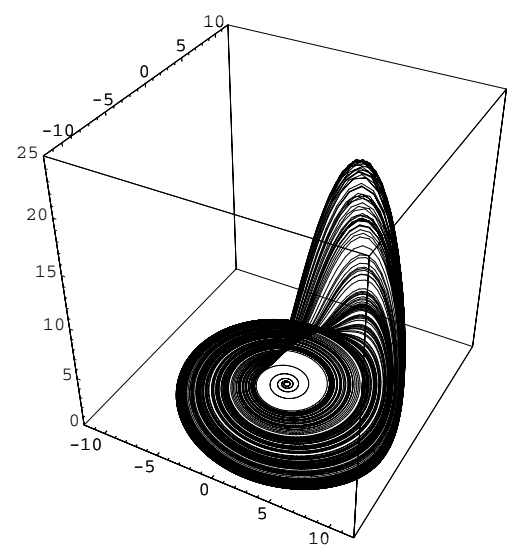

FIGURE 33. Rössler attractor in 3-dimensional synchrony subspace.
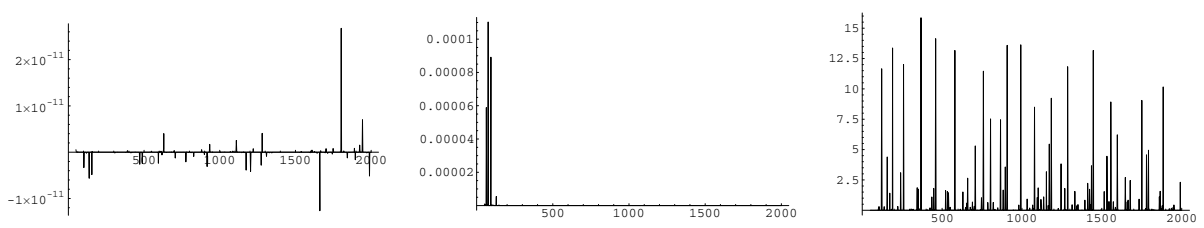

Figure 34. Synchronous Rössler-attractor chaos. Time runs horizontally. Vertical coordinate is $z_{1}-z_{2}$. (Left) $k=-2.0$ : asymptotically stable attractor. Note scale factor $10^{-11}$ on vertical scale. (Middle) $k=2.9$ : Milnor attractor. (Right) $k=3.0$ : bubbling.

from synchrony repeatedly occur: in Section 15 below we explain these in terms of the phenomenon of 'bubbling' (Ashwin et al. [4, [5]).

\section{BubBLing BIFURCATION}

We can also consider the stability of the synchronized chaotic state for the above example. Associated with this is a natural bifurcation scenario: loss of synchrony through a loss of transverse stability. More precisely, although the set $A$ is dynamically invariant and is an attractor inside the synchrony space $\Delta$, it need not be an attractor in the full phase space - the transverse dynamic may cause instability. This context of 'bifurcation from an invariant submanifold' turns out to be surprisingly complex. It is also especially interesting in the network context because of the natural presence of invariant subspaces that may support chaotic dynamics.

The context considered in Ashwin et al. [4, 5] is actually discrete dynamics, that is, iteration of a diffeomorphism $f: M \rightarrow M$ where $M$ is a manifold. We will slide over this distinction.

Suppose that $f$ has an invariant submanifold $N$, so that $\left.f\right|_{N}$ defines a dynamical system on $N$. Suppose further that $\left.f\right|_{N}$ has an (asymptotically stable) attractor $A \subseteq N$. Then $A$ is an invariant subset for $f$. What kind of invariant subset depends on the transverse dynamic - the projected motion orthogonal to $N$ of a point that starts out very close to $A$ but lies outside $N$ ? 
In simple cases this is a classical problem, solved by eigenvalues in the equilibrium case and Floquet multipliers in the periodic case. When $A$ is chaotic, the intuition is that the stability of $A$ in $M$ is determined by the average transverse flow (linearized about $A$ ) with respect to an invariant measure on $A$. However - and this is crucialthere are usually many distinct invariant measures, and these lead to different averages. For example, if $A$ contains a periodic cycle $\Sigma$ of period $\sigma$, then the Dirac measure $\delta_{\Sigma}$ that assigns measure $1 / \sigma$ to each point of $\Sigma$ and is zero everywhere else is invariant. At the other extreme, there may be an SBR (Sinai-Bowen-Ruelle) measure $\mu_{\mathrm{SBR}}$, which is an invariant measure supported on $A$ whose conditional measures on unstable manifolds are absolutely continuous with respect to Lebesgue measure. The average transverse dynamic with respect to $\delta_{\Sigma}$ involves only points in $\Sigma$ and may be expected to differ from the average with respect to $\mu_{\mathrm{SBR}}$.

This difference implies that the associated bifurcation takes place not at a single parameter value but over an interval of parameter values. Transverse instability of $A$ sets in 'gradually' at the start of the interval and is complete by the end of the interval. The most important parameter value lies within the interval and corresponds to a change of sign of the average with respect to the SBR measure. Theorem 15.1 below is a formal statement of what happens.

The transverse dynamic is characterized by a finite set of normal Liapunov exponents, which measure the local rate of expansion or contraction and are analogous to linearized eigenvalues. They depend on a choice of invariant measure $\mu$ and are well-defined provided $\mu$ is ergodic. Define $\lambda_{\min }$ and $\lambda_{\max }$ to be the infimum and supremum of all normal Liapunov exponents over all invariant measures, and let $\lambda_{\mathrm{SBR}}$ be the largest normal Liapunov exponent for the SBR measure. Then

$$
\lambda_{\min } \leq \lambda_{\mathrm{SBR}} \leq \lambda_{\max }
$$

and the signs of these three quantities determine the overall dynamics. Proposition 2.21 of Ashwin et al. [5] summarizes the bifurcation scenario. We restate it as:

Theorem 15.1. Let $f: M \rightarrow M$ be a $C^{1+\alpha}$ map leaving the embedded submanifold $N$ invariant. Let $A$ be an asymptotically stable chaotic attractor for $\left.F\right|_{N}$. Then relative to $f$ acting on $M$, the set $A$ is:

(a) An asymptotically stable attractor if $\lambda_{\max }<0$.

(b) Liapunov unstable if $\lambda_{\max }>0$.

(c) A Milnor attractor if $\lambda_{\mathrm{SBR}}<0<\lambda_{\max }$.

(d) A chaotic saddle if $\lambda_{\min }<0<\lambda_{\mathrm{SBR}}$.

(e) A normally repelling chaotic saddle if $0<\lambda_{\min }$.

Moreover, in case (c) $A$ will have a 'riddled basin' if a suitable technical condition is valid. Here a Milnor attractor (Milnor 58) is an attractor $A$ whose basin of attraction has nonzero Lebesgue measure, and no compact proper subset $A^{\prime} \subseteq A$ has the same basin of attraction as $A$ (up to a set of zero measure). Riddled basins can be thought of as basins with fractal holes; for a rigorous definition see Alexander et al. [2].

In practice, observations of the dynamics in cases (b) will appear to show an asymptotically stable attractor, because the thin set of unstable points nearby will probably not be detected.

Observations in case (c) depend on such factors as the direction of bifurcation (subcritical or supercritical) relative to a given $\mu$. The possibilities include blowout bifurcation (Ott and Sommerer [66]), on-off intermittency (Platt et al. [68]) and 
bubbling (Ashwin et al. [4). Here we focus on bubbling. In this case, the observed orbit repeatedly moves away from $N$ but is then globally reinjected back near $N$. In a sense, this is a kind of homoclinic behavior around the chaotic invariant set $A$.

Computations for Rössler Synchrony. We now relate the above scenario to the system (14.1), which we claim exhibits bubbling. The relevant submanifold $N$ is the synchrony space $\Delta$ on which $z_{1}=z_{2}$. The 'spiking' observed in $z_{1}-z_{2}$ when $k=3.0$ represents repeated (but irregular) loss of synchrony followed by its resumption. This is how bubbling appears when $N$ is a synchrony space.

The dynamics on $\Delta$ has already been derived; see equation (14.2). We analyze the transverse dynamics as follows. Choose the transverse coordinate

$$
u=z_{1}-z_{2}
$$

which vanishes on $\Delta$. From (14.1)

$$
\begin{aligned}
\dot{u} & =\dot{z}_{1}-\dot{z}_{2} \\
& =x\left(z_{2}-z_{1}\right)-c\left(z_{1}-z_{2}\right)+2 k\left(z_{1}-z_{2}\right)+2 p\left(z_{1}-z_{2}\right)^{2}+2 q\left(z_{1}-z_{2}\right)^{3} \\
& =u(-x-c+2 k)+2 p u^{2}+2 q u^{3} .
\end{aligned}
$$

The linearized transverse flow is therefore

$$
\dot{u}=(2 k-c-x) u \text {. }
$$

Since the codimension $m-n=1$, there is a unique normal Liapunov exponent at each point $(x, y, z, z) \in A$. Its value is

$$
\lambda_{k}=2 k-c-x
$$

which depends only on $x$.

Assuming ergodicity of an invariant measure $\mu$, the average linearized flow transverse to $A$, with respect to $\mu$, can be computed as the integral

$$
\int_{A}(2 k-c-x) d \mu=2 k-c-\int_{A} x d \mu .
$$

As a surrogate for the final integral in (15.1) with $\mu=\mu_{\text {SBR }}$ we employ the corresponding ergodic integral

$$
E_{k}=\lim _{T \rightarrow \infty} E_{k}(T) \quad \text { where } \quad E_{k}(T)=\frac{1}{T} \int_{0}^{T}[2 k-c-x(t)] d t .
$$

Numerical computations of $E_{k}$ are shown in the second column of Figure 35, where the horizontal coordinate is $T$ and the vertical one is $E_{k}(T)$.

When $k=-2.0<\lambda_{\min }$, the set $A$ is an asymptotically stable attractor.

As $k$ increases, $E_{k}$ remains negative up to $k=2.9$. The second column shows that $2 k-c-x(t)$ changes sign on $A$ for this parameter value, so $A$ is not an asymptotically stable attractor. Instead, it is a Milnor attractor.

At $k=3.0$, however, $E_{k}>0$, so the SBR-averaged transverse dynamic is repelling, and we observe bubbling. Cells 1 and 2 repeatedly lose synchrony, as shown by the spikes in $z_{1}-z_{2}$, but then return near $\Delta$ and regain synchrony, because trajectories are globally reinjected near $A$.

Coupled cell networks may have robust flow-invariant subspaces, given by balanced equivalence relations. If so, synchronized chaotic attractors may exist on the corresponding synchrony space, provided it has dimension 3 or more. Therefore the 

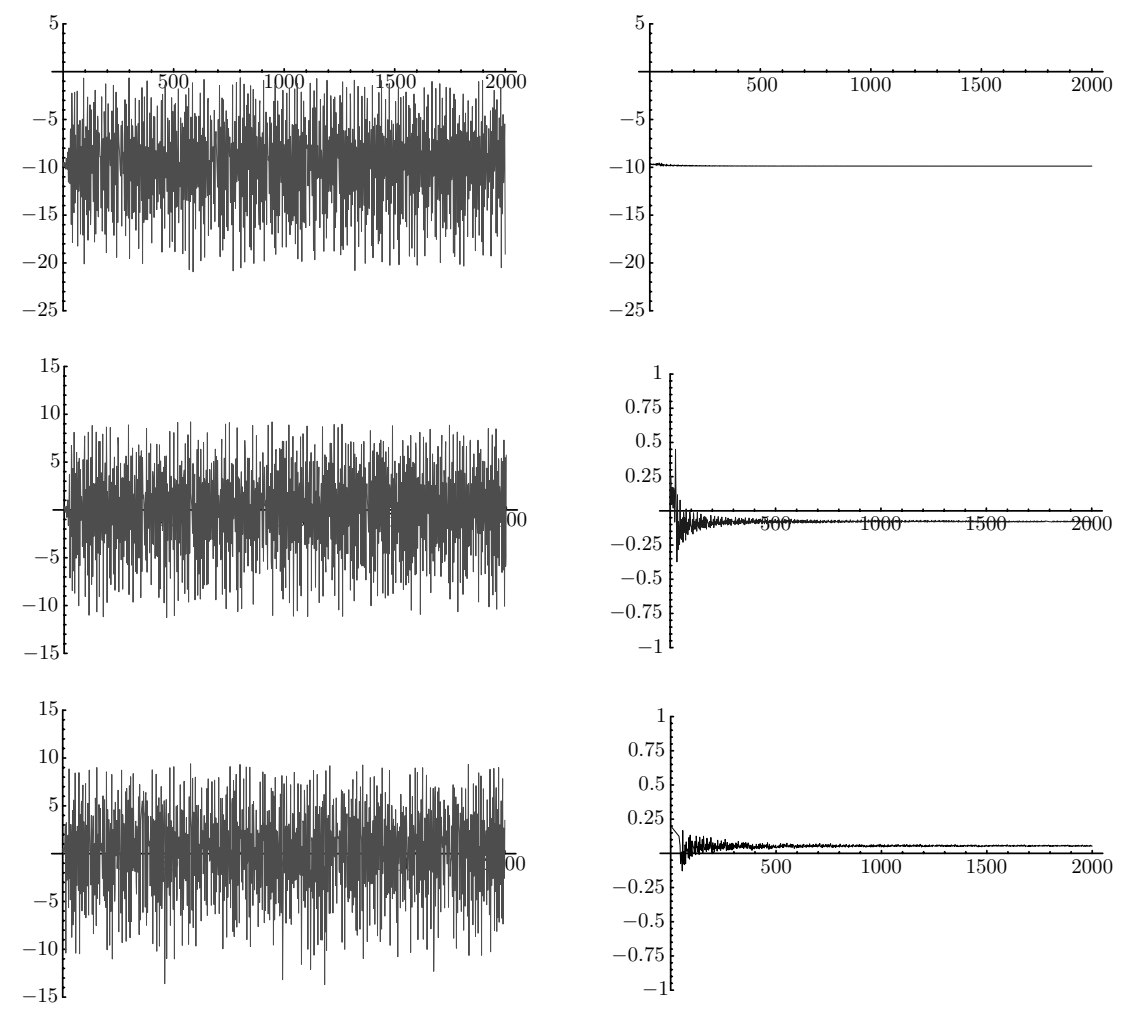

FiguRE 35. Synchronous Rössler-attractor chaos. Time runs horizontally. Vertical coordinate is: (Left) $2 k-c-x$. (Right) Ergodic integral of $2 k-c-x$. (Row 1) $k=-2.0$. Asymptotically stable attractor. (Row 2) $k=2.9$. Milnor attractor. (Row 3) $k=3.0$. Bubbling. Note change of sign (to positive) of ergodic integral.

phenomena associated with the loss of transverse stability of a chaotic attractor lying on an invariant subspace, such as bubbling, are likely to occur. Therefore this kind of intermittent synchronization is likely to be typical in coupled cell systems. So even though our initial definition of 'synchrony' is very strong, the associated concepts of balanced equivalence relations and synchrony subspaces can shed light on more subtle kinds of 'approximate' synchrony.

\section{IS THERE A LINEAR THEORY?}

We end this outline of network dynamics by raising an important, though rather obvious, question. In symmetric dynamics, the presence of a symmetry group has a significant effect on stability properties, and this effect is well understood for equilibria and periodic states. To what extent does something similar hold for the groupoid case?

The natural context here is local bifurcation theory, which employs linear algebra to detect bifurcation points and then analyzes the nature of the resulting states by considering nonlinear terms. The most important local bifurcations are 'codimension one', meaning that they occur generically as a single parameter is varied. The 
principal aims of local bifurcation theory are to obtain criteria for the existence of local branches, to understand their dynamics, and to compute their stabilities.

Consider a general 1-parameter family of ODEs

$$
\dot{x}=f(x, \lambda)
$$

where $x \in \mathbf{R}^{n}, \lambda \in \mathbf{R}$. For simplicity suppose that there is a trivial solution $x(t) \equiv 0$ for all $\lambda$, and let $L(\lambda)=\left.D_{x} f\right|_{(0, \lambda)}$ be the Jacobian at $(0, \lambda)$. Then local bifurcation occurs at values of $\lambda$ for which $L(\lambda)$ has at least one critical eigenvalue $\mu$, that is, an eigenvalue on the imaginary axis.

When $f$ has no special structure (such as symmetry or network constraints), the generic cases are $\mu=0$ and $\mu= \pm i \omega$ for nonzero $\omega \in \mathbf{R}$. Generically, there should be no other critical eigenvalues; in particular, the critical eigenvalues are simple. As $\lambda$ varies through the bifurcation point, generically the critical eigenvalues cross the imaginary axis transversely (that is, with nonzero speed), and this property guarantees the local existence of a bifurcating branch of nonzero states. A zero eigenvalue leads to a branch of steady states, while a conjugate pair of imaginary eigenvalues leads to a branch of periodic solutions.

The stability of the bifurcating branch depends on the eigenvalues of the Jacobian evaluated along the branch. This is a linear algebra problem, but the Jacobian depends on the nonlinear terms because it is evaluated along the branch.

If $f$ has additional structure, what is 'generic' may change. Local bifurcation is still governed by critical eigenvalues, and the real/imaginary distinction still applies, so we can refer to steady-state and Hopf bifurcations. However, the form of $L(\lambda)$ can be more complicated. Symmetry can force multiple eigenvalues; network architecture can do the same, and can also force nonzero nilpotent parts in the appropriate Jordan block.

In the group-symmetric case, there exist satisfactory techniques for analyzing local bifurcations. These techniques are based on the representation theory of the symmetry group $\Gamma$ of $f$. For steady-state bifurcation, a generic zero eigenspace must be an absolutely irreducible component of the representation; for Hopf bifurcation, a generic critical eigenspace is ' $\Gamma$-simple'. Bifurcating branches tend to lie in fixed-point spaces (of subgroups $\Sigma$ of $\Gamma$ in the steady case, and of subgroups $\Sigma$ of the group $\Gamma \times \mathbf{S}^{1}$ of spatio-temporal symmetries in the Hopf case). The Jacobian along the corresponding branch can be block-diagonalized according to the isotypic components of $\Sigma$, which are sums of all irreducible components of a given isomorphism type.

Is there an analogous theory for network dynamics? Some features of the grouptheoretic case carry over. For example, balanced polydiagonals play the same role as fixed-point subspaces. However, it is not so straightforward to find suitable analogs of irreducible representations and isotypic components. If we ask too much of these analogs, they do not exist; if we relax the conditions imposed on them, they may not be very useful.

Even in the group-symmetric case, many aspects of local bifurcation can be very complicated, as the examples of Field [27] demonstrate. It is probably overambitious to expect a complete, general theory of all generic possibilities. So it is not surprising that the groupoid-symmetric case can also be puzzling. Examples (among them many of those discussed in this paper) do reveal a certain degree of structure and suggest that a sensible theory may exist for some types of network, 
even if the general case remains elusive. Of course the linear problem can in principle be solved for any given network architecture; the question is, are there any useful general principles?

A few such results are easily obtained. There is, for instance, a straightforward combinatorial description of the admissible linear maps for a given network, which is an elaboration of its graph-theoretic adjacency matrix. Moreover, the examples discussed above indicate that a certain amount of the eigenvalue structure is common to all linear $G$-admissible matrices $L$ (that is, matrices of linear admissible vector fields). For example, there can exist common invariant subspaces $\Delta$. The obvious examples are polydiagonals corresponding to balanced equivalence relations $\bowtie$. The restriction of $L$ to $\Delta$ corresponds to the quotient network $G / \bowtie$, so some eigenvalues of $L$ are determined by this quotient network. The rest live on the space $P / \Delta$, and the associated matrix induced by $L$ can also be characterized combinatorially.

However, the invariant subspaces determined by balanced equivalence relations are arguably the wrong ones to think about. In the group-theoretic analogy, they correspond to fixed-point subspaces. Such spaces are indeed invariant under all linear equivariant maps. But the most significant spaces of this kind are the isotypic components of the group action, which in turn are determined by the irreducible components. The examples analyzed above show that in the groupoid case there may exist common invariant subspaces for all admissible linear maps which do not correspond to any balanced equivalence relation. Some of these spaces seem to be related to representations of groups (see the interior symmetry section, for example). Others do not.

Can we characterize the generic critical eigenspaces for a given network architecture other than on a case-by case basis? Is there a useful analog of isotypic components in the groupoid case? A serious problem seems to be the absence of any general theorem of 'complete reducibility'. The representation theory is less well-behaved - indeed it is closely related to that of finite-dimensional, possibly nonsemisimple, associative algebras, which is known to be intractable in generaland this presents an obstacle to any general understanding of local bifurcations. We must therefore leave these issues open at the present time.

\section{ACKnowledgments}

We thank our many co-authors on this project: Fernando Antoneli, Luciano Buono, Jim Collins, Ana Dias, Toby Elmhirst, Kreso Josić, Maria Leite, Matt Nicol, Martyn Parker, Marcus Pivato, Eric Shea-Brown, Andrew Török, and Yunjiao Wang. The work of MG was supported in part by NSF Grants DMS-0071735 and DMS-0244529 and ARP Grant 003652-0032-2001. That of IS was supported in part by NSF Grant DMS-0244529 and a grant from EPSRC.

\section{About THE AUTHors}

Martin Golubitsky is Cullen Distinguished Professor of Mathematics at the University of Houston. He is a Fellow of AAAS and the current president of SIAM.

Ian Stewart is a professor at the University of Warwick (UK). He is a recipient of the AAAS Award for the Public Understanding of Science and is a Fellow of the Royal Society. 


\section{REFERENCES}

[1] F. Aldosray and I. Stewart. Enumeration of homogeneous coupled cell networks. Internat. J. Bif. Chaos Appl. Sci. Engrg. MR 2174556

[2] J.C. Alexander, I. Kan, J.A. Yorke, and Zhiping You. Riddled basins, Internat. J. Bif. Chaos 2 (1992) 795-813. MR1206103 (93k:58140)

[3] F. Antoneli, A.P.S. Dias, M. Golubitsky, and Y. Wang. Patterns of synchrony in lattice dynamical systems, Nonlinearity 18 (2005) 2193-2209. MR2164738

[4] P. Ashwin, J. Buescu, and I. Stewart. Bubbling of attractors and synchronisation of oscillators, Phys. Lett. A 193 (1994) 126-139. MR.1295394 (95e:58114)

[5] P. Ashwin, J. Buescu, and I. Stewart. From attractor to chaotic saddle: a tale of transverse instability, Nonlinearity 9 (1996) 703-737. MR1393154 (97k:58096)

[6] P. Ashwin and P. Stork. Permissible symmetries of coupled cell networks, Math. Proc. Camb. Phil. Soc. 116 (1994) 27-36. MR1274157 (95j:92003)

[7] P. Ashwin and J.W. Swift. The dynamics of $n$ identical oscillators with symmetric coupling. J. Nonlin. Sci. 2 (1992) 69-108. MR1158354 (93g:58103)

[8] N.L. Biggs. Discrete Mathematics, Oxford University Press, Oxford, $1989 . \quad$ MR.1078626 (91h:00002)

[9] J. Blaszczyk and C. Dobrzecka. Alteration in the pattern of locomotion following a partial movement restraint in puppies, Acta. Neuro. Exp. 49 (1989) 39-46.

[10] S. Boccaletti, L.M. Pecora, and A. Pelaez. A unifying framework for synchronization of coupled dynamical systems, Phys. Rev. E 63 (2001) 066219.

[11] H. Brandt. Über eine Verallgemeinerung des Gruppenbegriffes, Math. Ann. 96 (1927) 360366. MR1512323

[12] R. Brown. From groups to groupoids: a brief survey, Bull. London Math. Soc. 19 (1987) 113-134.

[13] P.L. Buono and M. Golubitsky. Models of central pattern generators for quadruped locomotion: I. primary gaits. J. Math. Biol. 42, No. 4 (2001) 291-326. MR1834105 (2002f:92003)

[14] R. Calabrese and E. Peterson. Neural control of heartbeat in the leech, Hirudo medicinalis, in Neural Origin of Rhythmic Movements (A. Roberts and B. Roberts, eds.), Symp. Soc. Exp. Biol. 37 (1983) 195-221.

[15] J. Cohen and I. Stewart. Polymorphism viewed as phenotypic symmetry-breaking, in: Nonlinear Phenomena in Physical and Biological Sciences (S.K. Malik, ed.), Indian National Science Academy, New Delhi, 1-67.

[16] J.J. Collins and I. Stewart. Hexapodal gaits and coupled nonlinear oscillator models, Biol. Cybern. 68 (1993) 287-298.

[17] J.J. Collins and I. Stewart. Coupled nonlinear oscillators and the symmetries of animal gaits, J. Nonlin. Sci. 3 (1993) 349-392. MR1237096 (94g:92007)

[18] A.P.S. Dias and I. Stewart. Symmetry groupoids and admissible vector fields for coupled cell networks, J. London Math. Soc. 69 (2004) 707-736. MR2050042 (2005j:37034)

[19] A.P.S. Dias and I. Stewart. Linear equivalence and ODE-equivalence for coupled cell networks, Nonlinearity 18 (2005) 1003-1020. MR2134081 (2006e:37029)

[20] B. Dionne, M. Golubitsky, and I. Stewart. Coupled cells with internal symmetry, Part 1: wreath products, Nonlinearity 9 (1996) 559-574. MR.1384492 (97j:58110)

[21] B. Dionne, M. Golubitsky, and I. Stewart. Coupled cells with internal symmetry, Part 2: direct products, Nonlinearity 9 (1996) 575-599. MR1384493 (97j:58111)

[22] R. Dobrin, Q.K. Beg, A.-L. Barabási, and Z.N. Oltvai. Aggregation of topological motifs in the Escherichia coli transcriptional regulatory network, BMC Bioinformatics 5 (2004) $1471-2105 / 5 / 10$.

[23] T. Elmhirst. Symmetry and Emergence in Polymorphism and Sympatric Speciation, Ph.D. Thesis, Math. Inst., U. Warwick, 2002.

[24] T. Elmhirst and M. Golubitsky. Nilpotent Hopf bifurcations in coupled cell systems, SIAM J. Appl. Dynam. Sys. 5 (2006). To appear.

[25] I.R. Epstein and M. Golubitsky. Symmetric patterns in linear arrays of coupled cells, Chaos 3(1) (1993) 1-5. MR.1210158 (94a:80015)

[26] M. Feinberg. The existence and uniqueness of steady states for a class of chemical reaction networks, Arch. Rational Mech. Anal. 132 (1995) 311-370. MR.1365832 (97g:92028) 
[27] M.J. Field. Lectures on Bifurcations, Dynamics and Symmetry, Research Notes in Mathematics 356, Pitman, San Francisco, 1996. MR.1425388 (97h:58115)

[28] R. FitzHugh. Impulses and physiological states in theoretical models of nerve membrane, Biophys. J. 1 (1961) 445-466.

[29] P.P. Gambaryan. How Mammals Run: Anatomical Adaptations, Wiley, New York, 1974.

[30] D. Gillis and M. Golubitsky. Patterns in square arrays of coupled cells, J. Math. Anal. App. 208 (1997) 487-509. MR1441450 (98g:58158)

[31] M. Golubitsky, K. Josić, and E. Shea-Brown. Rotation, oscillation and spike numbers in phase oscillator networks, J. Nonlinear Sci. To appear.

[32] M. Golubitsky, M. Nicol, and I. Stewart. Some curious phenomena in coupled cell networks, J. Nonlinear Sci. 14 (2004) 119-236. MR2041431 (2005a:37037)

[33] M. Golubitsky, M. Pivato, and I. Stewart. Interior symmetry and local bifurcation in coupled cell networks, Dyn. Sys. 19 (2004) 389-407 MR2107649

[34] M. Golubitsky and D.G. Schaeffer. Singularities and Groups in Bifurcation Theory: Vol. I, Applied Mathematical Sciences 51, Springer-Verlag, New York, 1985. MR0771477 (86e:58014)

[35] M. Golubitsky and I. Stewart. Hopf bifurcation with dihedral group symmetry: coupled nonlinear oscillators, in Multiparameter Bifurcation Theory (M. Golubitsky and J. Guckenheimer, eds.), Proceedings of the AMS-IMS-SIAM Joint Summer Research Conference, July 1985, Arcata; Contemporary Math. 56, Amer. Math. Soc., Providence, RI, 1986, 131-173. MR0855088 (88c:58047)

[36] M. Golubitsky and I. Stewart. Symmetry and pattern formation in coupled cell networks, In: Pattern Formation in Continuous and Coupled Systems (M. Golubitsky, D. Luss, and S.H. Strogatz, eds.), IMA volumes in Math. and Appls. 115, Springer-Verlag, New York, 1999, 65-82. MR.1708862 (2001h:34060)

[37] M. Golubitsky and I. Stewart. The Symmetry Perspective: From Equilibrium to Chaos in Phase Space and Physical Space, Progress in Mathematics 200, Birkhäuser, Basel, 2002. MR1891106 (2003e:37068)

[38] M. Golubitsky, I. Stewart, P.-L. Buono, and J.J. Collins. A modular network for legged locomotion, Physica D 115 (1998) 56-72. MR1616780(99d:92051)

[39] M. Golubitsky, I. Stewart, P.-L. Buono, and J.J. Collins. Symmetry in locomotor central pattern generators and animal gaits, Nature 401 (1999) 693-695.

[40] M. Golubitsky, I. Stewart, and B. Dionne. Coupled cells: wreath products and direct products, in Dynamics, Bifurcation and Symmetry, NATO ARW Series (P. Chossat and J.M. Gambaudo, eds.), Kluwer, Amsterdam, 1994, 127-138. MR.1305373 (96e:58115)

[41] M. Golubitsky, I. Stewart, and D.G. Schaeffer. Singularities and Groups in Bifurcation Theory II, Applied Mathematics Sciences, 69, Springer-Verlag, New York, 1988. MR0950168 (89m:58038)

[42] M. Golubitsky, I. Stewart, and A. Török. Patterns of synchrony in coupled cell networks with multiple arrows, SIAM J. Appl. Dyn. Sys. 4(1) (2005) 78-100. MR2136519 (2005k:34143)

[43] M.G.M. Gomes and G.F. Medley. Dynamics of multiple strains of infectious agents coupled by cross-immunity: a comparison of models, in Mathematical Approaches for Emerging and Reemerging Infectious Diseases (S. Blower, C. Castillo-Chavez, K.L. Cooke, D. Kirschner, and P. van der Driessche, eds.), Springer-Verlag, New York, 2002, 171-191. MR1938903

[44] S. Grillner, D. Parker, and A.J. El Manira. Vertebrate locomotion-a lamprey perspective, Ann. New York Acad. Sci. 860 (1998) 1-18.

[45] S. Grillner and P. Wallén. Central pattern generators for locomotion, with special reference to vertebrates, Ann. Rev. Neurosci 8 (1985) 233-261.

[46] B. Gucciardi. Thesis, University of Houston, 2006. In preparation.

[47] P.J. Higgins. Notes on Categories and Groupoids, Van Nostrand Reinhold Mathematical Studies 32, Van Nostrand Reinhold, London, 1971. MR0327946 (48:6288)

[48] M.W. Hirsch, C.C. Pugh, and M. Shub. Invariant Manifolds, Lect. Notes Math. 583, Springer, New York, 1977. MR0501173 (58:18595)

[49] F.C. Hoppensteadt and I. Izhekevich. Weakly Connected Neural Nets, Applied Mathematical Sciences 126, Springer, New York, 1997. MR 1458890 (98k:92004)

[50] A. Katok and B. Hasselblatt. Introduction to the Modern Theory of Dynamical Systems, Cambridge U. Press, Cambridge, 1995. MR1326374 (96c:58055) 
[51] N. Kopell and G.B. Ermentrout. Symmetry and phaselocking in chains of weakly coupled oscillators, Comm. Pure Appl. Math. 39 (1986) 623-660. MR0849426 (87m:34054)

[52] N. Kopell and G.B. Ermentrout. Coupled oscillators and the design of central pattern generators, Math. Biosci. 90 (1988) 87-109. MR0958133 (89m:92024)

[53] N. Kopell and G. LeMasson. Rhythmogenesis, amplitude modulation, and multiplexing in a cortical architecture, Proc. Natl. Acad. Sci. USA 91 (1994) 10586-10590.

[54] Y. Kuramoto. Chemical Oscillations, Waves, and Turbulence, Springer, Berlin, 1984. MR0762432 (87e:92054)

[55] M. Leite. Homogeneous three-cell networks. Ph.D. Thesis, University of Houston, August 2005. MR:1697351(2000f:37111)

[56] M. Leite and M. Golubitsky. Homogeneous three-cell networks. Nonlinearity. Submitted.

[57] S.C. Manrubia, A.S. Mikhailov, and D.H. Zanette, Emergence of Dynamical Order, World Scientific, Singapore, 2004.

[58] J. Milnor. On the concept of attractor, Commun. Math. Phys. 99 (1985) 177-195. MR0790735 (87i:58109a)

[59] R. Milo, S. Shen-Orr, S. Itkovitz, N. Kashtan, D. Chklovskii, and U. Alon. Network motifs: simple building blocks of complex networks, Science 298 (2002) 824.

[60] E. Mosekilde, Y. Maistrenko, and D. Postonov, Chaotic Synchronization, World Scientific, Singapore, 2002. MR.1939912 (2004h:37045)

[61] J.S. Nagumo, S. Arimoto, and S. Yoshizawa. An active pulse transmission line simulating nerve axon, Proc. IRE 50 (1962) 2061-2071.

[62] A.-M. Neutel, J.A.P. Heesterbeek, and P.C. de Ruiter. Stability in real food webs: weak links in long loops, Science 96 (2002) 1120-1123.

[63] M.E.J. Newman. The structure and function of complex networks, SIAM Review 45 (2003) 167-256. MR2010377 (2005a:05206)

[64] O.H. Olsen and R.L. Calabrese. Activation of intrinsic and synaptic currents in leech heart interneurons by realistic waveforms, J. Neuroscience 16 4958-4970.

[65] Z.N. Oltvai and A.-L. Barabási. Life's complexity pyramid, Science 298 (2002) 763-764.

[66] E. Ott and J.C. Sommerer. Blowout bifurcations: the occurrence of riddled basins and on-off intermittency, Phys. Lett. A 188 (1994) 39-47.

[67] H.-O. Peitgen, H. Jürgens, and D. Saupe. Chaos and Fractals, Springer-Verlag, New York, 1992. MR.1185709 (93k:58157)

[68] N. Platt, E.A. Spiegel, and C. Tresser. On-off intermittency: a mechanism for bursting, Phys. Rev. Lett. 70 (1993) 279-282.

[69] O.E. Rössler. An equation for continuous chaos, Phys. Lett. 57A (1976) 397-398.

[70] W. Singer. Neuronal synchrony: a versatile code for the definition of relations, Neuron 24 (1999) 49-65.

[71] I. Stewart, T. Elmhirst, and J. Cohen. Symmetry-breaking as an origin of species. In: Bifurcation, Symmetry and Patterns (J. Buescu, S.B.S.D. Castro, A.P.S. Dias, and I.S. Labouriau, eds.), Birkhäuser, Basel, 2003, 3-54. MR2014354 (2005a:92032)

[72] I. Stewart, M. Golubitsky, and M. Pivato. Patterns of synchrony in coupled cell networks, SIAM J. Appl. Dynam. Sys. 2 (2003) 609-646. [DOI: 10.1137/S1111111103419896] MR2050244 (2005i:37030)

[73] I. Stewart and M. Parker. Periodic dynamics of coupled cell networks I: rigid patterns of synchrony. Preprint.

[74] I. Stewart and M. Parker. Periodic dynamics of coupled cell networks II: cyclic symmetry. Preprint.

[75] I. Stewart and M. Parker. Periodic dynamics of coupled cell networks III: rigid phase patterns. Preprint.

[76] W.T. Tutte. Graph Theory, Encyclopedia of Mathematics and Its Applications (G.-C. Rota, ed.), 21, Addison-Wesley, Reading, MA, 1984. MR0746795 (87c:05001)

[77] J.J. Tyson, K.C. Chen, and B. Novak. Sniffers, buzzers, toggles and blinkers: dynamics of regulatory and signaling pathways in the cell, Curr. Opin. Cell Biol. 15 (2003) 221-231.

[78] J.J. Tyson, A. Csikasz-Nagy, and B. Novak. The dynamics of cell cycle regulation, BioEssays 24 (2002) 1095-1109.

[79] T.L. Vincent and T.L.S. Vincent. Evolution and control system design, IEEE Control Systems Magazine (October 2000) 20-35. 
[80] X.-F. Wang. Complex networks: Topology, dynamics and synchronization, International Journal of Bifurcation and Chaos 12 (2002) 885-916. MR.1913980

[81] Y. Wang and M. Golubitsky. Two-colour patterns of synchrony in lattice dynamical systems, Nonlinearity 18 (2005) 631-657. MR2122678 (2005i:37032)

[82] D.J. Watts and S.H. Strogatz. Collective dynamics of small-world networks, Nature 393 440-442.

[83] A. Weinstein. Groupoids: unifying internal and external symmetry, Notices Amer. Math. Soc. 43 (1996) 744-752. MR1394388 (97f:20072)

[84] R.J. Wilson. Introduction to Graph Theory (3rd ed.), Longman, Harlow, 1985. MR0826772 (87a:05051)

[85] D.M. Wolf and A.P. Arkin. Motifs, modules, and games in bacteria, Current Opinion in Microbiol. 6 (2003) 125-134.

[86] D. Wood. Coupled Oscillators with Internal Symmetries, Ph.D. Thesis, Univ. Warwick, 1995.

[87] D. Wood. Hopf bifurcations in three coupled oscillators with internal $\mathbf{Z}_{2}$ symmetries, Dyn. Stab. Sys. 13 (1998) 55-93. MR1624208(99e:58136)

[88] D. Wood. A cautionary tale of coupling cells with internal symmetries, Internat. J. Bif. Chaos 11 (2001) 123-132. MR1815530(2001m:37106)

[89] C.W. Wu. Synchronization in Coupled Chaotic Circuits and Systems, World Scientific, Singapore, 2002. MR.1891843 (2003g:34106)

[90] C.W. Wu. Synchronization in networks of nonlinear dynamical systems coupled via a directed graph, Nonlinearity 18 (2005) 1057-1064. MR2134084(2005m:37059)

Department of Mathematics, University of Houston, Houston, Texas 77204-3008

Mathematics Institute, University of Warwick, Coventry CV4 7AL, UK 